Publ. RIMS Kyoto Univ. 47 (2011), 99,140

DOI $10.2977 /$ PRIMS/32

\title{
dg-Methods for Microlocalization
}

\author{
Dedicated to Professor Mikio Sato on the occasion of his 80th birthday \\ by \\ Stéphane Guillermou
}

\begin{abstract}
For a complex manifold $X$ the ring $\mathcal{E}_{X}$ of microdifferential operators acts on the microlocalization $\mu h o m\left(F, \mathcal{O}_{X}\right)$ for $F$ in the derived category of sheaves on $X$. Kashiwara, Schapira, Ivorra and Waschkies proved, as a byproduct of their new microlocalization functor for ind-sheaves, $\mu_{X}$, that $\mu h o m\left(F, \mathcal{O}_{X}\right)$ can in fact be defined as an object of $\mathrm{D}\left(\mathcal{E}_{X}\right)$ : this follows from the fact that $\mu_{X} \mathcal{O}_{X}$ is concentrated in one degree.

In this paper we prove that the tempered microlocalization $T$ - $\mu h o m\left(F, \mathcal{O}_{X}\right)$ and in fact $\mu_{X} \mathcal{O}_{X}^{t}$ are also objects of $\mathrm{D}\left(\mathcal{E}_{X}\right)$. Since we do not know whether $\mu_{X} \mathcal{O}_{X}^{t}$ is concentrated in one degree we build resolutions of $\mathcal{E}_{X}$ and $\mu_{X} \mathcal{O}_{X}^{t}$ such that the action of $\mathcal{E}_{X}$ is realized in the category of complexes (and not only up to homotopy). To define these resolutions we introduce a version of the de Rham algebra on the subanalytic site which is quasi-injective. We prove that some standard operations in the derived category of sheaves can be lifted to the (non-derived) category of dg-modules over this de Rham algebra. Then we build the microlocalization in this framework.
\end{abstract}

2010 Mathematics Subject Classification: 35A27, 32C38.

Keywords: D-modules, microdifferential operators, microlocalization.

\section{§1. Introduction}

For a complex analytic manifold the sheaf of microdifferential operators on its cotangent bundle was introduced in [12] by Sato, Kawai and Kashiwara using Sato's microlocalization functor. Let us recall briefly the definition, in the framework of [5]. Let $X$ be a real manifold and let $\mathrm{D}^{b}\left(\mathbf{C}_{X}\right)$ be the bounded derived category of sheaves of $\mathbf{C}$-vector spaces on $X$. For objects $F, G \in \mathrm{D}^{b}\left(\mathbf{C}_{X}\right)$ a gener-

This is a contribution to the special issue "The golden jubilee of algebraic analysis".

Communicated by M. Kashiwara. Received March 23, 2009.

S. Guillermou: Université de Grenoble I, Département de Mathématiques, Institut Fourier, UMR 5582 du CNRS, 38402 Saint-Martin d'Hères Cedex, France;

e-mail: Stephane.Guillermou@ujf-grenoble.fr

web: ww-fourier.ujf-grenoble.fr/ guillerm

(C) 2011 Research Institute for Mathematical Sciences, Kyoto University. All rights reserved. 
alization of Sato's microlocalization functor gives $\mu h o m(F, G) \in \mathrm{D}^{b}\left(\mathbf{C}_{T^{*} X}\right)$ and a convolution product is defined in [5] for this functor $\mu$ hom. When $X$ is a complex analytic manifold of complex dimension $d_{X}$ one version of the ring of microdifferential operators is $\mathcal{E}_{X}^{\mathbf{R}}=\mu h o m\left(\mathbf{C}_{\Delta}, \mathcal{O}_{X \times X}^{\left(0, d_{X}\right)}\right)\left[d_{X}\right]$, where $\Delta$ is the diagonal of $X \times X$ and $\mathcal{O}_{X \times X}^{\left(0, d_{X}\right)}$ denotes the holomorphic forms of degree 0 on the first factor and degree $d_{X}$ on the second factor. Its support is the conormal bundle of $\Delta$, identified with $T^{*} X$. The product in $\mathcal{E}_{X}^{\mathrm{R}}$ is given by the convolution product in $\mu$ hom.

The convolution product also induces an action of $\mathcal{E}_{X}^{\mathbf{R}}$ on $\mu h o m\left(F, \mathcal{O}_{X}\right)$ for any $F \in \mathrm{D}^{b}\left(\mathbf{C}_{X}\right)$, i.e. a morphism in $\mathrm{D}^{b}\left(\mathbf{C}_{T^{*} X}\right), \mathcal{E}_{X}^{\mathbf{R}} \otimes \mu h o m\left(F, \mathcal{O}_{X}\right) \rightarrow \mu h o m\left(F, \mathcal{O}_{X}\right)$, satisfying the condition of Definition 3.1 below.

A natural question is then whether $\mu h o m\left(F, \mathcal{O}_{X}\right)$ has a natural construction as an object of $\mathrm{D}^{b}\left(\mathcal{E}_{X}^{\mathbf{R}}\right)$. It was answered positively in [9] as a byproduct of the construction of a microlocalization functor for ind-sheaves. The category of indsheaves on $X, \mathbf{I}\left(\mathbf{C}_{X}\right)$, is introduced and studied in 7 . It comes equipped with an internal Hom functor, $\mathcal{I H}$ om, and contains $\operatorname{Mod}\left(\mathbf{C}_{X}\right)$ as a full subcategory; the embedding of $\operatorname{Mod}\left(\mathbf{C}_{X}\right)$ in $\mathbf{I}\left(\mathbf{C}_{X}\right)$ admits a left adjoint (which corresponds to taking the limit) $\alpha_{X}: \mathbf{I}\left(\mathbf{C}_{X}\right) \rightarrow \operatorname{Mod}\left(\mathbf{C}_{X}\right)$, which is exact. In this framework the construction of 9 yields a new microlocalization functor $\mu_{X}: \mathrm{D}^{b}\left(\mathbf{I}\left(\mathbf{C}_{X}\right)\right) \rightarrow$ $\mathrm{D}^{b}\left(\mathbf{I}\left(\mathbf{C}_{T^{*} X}\right)\right)$ such that

$$
\mu h o m(F, G) \simeq \alpha_{T^{*} X} \mathrm{R} \mathcal{I} \mathcal{H o m}\left(\mu_{X} F, \mu_{X} G\right) .
$$

In particular $\mu h o m(F, G)$ takes the form of the usual Hom functor between objects on $T^{*} X$

The convolution product is also defined in this context and now it gives an action of $\mathcal{E}_{X}^{\mathbf{R}}$ on $\mu_{X}\left(\mathcal{O}_{X}\right)$. Through isomorphism (1) this action on $\mu_{X}\left(\mathcal{O}_{X}\right)$ induces an action on $\mu h o m\left(F, \mathcal{O}_{X}\right)$. Hence it is enough to define $\mu_{X}\left(\mathcal{O}_{X}\right)$ as an object of $\mathrm{D}^{b}\left(\mathcal{E}_{X}^{\mathbf{R}}\right)$ to have the answer for all $\mu h o m\left(F, \mathcal{O}_{X}\right)$. It turns out that, outside the zero section of $T^{*} X, \mu_{X}\left(\mathcal{O}_{X}\right)$ is concentrated in degree $-d_{X}$. Thus $\mu_{X}\left(\mathcal{O}_{X}\right) \simeq$ $H^{-d_{X}} \mu_{X}\left(\mathcal{O}_{X}\right)\left[d_{X}\right]$ and, since the action of $\mathcal{E}_{X}^{\mathbf{R}}$ gives an $\mathcal{E}_{X}^{\mathbf{R}}$-module structure on $H^{-d_{X}} \mu_{X}\left(\mathcal{O}_{X}\right)$, we see that $\mu_{X}\left(\mathcal{O}_{X}\right)$ naturally belongs to $\mathrm{D}^{b}\left(\mathcal{E}_{X}^{\mathbf{R}}\right)$.

However in many situations differential operators of finite order are more appropriate. In this paper we solve the same problem in the tempered situation. The tempered microlocalization $T$ - $\mu$ hom $\left(F, \mathcal{O}_{X}\right)$ is introduced in [1] and also has a reformulation in terms of ind-sheaves. Namely it makes sense to consider the indsheaf of tempered $\mathcal{C}^{\infty}$-functions and the corresponding Dolbeault complex $\mathcal{O}_{X}^{t}$ (it is actually a motivation for the theory of ind-sheaves). Then

$$
T-\mu h o m\left(F, \mathcal{O}_{X}\right) \simeq \alpha_{T^{*} X} \mathrm{R} \mathcal{I} \mathcal{H} \operatorname{om}\left(\mu_{X} F, \mu_{X} \mathcal{O}_{X}^{t}\right) .
$$


Replacing $\mu h o m$ by $T$ - $\mu$ hom in the above definition of $\mathcal{E}_{X}^{\mathbf{R}}$ yields another sheaf of microdifferential operators, $\mathcal{E}_{X}^{\mathbf{R}, f}$. We have as above a natural action of $\mathcal{E}_{X}^{\mathbf{R}, f}$ on $\mu_{X}\left(\mathcal{O}_{X}^{t}\right)$. Unfortunately this last complex is a priori not concentrated in one degree and we cannot conclude directly that $\mu_{X}\left(\mathcal{O}_{X}^{t}\right)$ is an object of $\mathrm{D}^{b}\left(\mathcal{E}_{X}^{\mathbf{R}, f}\right)$.

We will in fact find resolutions of $\mathcal{E}_{X}^{\mathbf{R}, f}$ and $\mu_{X}\left(\mathcal{O}_{X}^{t}\right)$ such that the action corresponds to a dg-module structure over a dg-algebra. More precisely we will define an ind-sheaf of dg-algebras $\mathcal{E}_{X}^{\mathcal{A}}$ on $T^{*} X$ (outside the zero section) with cohomology only in degree 0 and such that $H^{0}\left(\mathcal{E}_{X}^{\mathcal{A}}\right)=\mathcal{E}_{X}^{\mathbf{R}, f}$. We will also find a $\operatorname{dg}_{-} \mathcal{E}_{X}^{\mathcal{A}}$-module, say $M$, such that $M \simeq \mu_{X}\left(\mathcal{O}_{X}^{t}\right)$ in $\mathrm{D}^{b}\left(\mathbf{I}\left(\mathbf{C}_{T^{*} X}\right)\right)$ and such that the morphism of complexes $\mathcal{E}_{X}^{\mathcal{A}} \otimes M \rightarrow M$ given by the dg- $\mathcal{E}_{X}^{\mathcal{A}}$-module structure coincides with the action $\mathcal{E}_{X}^{\mathbf{R}, f} \otimes \mu_{X}\left(\mathcal{O}_{X}^{t}\right) \rightarrow \mu_{X}\left(\mathcal{O}_{X}^{t}\right)$. Then, as recalled in Section 3 , extension and restriction of scalars yield an object $M^{\prime} \in \mathrm{D}^{b}\left(\mathcal{E}_{X}^{\mathbf{R}, f}\right)$ which represents $\mu_{X}\left(\mathcal{O}_{X}^{t}\right)$ with its $\mathcal{E}_{X}^{\mathbf{R}, f}$-action. So we conclude as in the non-tempered case.

Now we explain how we construct $\mathcal{E}_{X}^{\mathcal{A}}$ and $M$. The main step in the definition of $\mathcal{E}_{X}^{\mathbf{R}, f}$, as well as its action on $\mu h o m\left(F, \mathcal{O}_{X}\right)$, is the microlocal convolution product

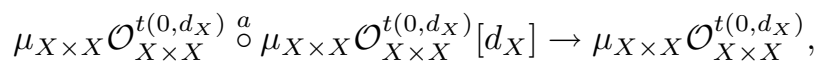

where $\stackrel{a}{\circ}$ denotes the composition of kernels. This is a morphism in the derived category. In order to obtain a true dg-algebra at the end, and not a complex with a product up to homotopy, we will represent the functor $\mu$ by a functor between categories of complexes which satisfies enough functorial properties so that the convolution also corresponds to a morphism of complexes.

Let us be more precise. The first step is the construction of injective resolutions with some functorial properties. For this we introduce a quasi-injective de Rham algebra, $\mathcal{A}$, below (quasi-injectivity is a property of ind-sheaves weaker than injectivity but sufficient to derive the usual functors). We use the construction of ind-sheaves from sheaves on the "subanalytic site" explained in [7. For a real analytic manifold $X$ the subanalytic site, $X_{s a}$, has for open subsets the subanalytic open subsets of $X$ and for coverings the locally finite coverings. On $X_{s a}$ it makes sense to consider the sheaf of tempered $\mathcal{C}^{\infty}$ functions, $\mathcal{C}_{X}^{\infty, t}$.

We consider the embedding $i_{X}: X=X \times\{0\} \rightarrow X \times \mathbf{R}$ and define a sheaf of $i$-forms on $X_{s a}, \mathcal{A}_{X}^{i}=i_{X}^{-1} \Gamma_{X \times \mathbf{R}_{>0}}\left(\mathcal{C}_{X \times \mathbf{R}}^{\infty, t(i)}\right)$. This gives a de Rham algebra $\mathcal{A}_{X}$ and it yields a quasi-injective resolution of $\mathbf{C}_{X_{s a}}$. We denote by $\operatorname{Mod}\left(\mathcal{A}_{X}\right)$ the category of sheaves of $\mathrm{dg}-\mathcal{A}_{X}$-modules. We have an obvious forgetful functor $\operatorname{For}_{X}^{\prime}: \operatorname{Mod}\left(\mathcal{A}_{X}\right) \rightarrow \mathrm{D}\left(\mathbf{C}_{X_{s a}}\right)$. We will prove that the operations needed in the construction of $(3)$ are defined in $\operatorname{Mod}\left(\mathcal{A}_{X}\right)$ and commute with For $_{X}^{\prime}$. Namely, for a morphism of manifolds $f: X \rightarrow Y$ we have functors $f^{*}$ and $f_{*}, f_{! !}$, of inverse and direct images of $\operatorname{dg}-\mathcal{A}$-modules. In some cases this gives a way to rep- 
resent the derived functors $f^{-1}$ and $\mathrm{R} f_{*}, \mathrm{R} f_{\text {!! }}$. For example, for $F \in \operatorname{Mod}\left(\mathcal{A}_{X}\right)$, $\operatorname{For}_{Y}^{\prime}\left(f_{! !}(F)\right) \simeq \mathrm{R} f_{! !}\left(\operatorname{For}_{X}^{\prime}(F)\right)$; if $f$ is smooth we also prove, for $G \in \operatorname{Mod}\left(\mathcal{A}_{Y}\right)$, $\operatorname{For}_{X}^{\prime}\left(f^{*} G\right) \simeq f^{-1}\left(\operatorname{For}_{Y}^{\prime}(G)\right)$. When $X$ is a complex manifold we also have a "Dolbeault resolution", $\mathbb{O}_{X}$, of $\mathcal{O}_{X}^{t}$ by a dg- $\mathcal{A}_{X}$-module which is locally free over $\mathcal{A}_{X}^{0}$.

Once we have these operations we define a microlocalization functor for $\mathrm{dg}-\mathcal{A}$ modules. Let us recall that the functor $\mu_{X}$ is given by composition with a kernel $L_{X} \in \mathrm{D}^{b}\left(\mathbf{C}_{\left(X \times T^{*} X\right)_{s a}}\right)$ : for $F \in \mathrm{D}^{b}\left(\mathbf{C}_{X_{s a}}\right)$ we have

$$
\mu_{X}(F)=L_{X} \circ F=\operatorname{R} p_{2 ! !}\left(L_{X} \otimes p_{1}^{-1} F\right) .
$$

We define a corresponding $\mathrm{dg}$ - $\mathcal{A}$-module, $L_{X}^{\mathcal{A}}$, outside the zero section of $T^{*} X$, i.e. over $X \times \dot{T}^{*} X$, which is quasi-isomorphic to $L_{X}$, and for a $\operatorname{dg}-\mathcal{A}_{X}$-module $F$ we set

$$
\mu_{X}^{\mathcal{A}}(F)=L_{X}^{\mathcal{A}} \circ F=p_{2 ! !}\left(L_{X}^{\mathcal{A}} \otimes_{\mathcal{A}} p_{1}^{*} F\right) .
$$

This functor is defined on the categories of complexes, i.e. it is a functor from $\operatorname{Mod}\left(\mathcal{A}_{X}\right)$ to $\operatorname{Mod}\left(\mathcal{A}_{\dot{T}^{*} X}\right)$. If $F$ has a finite 0 -presentation we show that $\mu_{X}^{\mathcal{A}}(F)$ is quasi-injective and represents $\mu_{X}(F)$ over $\dot{T}^{*} X$ : we have

$$
\operatorname{For}_{T^{*} X}^{\prime}\left(\mu_{X}^{\mathcal{A}}(F)\right) \simeq \mu_{X}\left(\operatorname{For}_{X}^{\prime}(F)\right)
$$

In particular, when $X$ is a complex manifold we obtain the $\mathrm{dg}-\mathcal{A}_{T^{*} X^{-}}$-module $\mu_{X}^{\mathcal{A}}\left(\mathbb{O}_{X}\right)$ which represents $\mu_{X}\left(\mathcal{O}_{X}^{t}\right)$ and can be used to compute $\mathrm{RHom}\left(\cdot, \mu_{X}\left(\mathcal{O}_{X}^{t}\right)\right)$.

With these tools in hand we define the sheaf $\mathcal{E}_{X}^{\mathcal{A}}$ mentioned above from $\mu^{\mathcal{A}}$, the same way $\mathcal{E}_{X}^{\mathbf{R}, f}$ was defined from $\mu$. The definition of the product involves a convolution product for $\mu^{\mathcal{A}}$. The kernel $L_{X}^{\mathcal{A}}$ has indeed the same functorial behavior as $L_{X}$ not with respect to all operations but at least those needed in the composition of kernels. We end up with a $\mathrm{dg}-\mathcal{A}_{T^{*} X}$-module $\mathcal{E}_{X}^{\mathcal{A}}$ which is a ring object in the category of $\mathrm{dg}-\mathcal{A}_{T^{*} X}$-modules and which represents $\mathcal{E}_{X}^{\mathbf{R}, f}$. In the same way we obtain a structure of $\mathcal{E}_{X}^{\mathcal{A}}$-module on $\mu_{X}^{\mathcal{A}}\left(\mathbb{O}_{X}\right)$, as desired. As said above this $\mathcal{E}_{X}^{\mathcal{A}}$-module gives a $\beta_{T^{*} X}\left(\mathcal{E}_{X}^{\mathbf{R}, f}\right)$-module by extension and restriction of scalars (here $\beta$ is the functor from sheaves to ind-sheaves which is left adjoint to $\alpha$ ). Our result is more precisely stated in Theorem 11.4

Theorem 1.1. There exists an ind-sheaf $\mathcal{O}_{X}^{\mu} \in \mathrm{D}\left(\beta_{\dot{T}^{*} X}\left(\mathcal{E}_{X}^{\mathbf{R}, f}\right)\right)$ which satisfies the following properties:

(i) the image of $\mathcal{O}_{X}^{\mu}$ in $\mathrm{D}\left(\mathbf{I}\left(\mathbf{C}_{\dot{T}^{*} X}\right)\right)$ under the forgetful functor is $\mu_{X} \mathcal{O}_{X}^{t}$,

(ii) for any $F \in \mathrm{D}^{-}\left(\mathbf{I}\left(\mathbf{C}_{X}\right)\right)$ the complex $\alpha_{T^{*} X} \mathrm{RIH} \mathcal{H o m}\left(\pi^{-1} F, \mathcal{O}_{X}^{\mu}\right)$ is naturally defined in $\mathrm{D}\left(\mathcal{E}_{X}^{\mathbf{R}, f}\right)$, over $\dot{T}^{*} X$. Its image in $\mathrm{D}\left(\mathbf{C}_{\dot{T}^{*} X}\right)$ under the forgetful functor is $T$ - $\mu$ hom $\left(F, \mathcal{O}_{X}\right)$ endowed with its action of $\mathcal{E}_{X}^{\mathbf{R}, f}$. 


\section{§2. Notations}

If $X$ is a manifold or a site and $R$ a sheaf of rings on $X$ we denote by $\operatorname{Mod}(R)$ the category of sheaves of $R$-modules on $X$. The corresponding category of complexes is $\mathrm{C}(R)$ and the derived category $\mathrm{D}(R)$; we use superscripts $b,+,-$ for the categories of complexes which are bounded, bounded from below, bounded from above. More generally, if $R$ is a sheaf of dg-algebras on $X$, then $\operatorname{Mod}(R)$ is the category of sheaves of dg-R-modules on $X$, and $\mathrm{D}(R)$ its derived category (see Section 3 ). In particular, if $X$ is a real analytic manifold this applies to the subanalytic site $X_{s a}$ whose definition is recalled in Section 4. We denote by $\rho_{X}$ or $\rho$ the natural morphism of sites $X \rightarrow X_{s a}$. We denote by $\mathbf{C}_{X}$ and $\mathbf{C}_{X_{s a}}$ the constant sheaves with coefficients $\mathbf{C}$ on $X$ and $X_{s a}$.

If $X$ is a manifold we denote by $\mathbf{I}\left(\mathbf{C}_{X}\right)$ the category of ind-sheaves of $\mathbf{C}_{X^{-}}$ vector spaces on $X$ (see Section 4 ), and by $\mathrm{D}\left(\mathbf{I}\left(\mathbf{C}_{X}\right)\right.$ ) its derived category. This category comes with a natural functor $\alpha_{X}$ or $\alpha: \mathbf{I}\left(\mathbf{C}_{X}\right) \rightarrow \operatorname{Mod}\left(\mathbf{C}_{X}\right)$ which corresponds to taking the limit. Its left adjoint is denoted $\beta_{X}$ or $\beta$.

The dimension of a (real) manifold $X$ is denoted $d_{X}$; if $X$ is a complex manifold its complex dimension is $d_{X}^{c}$.

For a morphism of manifolds $f: X \rightarrow Y$, we let $\omega_{X \mid Y}=f^{!} \mathbf{C}_{Y}$ be the relative dualizing complex. Hence $\omega_{X \mid Y}$ is an object of $\mathrm{D}^{b}\left(\mathbf{C}_{X}\right)$. If $Y$ is a point we simply write $\omega_{X}$; then $\omega_{X} \simeq \operatorname{or}_{X}\left[d_{X}\right]$, where $o r_{X}$ is the orientation sheaf of $X$. In fact, for $X$ connected $\omega_{X \mid Y}$ is always concentrated in one degree (since $X$ and $Y$ are manifolds), say $i$, and we will also use the notation $\omega_{X \mid Y}$ for the object of $\mathrm{C}^{b}\left(\mathbf{C}_{X}\right)$ which is $H^{i} \omega_{X \mid Y}$ in degree $i$ and 0 in other degrees. For an embedding of manifolds $i_{Z}: Z \hookrightarrow X$ we will often abuse notation and write $\omega_{Z \mid X}$ for $i_{Z *} \omega_{Z \mid X}$.

For a manifold $X$ we let $T X$ and $T^{*} X$ be the tangent and cotangent bundles and we denote by $\pi_{X}: T^{*} X \rightarrow X$ the projection. For a submanifold $Z \subset X$ we denote by $T_{Z} X$ and $T_{Z}^{*} X$ the normal and conormal bundles to $Z$. In particular $T_{X}^{*} X \simeq X$ is the zero section of $T^{*} X$ and we set $\dot{T}^{*} X=T^{*} X \backslash T_{X}^{*} X$. We denote by $\tilde{X}_{Z}$ the normal deformation of $Z$ in $X$ (see for example [5]). We recall that it contains $T_{Z} X$ and comes with a map $\tau: \tilde{X}_{Z} \rightarrow \mathbf{R}$ such that $\tau^{-1}(0)=T_{Z} X$ and $\tau^{-1}(r) \simeq X$ for $r \neq 0$. We also have another map $p: \tilde{X}_{Z} \rightarrow X$ such that $p^{-1}(z)=\left(T_{Z} X\right)_{z} \cup\{z\} \times \mathbf{R}$ for $z \in Z$ and $p^{-1}(x) \simeq \mathbf{R} \backslash\{0\}$ for $x \in X \backslash Z$. We set $\Omega=\tau^{-1}\left(\mathbf{R}_{>0}\right)$.

For a morphism of manifolds $f: X \rightarrow Y$, the derivative of $f$ gives the morphisms

$$
T^{*} X \stackrel{f_{d}}{\longleftarrow} X \times_{Y} T^{*} Y \stackrel{f_{\pi}}{\longrightarrow} T^{*} Y .
$$

For two manifolds $X, Y$, and $F \in \mathrm{D}^{+}\left(\mathbf{C}_{X}\right), G \in \mathrm{D}^{+}\left(\mathbf{C}_{Y}\right)$, we set $F \otimes G=$ $p_{1}^{-1} F \otimes p_{2}^{-1} G$, where $p_{i}$ is the projection from $X \times Y$ to the $i^{\text {th }}$ factor. For three 
manifolds $X, Y, Z$ and "kernels" $K \in \mathrm{D}^{+}\left(\mathbf{C}_{X \times Y}\right), L \in \mathrm{D}^{+}\left(\mathbf{C}_{Y \times Z}\right)$ we denote the "composition of kernels" by $K \circ L=\mathrm{R} p_{23 !}\left(p_{12}^{-1} K \otimes p_{23}^{-1} L\right)$, where $p_{i j}$ is the projection from $X \times Y \times Z$ to the $i^{\text {th }} \times j^{\text {th }}$ factors.

\section{§3. dg-algebras}

In this section we recall some facts about (sheaves of) dg-algebras and their derived categories. We refer the reader to [2].

A $d g$-algebra $A$ is a $\mathbf{Z}$-graded algebra with a differential $d_{A}$ of degree +1 . A $d g$-A-module $M$ is a graded $A$-module with a differential $d_{M}$ such that, for homogeneous elements $a \in A^{i}, m \in M^{j}, d_{M}(a \cdot m)=d_{A}(a) \cdot m+(-1)^{i} a \cdot d_{M} m$.

We consider a site $X$ and a sheaf of dg-algebras $A_{X}$ on $X$. We denote by $\operatorname{Mod}\left(A_{X}\right)$ the category of (left) $\operatorname{dg}-A_{X}$-modules. We let $\widetilde{A}_{X}$ be the graded algebra underlying $A_{X}$ (i.e. forgetting the differential). A morphism $f: M \rightarrow N$ in $\operatorname{Mod}\left(A_{X}\right)$ is said to be null homotopic if there exists an $\widetilde{A}_{X}$-linear morphism $s: M \rightarrow N[-1]$ such that $f=s d_{M}+d_{N} s$. The homotopy category, $\mathrm{K}\left(A_{X}\right)$, has for objects those of $\operatorname{Mod}\left(A_{X}\right)$ and for sets of morphisms those of $\operatorname{Mod}\left(A_{X}\right)$ quotiented by null homotopic morphisms. A morphism in $\operatorname{Mod}\left(A_{X}\right)\left(\operatorname{or} \mathrm{K}\left(A_{X}\right)\right)$ is a quasi-isomorphism if it induces isomorphisms on the cohomology groups. Finally, the derived category $\mathrm{D}\left(A_{X}\right)$ is the localization of $\mathrm{K}\left(A_{X}\right)$ by quasi-isomorphisms.

Derived functors can be defined in this setting, in particular the tensor product $\cdot \otimes_{A_{X}}^{L} \cdot$ If $\phi: A_{X} \rightarrow B_{X}$ is a morphism of sheaves of dg-algebras we obtain the extension of scalars $\phi^{*}: \mathrm{D}\left(A_{X}\right) \rightarrow \mathrm{D}\left(B_{X}\right), M \mapsto B_{X} \otimes_{A_{X}}^{L} M$, which is left adjoint to the natural restriction of scalars $\phi_{*}: \mathrm{D}\left(B_{X}\right) \rightarrow \mathrm{D}\left(A_{X}\right)$. By [2, Theorem 10.12.5.1], if $\phi$ induces an isomorphism $H(A) \stackrel{\sim}{\rightarrow} H(B)$ then these functors of restriction and extension of scalars are mutually inverse equivalences of categories $\mathrm{D}\left(A_{X}\right) \simeq \mathrm{D}\left(B_{X}\right)$.

Some dg-algebras considered in this paper will appear as ring objects in categories of complexes. We recall briefly what this means. We let $\mathcal{C}$ be a tensor category with unit $\underline{\mathbf{C}}\left(\mathcal{C}\right.$ will be $\mathrm{D}\left(\mathbf{C}_{Y}\right), \mathrm{D}\left(\mathbf{I}\left(\mathbf{C}_{Y}\right)\right)$ or $\operatorname{Mod}\left(\mathcal{A}_{Y}\right)$ for some manifold $Y$ and the unit is $\left.\underline{\mathbf{C}}=\mathbf{C}_{Y}\right)$.

Definition 3.1. A ring in $\mathcal{C}$ is a triplet $(A, m, \varepsilon)$ where $A \in \mathcal{C}, m: A \otimes A \rightarrow A$ and $\varepsilon: \underline{\mathbf{C}} \rightarrow A$ are morphisms in $\mathcal{C}$ such that the following diagrams commute:
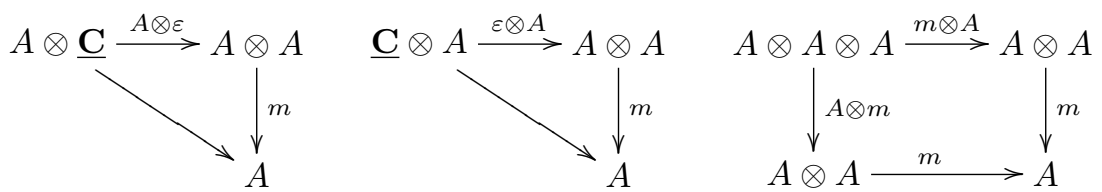
In the same way, for such a "ring" $(A, m, \varepsilon)$, an action of $A$ on $M \in \mathcal{C}$ is a morphism $\alpha: A \otimes M \rightarrow M$ compatible with $m$ and $\varepsilon$. The pairs $(M, \alpha)$ of this type form a category, where morphisms from $(M, \alpha)$ to $\left(M^{\prime}, \alpha^{\prime}\right)$ are morphisms from $M$ to $M^{\prime}$ commuting with the action.

If $E_{X}$ is a sheaf of (usual) algebras on $X$ we may consider $E_{X}$ as a ring object in $\mathrm{D}\left(\mathbf{C}_{X}\right)$ and we denote by $\mathrm{D}_{E_{X}}\left(\mathbf{C}_{X}\right)$ the category of "objects of $\mathrm{D}\left(\mathbf{C}_{X}\right)$ with $E_{X}$-action" as above.

We consider again a sheaf $A_{X}$ of dg-algebras on $X$. We assume that its cohomology sheaves are 0 except in degree 0 and we set $E_{X}=H^{0}\left(A_{X}\right)$. Hence, if we forget the structures and view $A_{X}, E_{X}$ as objects of $\mathrm{D}\left(\mathbf{C}_{X}\right)$ we have isomorphisms $A_{X} \longleftarrow \tau_{\leq 0} A_{X} \stackrel{\sim}{\rightarrow} E_{X}$ (where $\tau_{\leq 0}, \tau_{\geq 0}$ denote the truncation functors). We note that $\tau_{\leq 0} A_{X}=\cdots \rightarrow A_{X}^{-1} \rightarrow$ ker $d_{0} \rightarrow 0$ is a sub-dg-algebra of $A_{X}$ (whereas $\tau_{\geq 0} A_{X}$ has no obvious structure of dg-algebra). The multiplications of $A_{X}$ and $E_{X}$ induce morphisms in $\mathrm{D}\left(\mathbf{C}_{X}\right): A_{X} \otimes A_{X} \rightarrow A_{X}, E_{X} \otimes E_{X} \rightarrow E_{X}$. These morphisms coincide under the identification $A_{X} \simeq E_{X}$. Hence $A_{X}$ and $E_{X}$ are isomorphic as ring objects in $\mathrm{D}\left(\mathbf{C}_{X}\right)$.

For $M \in \mathrm{D}\left(A_{X}\right)$ the structure of $A_{X}$-module induces a morphism in $\mathrm{D}\left(\mathbf{C}_{X}\right)$ : $\alpha: E_{X} \otimes M \simeq A_{X} \otimes M \rightarrow M$. Then $\alpha$ is an action of $E_{X}$ on $M$. In this way we obtain a forgetful functor $F_{A_{X}}: \mathrm{D}\left(A_{X}\right) \rightarrow \mathrm{D}_{E_{X}}\left(\mathbf{C}_{X}\right)$.

Lemma 3.2. Let $A_{X}$ be a sheaf of dg-algebras with cohomology sheaves concentrated in degree 0 and set $E_{X}=H^{0}\left(A_{X}\right)$. Let $\phi: A_{X} \rightarrow B_{X}$ be a morphism of sheaves of dg-algebras such that $\phi$ induces an isomorphism $H(A) \stackrel{\sim}{\rightarrow} H(B)$. Then we have isomorphisms of functors $F_{A_{X}} \circ \phi_{*} \simeq F_{B_{X}}$ and $F_{B_{X}} \circ \phi^{*} \simeq F_{A_{X}}$.

Proof. The first isomorphism is obvious and the second one follows because $\phi_{*}$ and $\phi^{*}$ are inverse equivalences of categories.

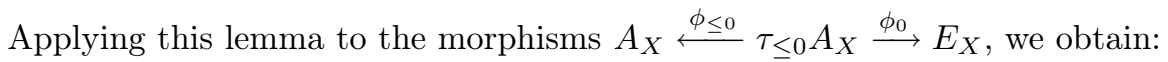

Corollary 3.3. With the hypothesis of the above lemma, we have the commutative diagram

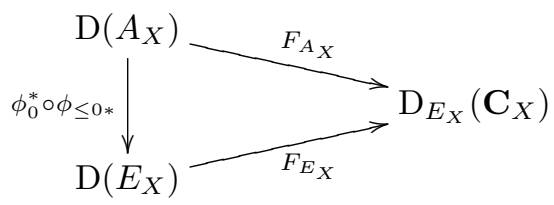

In particular, for $M \in \mathrm{D}_{E_{X}}\left(\mathbf{C}_{X}\right)$, if there exists $N \in \mathrm{D}\left(A_{X}\right)$ such that $F_{A_{X}}(N) \simeq M$ then there exists $N^{\prime} \in \mathrm{D}\left(E_{X}\right)$ such that $F_{E_{X}}\left(N^{\prime}\right) \simeq M$. 


\section{§4. Ind-sheaves and subanalytic site}

We recall briefly some definitions and results of [7] about ind-sheaves. To define the ind-sheaves we are interested in we will use the "subanalytic site" as in [7, where it is introduced to deal with tempered $\mathcal{C}^{\infty}$ functions. It is studied in more detail in [10].

\section{§4.1. Ind-sheaves}

For a category $\mathcal{C}$ we denote by $\mathcal{C}^{\wedge}$ the category of functors from $\mathcal{C}^{o p}$ to the category of sets. It comes equipped with the "Yoneda embedding", $h: \mathcal{C} \rightarrow \mathcal{C}^{\wedge}$, $X \mapsto \operatorname{Hom}_{\mathcal{C}}(\cdot, X)$. The category $\mathcal{C}^{\wedge}$ admits small inductive limits but, in general, even if $\mathcal{C}$ also admits such limits, the functor $h$ may not commute with inductive limits. We denote by "lim" the inductive limit taken in the category $\mathcal{C}^{\wedge}$.

An ind-object in $\mathcal{C}$ is an object of $\mathcal{C}^{\wedge}$ which is isomorphic to "lim" $i$ for some functor $i: I \rightarrow \mathcal{C}$, with $I$ a small filtrant category. We denote by $\overrightarrow{\operatorname{Ind}}(\mathcal{C})$ the full subcategory of $\mathcal{C}^{\wedge}$ of ind-objects.

Let $X$ be a real analytic manifold, $\operatorname{Mod}\left(\mathbf{C}_{X}\right)$ the category of sheaves of $\mathbf{C}$ vector spaces on $X, \operatorname{Mod}_{\mathbf{R}-c}\left(\mathbf{C}_{X}\right)$ the subcategory of $\mathbf{R}$-constructible sheaves, and $\operatorname{Mod}^{c}\left(\mathbf{C}_{X}\right)$ and $\operatorname{Mod}_{\mathbf{R}-c}^{c}\left(\mathbf{C}_{X}\right)$ their respective full subcategories of objects with compact support. We define, as in [7],

$$
\mathbf{I}\left(\mathbf{C}_{X}\right)=\operatorname{Ind}\left(\operatorname{Mod}^{c}\left(\mathbf{C}_{X}\right)\right) \quad \text { and } \quad \mathbf{I}_{\mathbf{R}-c}\left(\mathbf{C}_{X}\right)=\operatorname{Ind}\left(\operatorname{Mod}_{\mathbf{R}-c}^{c}\left(\mathbf{C}_{X}\right)\right) \text {. }
$$

There are exact embeddings $I_{\tau}: \mathbf{I}_{\mathbf{R}-c}\left(\mathbf{C}_{X}\right) \rightarrow \mathbf{I}\left(\mathbf{C}_{X}\right)$ and $\iota_{X}: \operatorname{Mod}\left(\mathbf{C}_{X}\right) \rightarrow \mathbf{I}\left(\mathbf{C}_{X}\right)$,

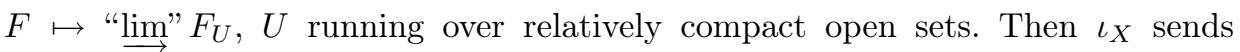
$\operatorname{Mod}_{\mathbf{R}-c}\left(\mathbf{C}_{X}\right)$ into $\mathbf{I}_{\mathbf{R}-c}\left(\mathbf{C}_{X}\right)$.

The functor $\iota_{X}$ has an exact left adjoint functor $\alpha_{X}: \mathbf{I}\left(\mathbf{C}_{X}\right) \rightarrow \operatorname{Mod}\left(\mathbf{C}_{X}\right)$, "lim" ${ }_{i \in I} F_{i} \mapsto \underline{\lim }_{i \in I} F_{i}$. Since $\iota_{X}$ is fully faithful, we have $\alpha_{X} \circ \iota_{X} \simeq$ id.

The functor $\alpha_{X}$ admits an exact fully faithful left adjoint $\beta_{X}: \operatorname{Mod}\left(\mathbf{C}_{X}\right) \rightarrow$ $\mathbf{I}\left(\mathbf{C}_{X}\right)$. We have $\alpha_{X} \circ \beta_{X} \simeq$ id. For $Z \subset X$ a closed subset we have

$$
\beta_{X}\left(\mathbf{C}_{Z}\right) \simeq \underset{W}{ } \underset{\text { lim" }}{ } \mathbf{C}_{\bar{W}}, \quad W \text { open, } Z \subset W \subset X
$$

We write $\alpha, \beta$ for $\alpha_{X}, \beta_{X}$ when the context is clear. The machinery of Grothendieck's six operations also applies in this context. There are not enough injectives in $\mathbf{I}\left(\mathbf{C}_{X}\right)$, but enough "quasi-injectives" (see [7] and [8]): $F \in \mathbf{I}\left(\mathbf{C}_{X}\right)$ is quasi-injective if the functor $\operatorname{Hom}(\cdot, F)$ is exact on $\operatorname{Mod}^{c}\left(\mathbf{C}_{X}\right)$. The quasi-injective objects are sufficient to derive the usual functors. In particular, for a morphism of manifolds 
$f: X \rightarrow Y$ we have the functors

$$
\begin{aligned}
& f^{-1}, f^{!}: \mathrm{D}^{b}\left(\mathbf{I}\left(\mathbf{C}_{Y}\right)\right) \rightarrow \mathrm{D}^{b}\left(\mathbf{I}\left(\mathbf{C}_{X}\right)\right), \\
& \mathrm{R} f_{*}, \mathrm{R} f_{! !}: \mathrm{D}^{b}\left(\mathbf{I}\left(\mathbf{C}_{X}\right)\right) \rightarrow \mathrm{D}^{b}\left(\mathbf{I}\left(\mathbf{C}_{Y}\right)\right), \\
& \mathrm{R} \mathcal{H} \text { Hom: } \mathrm{D}^{b}\left(\mathbf{I}\left(\mathbf{C}_{X}\right)\right)^{o p} \times \mathrm{D}^{b}\left(\mathbf{I}\left(\mathbf{C}_{X}\right)\right) \rightarrow \mathrm{D}^{+}\left(\mathbf{I}\left(\mathbf{C}_{X}\right)\right) \text {, } \\
& \otimes: \mathrm{D}^{b}\left(\mathbf{I}\left(\mathbf{C}_{X}\right)\right) \times \mathrm{D}^{b}\left(\mathbf{I}\left(\mathbf{C}_{X}\right)\right) \rightarrow \mathrm{D}^{b}\left(\mathbf{I}\left(\mathbf{C}_{X}\right)\right),
\end{aligned}
$$

and also RHom $=\alpha \mathrm{R} \mathcal{H} \mathcal{H}$ om: $\mathrm{D}^{b}\left(\mathbf{I}\left(\mathbf{C}_{X}\right)\right)^{o p} \times \mathrm{D}^{b}\left(\mathbf{I}\left(\mathbf{C}_{X}\right)\right) \rightarrow \mathrm{D}^{+}\left(\mathbf{C}_{X}\right)$.

It will be convenient for us to use the equivalence of categories given in [7] between $\mathbf{I}_{\mathbf{R}-c}\left(\mathbf{C}_{X}\right)$ and sheaves on the subanalytic site, defined below.

\section{§4.2. Subanalytic site}

In this subsection $X$ is a real analytic manifold. The open sets of the site $X_{s a}$ are the subanalytic open subsets of $X$. A family $\bigcup_{i \in I} U_{i}$ of such open sets is a covering of $U$ if and only if, for any compact subset $K$, there exists a finite subfamily $J$ of $I$ with $K \cap \bigcup_{i \in J} U_{i}=K \cap U$. We denote by $\operatorname{Mod}\left(\mathbf{C}_{X_{s a}}\right)$ the category of sheaves of $\mathbf{C}$-vector spaces on $X_{s a}$.

We have a morphism of sites $\rho_{X}: X \rightarrow X_{s a}$ (where $X$ also denotes the site naturally associated to the topological space $X$ ). We just write $\rho$ if there is no risk of confusion. In particular we have adjoint functors $\rho_{*}: \operatorname{Mod}\left(\mathbf{C}_{X}\right) \rightarrow \operatorname{Mod}\left(\mathbf{C}_{X_{s a}}\right)$ and $\rho^{-1}: \operatorname{Mod}\left(\mathbf{C}_{X_{s a}}\right) \rightarrow \operatorname{Mod}\left(\mathbf{C}_{X}\right)$. The functor $\rho^{-1}$ is exact, $\rho_{*}$ is left exact and fully faithful and $\rho^{-1} \circ \rho_{*}=$ id. We denote by $\rho_{c *}$ the restriction of $\rho_{*}$ to $\operatorname{Mod}_{\mathbf{R}-c}\left(\mathbf{C}_{X}\right)$. Then $\rho_{c *}$ is exact and for $F \in \operatorname{Mod}_{\mathbf{R}-c}\left(\mathbf{C}_{X}\right)$ we usually write $F$ instead of $\rho_{c *} F$. The functor $\rho_{c *}$ induces an equivalence of categories (see [7, Theorem 6.3.5])

$$
\lambda: \mathbf{I}_{\mathbf{R}-c}\left(\mathbf{C}_{X}\right) \rightarrow \operatorname{Mod}\left(\mathbf{C}_{X_{s a}}\right), \quad \underset{i}{\stackrel{\lim }{\longrightarrow}} F_{i} \mapsto \underset{i}{\lim } \rho_{c *}\left(F_{i}\right) .
$$

Through this equivalence the functor $\rho^{-1}$ corresponds to $\alpha$ and it also admits an exact left adjoint functor corresponding to $\beta$. When dealing with the analytic site we will use the notation $\rho_{!}: \operatorname{Mod}\left(\mathbf{C}_{X}\right) \rightarrow \operatorname{Mod}\left(\mathbf{C}_{X_{s a}}\right)$ for this functor. For example, (44) gives $\rho ! \mathbf{C}_{Z} \simeq \lim _{Z \subset W} \mathbf{C}_{\bar{W}}$ ( $W$ open subanalytic). We note the commutative diagrams

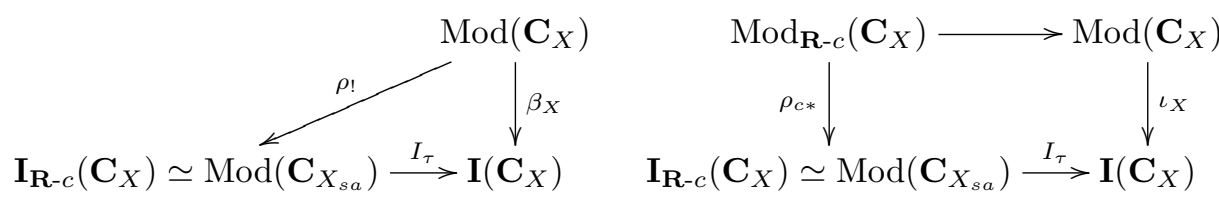

The functors appearing in these diagrams are exact and induce similar commutative diagrams at the level of derived categories. 
The functor $\mathcal{H}$ om is defined on $\operatorname{Mod}\left(\mathbf{C}_{X_{s a}}\right)$ just as on every site and we set, for $Z \subset X$ a locally closed subanalytic subset,

$$
\Gamma_{Z}(F)=\mathcal{H o m}\left(\rho_{*} \mathbf{C}_{Z}, F\right), \quad F_{Z}=F \otimes \rho_{*} \mathbf{C}_{Z} .
$$

The functors $\rho_{*}$ and $\mathcal{H}$ om commute, hence $\rho_{*}$ and $\Gamma_{Z}$ also commute. For subanalytic open subsets $U, V \subset X$ we have $\Gamma_{U}(F)(V)=F(U \cap V)$.

As for ind-sheaves, a notion weaker than injective is defined in [10]: $F \in$ $\operatorname{Mod}\left(\mathbf{C}_{X_{s a}}\right)$ is quasi-injective if $\operatorname{Hom}(\cdot, F)$ is exact on $\rho_{*} \operatorname{Mod}_{\mathbf{R}-c}^{c}\left(\mathbf{C}_{X}\right)$. In fact, since we consider coefficients in a field it is equivalent to require that for any subanalytic open subsets $U \subset V$ with compact closure, $\Gamma(V ; F) \rightarrow \Gamma(U ; F)$ is surjective. Quasiinjective sheaves are sufficient to derive usual left exact functors. In particular we obtain $\mathrm{R} \mathcal{H}$ om, $\mathrm{R} \Gamma_{Z}$, and they commute with $\mathrm{R} \rho_{*}$. We note the following identity (which has no equivalent on the classical site): for $F \in \mathrm{D}_{\mathbf{R}-\mathrm{c}}^{\mathrm{b}}\left(\mathbf{C}_{X}\right), H \in \mathrm{D}^{+}\left(\mathbf{C}_{X}\right)$, $G \in \mathrm{D}^{+}\left(\mathbf{C}_{X_{s a}}\right)$,

$$
\mathrm{R} \mathcal{H o m}\left(\mathrm{R} \rho_{*} F, G\right) \otimes \rho_{!} H \simeq \mathrm{RHom}\left(\mathrm{R} \rho_{*} F, G \otimes \rho_{!} H\right) \quad \text { in } \mathrm{D}^{+}\left(\mathbf{C}_{X_{s a}}\right) .
$$

We also have another related result (see [10, Proposition 1.1.3]): for $\left\{F_{i}\right\}_{i \in I}$ a filtrant inductive system in $\operatorname{Mod}\left(\mathbf{C}_{X_{s a}}\right)$ and $U \subset X$ a subanalytic open subset,

$$
\underset{i}{\lim _{i}} \mathrm{R} \Gamma_{U}\left(F_{i}\right) \stackrel{\sim}{\longrightarrow} \Gamma_{U}\left(\underset{i}{\lim } F_{i}\right) .
$$

For a morphism $f: X \rightarrow Y$ there are the usual direct and inverse image functors $f_{*}, f^{-1}$ on the subanalytic sites and also, as in the case of ind-sheaves, a notion of proper direct image $f_{! !}$, with a behavior slightly different from the behavior of $f$ ! on the classical site. The functor $f^{-1}$ is exact and $f_{*}, f_{! !}$admit derived functors. We quote in particular: for $F \in \mathrm{D}^{+}\left(\mathbf{C}_{X_{s a}}\right), G \in \mathrm{D}_{\mathbf{R}-c}^{b}\left(\mathbf{C}_{Y}\right)$ (we write $G$ for $\rho_{*} G$ ),

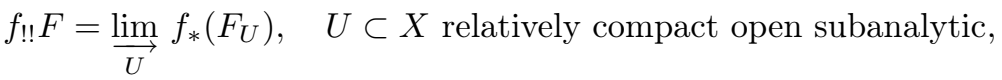

$$
\begin{aligned}
& f_{! !} F=\underset{K}{\lim _{*}} f_{*}\left(\Gamma_{K} F\right), \quad K \subset X \text { compact subanalytic, } \\
& \mathrm{R} f_{! !} \mathrm{R} \mathcal{H o m}\left(f^{-1} G, F\right) \stackrel{\sim}{\rightarrow} \operatorname{RHom}\left(G, \mathrm{R} f_{! !} F\right), \\
& \mathrm{R} f_{! !} \mathrm{R} \Gamma_{f^{-1} U} F \stackrel{\mathrm{R}}{\rightarrow} \Gamma_{U} \mathrm{R} f_{! !} F .
\end{aligned}
$$

The derived functor $\mathrm{R} f ! !: \mathrm{D}^{+}\left(\mathbf{C}_{X_{s a}}\right) \rightarrow \mathrm{D}^{+}\left(\mathbf{C}_{Y_{s a}}\right)$ admits a right adjoint $f^{!}$. The notation is the same as in the classical case because of the commutation relation $f^{!} \circ \mathrm{R} \rho_{*} \simeq \mathrm{R} \rho_{*} \circ f^{!}$. Hence $f^{!} \mathbf{C}_{Y_{s a}} \simeq \rho_{*} \omega_{X \mid Y}$ and we will usually write $\omega_{X \mid Y}$ for $\rho_{*} \omega_{X \mid Y}$. The adjunction morphism between $f_{\text {!! }}$ and $f^{!}$induces the integration morphism

$$
\operatorname{int}_{f}: \mathrm{R} f_{! !}\left(\omega_{X \mid Y}\right) \rightarrow \mathbf{C}_{Y_{s a}} .
$$




\section{§4.3. "Soft" sheaves}

In this subsection $X$ is a real analytic manifold and $X_{s a}$ is the corresponding subanalytic site. Though we are not in a framework of sheaves on a locally compact space, we may introduce a notion of soft sheaves on the subanalytic site which are acyclic for direct image functors.

Definition 4.1. A sheaf $F \in \operatorname{Mod}\left(\mathbf{C}_{X_{s a}}\right)$ is soft if for any closed subanalytic subset $Z \subset X$ and any subanalytic open subset $U \subset X$ the natural morphism $\Gamma(U ; F) \rightarrow \Gamma\left(U ; F_{Z}\right)$ is surjective.

As in the case of sheaves on a reasonable topological space,

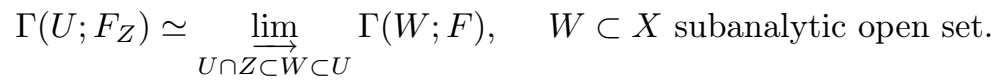

It follows that quasi-injective sheaves are soft. We also note that if $F$ is soft and $Z \subset X$ is a closed subanalytic subset then $F_{Z}$ is soft.

Lemma 4.2. Let $U=\bigcup_{i \in \mathbf{N}} U_{i}$ be a locally finite covering by subanalytic open subsets of $X$. There exist subanalytic open subsets of $X, V_{i} \subset U_{i}, i \in \mathbf{N}$, such that $U=\bigcup_{i \in \mathbf{N}} V_{i}$ and $U \cap \overline{V_{i}} \subset U_{i}$.

Proof. We choose an analytic distance $d$ on $X$ and we define $V_{n}$ inductively such that $U=\bigcup_{i \leq n} V_{i} \cup \bigcup_{j>n} U_{j}$ and $U \cap \overline{V_{n}} \subset U_{n}$. We start with $V_{-1}=\emptyset$ and assume $V_{i}, i<n$, is built. We set $W_{n}=U_{n} \backslash\left(\bigcup_{i<n} V_{i} \cup \bigcup_{j>n} U_{j}\right)$, which is subanalytic because the covering is locally finite, and

$$
V_{n}=\left\{x \in U_{n} ; d\left(x, W_{n}\right)<d\left(x, \partial U_{n}\right)\right\} .
$$

We have $V_{n} \subset U_{n}$. Since $d$ is analytic the functions $d(\cdot, Z), Z \subset X$ subanalytic, are continuous functions with subanalytic graphs (see [3]). It follows that $V_{n}$ is a subanalytic open subset of $X$. By construction $W_{n} \subset V_{n}$ and we deduce by induction that $U=\bigcup_{i \leq n} V_{i} \cup \bigcup_{j>n} U_{j}$. Since the covering is locally finite this gives $U=\bigcup_{i \in \mathbf{N}} V_{i}$.

It remains to prove that $U \cap \overline{V_{n}} \subset U_{n}$. If this is false there exists $x_{0} \in$ $U \cap \overline{V_{n}} \cap \partial U_{n}$. Since $x_{0} \in U$ but $x_{0} \notin U_{n}$ we have $x_{0} \in \bigcup_{i<n} V_{i} \cup \bigcup_{j>n} U_{j}$. Hence $\delta=d\left(x_{0}, W_{n}\right)>0$ and the ball $B\left(x_{0}, \delta / 2\right)$ does not meet $V_{n}$. In particular $x_{0} \notin \overline{V_{n}}$, which is a contradiction.

Proposition 4.3. Let $0 \rightarrow F^{\prime} \stackrel{u}{\rightarrow} F \stackrel{v}{\rightarrow} F^{\prime \prime} \rightarrow 0$ be an exact sequence in $\operatorname{Mod}\left(\mathbf{C}_{X_{s a}}\right)$ with $F^{\prime}$ soft. Then for any open subanalytic subset $U \subset X$ the morphisms

$$
\Gamma(U ; F) \rightarrow \Gamma\left(U ; F^{\prime \prime}\right) \quad \text { and } \underset{K}{\underset{K}{\lim }} \Gamma_{K}(U ; F) \rightarrow \underset{K}{\lim _{K}} \Gamma_{K}\left(U ; F^{\prime \prime}\right),
$$

where $K$ runs over the compact subanalytic subsets of $X$, are surjective. 
Proof. (i) We first consider a section $s \in \Gamma\left(U ; F^{\prime \prime}\right)$. We may find a locally finite covering $U=\bigcup_{i \in \mathbf{N}} U_{i}$ and $s_{i} \in \Gamma\left(U_{i} ; F\right)$ such that $u\left(s_{i}\right)=\left.s\right|_{U_{i}}$. By Lemma 4.2 there exists a subcovering $U=\bigcup_{i \in \mathbf{N}} V_{i}$ with $U \cap \overline{V_{i}} \subset U_{i}$.

We set $Z_{n}=\bigcup_{i=0}^{n} \overline{V_{i}}$ and prove by induction on $n$ that there exists a section $\tilde{s}_{n} \in \Gamma\left(U ; F_{Z_{n}}\right)$ such that $v\left(\tilde{s}_{n}\right)=\left.s\right|_{Z_{n}}$ and $\left.\tilde{s}_{n}\right|_{Z_{n-1}}=\tilde{s}_{n-1}$.

This is clear for $n=0$ and we assume it is proved for $n$. We set $t_{n}=$ $\left.\left(\tilde{s}_{n}-s_{n+1}\right)\right|_{Z_{n} \cap \overline{V_{n+1}}}$. Then $v\left(t_{n}\right)=0$ so that $t_{n}$ belongs to $\Gamma\left(U ; F_{Z_{n} \cap \overline{V_{n+1}}}^{\prime}\right)$ and by hypothesis we may extend it to $t \in \Gamma\left(U ; F^{\prime}\right)$. Now we define $\tilde{s}_{n+1} \in \Gamma\left(U ; F_{Z_{n+1}}\right)$ by $\left.\tilde{s}_{n+1}\right|_{Z_{n}}=\tilde{s}_{n}$ and $\left.\tilde{s}_{n+1}\right|_{\overline{V_{n+1}}}=s_{n+1}+u(t)$. The $\tilde{s}_{n}$ glue together into a section $\tilde{s} \in \Gamma(U ; F)$ such that $v(\tilde{s})=s$, which proves the surjectivity of the first morphism.

(ii) Now we consider a compact $K$ and $s \in \Gamma_{K}\left(U ; F^{\prime \prime}\right)$. We choose an open subanalytic subset $V$ such that $K \subset V$ and $K^{\prime}=\bar{V}$ is compact. We set $Z=X \backslash V$. We have just seen that we may find $\tilde{s} \in \Gamma(U ; F)$ such that $v(\tilde{s})=s$. Hence $v\left(\left.\tilde{s}\right|_{Z}\right)=0$ so that $\left.\tilde{s}\right|_{Z} \in \Gamma\left(U ; F_{Z}^{\prime}\right)$ and we may extend $\left.\tilde{s}\right|_{Z}$ to $t \in \Gamma\left(U ; F^{\prime}\right)$. Then $\hat{s}=\tilde{s}-u(t)$ satisfies supp $\hat{s} \subset K^{\prime}$ and $v(\hat{s})=s$.

Corollary 4.4. If $0 \rightarrow F^{\prime} \rightarrow F \rightarrow F^{\prime \prime} \rightarrow 0$ is an exact sequence in $\operatorname{Mod}\left(\mathbf{C}_{X_{s a}}\right)$ with $F^{\prime}$ and $F$ soft, then $F^{\prime \prime}$ is also soft.

Proof. For $Z \subset X$ a subanalytic closed subset we have the exact sequence $0 \rightarrow$ $F_{Z}^{\prime} \rightarrow F_{Z} \rightarrow F_{Z}^{\prime \prime} \rightarrow 0$ and $F_{Z}^{\prime}, F_{Z}$ still are soft. Hence Proposition 4.3 implies that, for any subanalytic open subset $U \subset X$, the morphisms $\Gamma(U ; F) \rightarrow \Gamma\left(U ; F^{\prime \prime}\right)$ and $\Gamma\left(U ; F_{Z}\right) \rightarrow \Gamma\left(U ; F_{Z}^{\prime \prime}\right)$ are surjective. Now it follows from the definition that $F^{\prime \prime}$ is soft.

Corollary 4.5. Let $f: X \rightarrow Y$ be a morphism of analytic manifolds, and $U \subset X$ an open subanalytic subset. Then soft sheaves in $\operatorname{Mod}\left(\mathbf{C}_{X_{\text {sa }}}\right)$ are acyclic for the functors $\Gamma(U ; \cdot), \lim _{K} \Gamma_{K}(U ; \cdot), K$ running over the compact subsets of $X, \Gamma_{U}, f_{*}$ and $f_{! !}$.

Proof. For the first two functors this follows from Proposition 4.3 and Corollary 4.4. This implies the result for the other functors.

\section{§4.4. Tempered functions}

Here we recall the definition of tempered $\mathcal{C}^{\infty}$ functions. We also state a tempered de Rham lemma on the subanalytic site, which is actually a reformulation of results of 4. In this subsection $X$ is a real analytic manifold.

Definition 4.6. A $\mathcal{C}^{\infty}$ function $f$ defined on an open set $U$ has polynomial growth at $p \in X$ if there exist a compact neighborhood $K$ of $p$ and $C, N>0$ such that for all $x \in K \cap U,|f(x)|<C d(x, K \backslash U)^{-N}$, for a distance $d$ defined through some coordinate system around $p$. 
We say that $f$ is tempered if all its derivatives have polynomial growth at any point. In [7] it is proved, using results of Łojasiewicz, that these functions define a subsheaf $\mathcal{C}_{X}^{\infty, t}$ of $\rho_{*} \mathcal{C}_{X}^{\infty}$ on $X_{s a}$.

We denote by $\Omega_{X}^{t, i}$ the sheaf on $X_{s a}$ of forms of degree $i$ with tempered coefficients. We obtain as usual a sheaf of dg-algebras on $X_{s a}$, the tempered de Rham algebra $\Omega_{X}^{t}=0 \rightarrow \Omega_{X}^{t, 0} \rightarrow \cdots \rightarrow \Omega_{X}^{t, n} \rightarrow 0$.

Lemma 4.7. The tempered de Rham algebra is a resolution of the constant sheaf on the subanalytic site, i.e. we have an exact sequence on $X_{s a}$,

$$
0 \rightarrow \mathbf{C}_{X_{s a}} \rightarrow \Omega_{X}^{t, 0} \rightarrow \cdots \rightarrow \Omega_{X}^{t, n} \rightarrow 0 .
$$

Proof. This is equivalent to saying that for any $F \in \mathrm{D}_{\mathbf{R}-c}^{b}\left(\mathbf{C}_{X}\right)$ we have

$$
\operatorname{RHom}\left(\rho_{*} F, \mathbf{C}_{X_{s a}}\right) \simeq \operatorname{RHom}\left(\rho_{*} F, \Omega_{X}^{t}\right) .
$$

Actually this is Proposition 4.6 of [4], except that it is not stated in this language, and that it is given for tempered distributions instead of tempered $\mathcal{C}^{\infty}$ functions. We let $\mathcal{C}_{X}^{\omega}$ be the sheaf of real analytic functions and $\mathcal{D}_{X}$ the sheaf of linear differential operators with coefficients in $\mathcal{C}_{X}^{\omega}$. Using a Koszul resolution of $\mathcal{C}_{X}^{\omega}$ we have the standard isomorphism $\mathrm{R} \mathcal{H} \operatorname{lom}_{\rho_{!} \mathcal{D}_{X}}\left(\rho_{!} \mathcal{C}_{X}^{\omega}, \mathcal{C}_{X}^{\infty}\right) \simeq \Omega_{X}^{t}$. In 4 a functor $\mathrm{RTH} H_{X}(F)$ is defined (now denoted $T \mathcal{H} \mathrm{om}\left(F, \mathcal{D} b_{X}\right)$ ) and Proposition 4.6 (loc. cit.) reads

$$
\operatorname{RHom}\left(F, \mathbf{C}_{X}\right) \simeq \operatorname{RHom}_{\mathcal{D}_{X}}\left(\mathcal{C}_{X}^{\omega}, T \mathcal{H} \operatorname{om}\left(F, \mathcal{D} b_{X}\right)\right) .
$$

To replace distributions by $\mathcal{C}^{\infty}$ functions we have an analog of $T \mathcal{H}$ om $\left(F, \mathcal{D} b_{X}\right)$ for $\mathcal{C}^{\infty}$ functions, introduced in [6] and [7]. By [6, Theorem 10.5], we have the comparison isomorphism

$$
\operatorname{RHom}_{\mathcal{D}_{X}}\left(\mathcal{C}_{X}^{\omega}, T \mathcal{H} \operatorname{om}\left(F, \mathcal{C}_{X}^{\infty}\right)\right) \simeq \operatorname{R} \mathcal{H} \operatorname{om}_{\mathcal{D}_{X}}\left(\mathcal{C}_{X}^{\omega}, T \mathcal{H} \operatorname{om}\left(F, \mathcal{D} b_{X}\right)\right)
$$

Actually, in [6] $X$ is a complex manifold and the result is stated for the sheaf of anti-holomorphic functions instead of $\mathcal{C}_{X}^{\omega}$, but the proof also works in our case. By [7, Proposition 7.2.6] or [10, Proposition 3.3.5], we may express the functor $T \mathcal{H}$ om using the subanalytic site: $T \mathcal{H} \operatorname{om}\left(F, \mathcal{C}_{X}^{\infty}\right) \simeq \rho^{-1} \operatorname{RHom}\left(\rho_{*} F, \mathcal{C}_{X}^{\infty}\right)$.

Putting these isomorphisms together we obtain (14):

$$
\begin{aligned}
\operatorname{RHom}\left(\rho_{*} F, \Omega_{X}^{t}\right) & \simeq \operatorname{RHom}\left(\rho_{*} F, \operatorname{R\mathcal {H}} \operatorname{lom}_{\rho ! \mathcal{D}_{X}}\left(\rho_{!} \mathcal{C}_{X}^{\omega}, \mathcal{C}_{X}^{\infty t}\right)\right) \\
& \simeq \operatorname{RHom} \rho_{\rho !} \mathcal{D}_{X}\left(\rho_{!} \mathcal{C}_{X}^{\omega}, \operatorname{R\mathcal {H}}\left(\rho_{*} F, \mathcal{C}_{X}^{\infty t}\right)\right) \\
& \simeq \operatorname{RHom} \mathcal{D}_{X}\left(\mathcal{C}_{X}^{\omega}, T \mathcal{H} \operatorname{om}\left(F, \mathcal{D} b_{X}\right)\right) \\
& \simeq \operatorname{RHom}\left(\rho_{*} F, \mathbf{C}_{X}\right)
\end{aligned}
$$

where we have used adjunction morphisms between $\otimes, \mathcal{H}$ om and $\rho_{!}, \rho^{-1}$. 
The integration of forms also makes sense in the tempered case: we let $f: X \rightarrow Y$ be a submersion with fibers of dimension $d, V \subset Y$ a subanalytic open subset and we consider a form $\omega \in \Gamma\left(f^{-1}(V) ; \Omega_{X}^{t, i+d} \otimes o r_{X \mid Y}\right)$ such that the closure (in $X)$ of $\operatorname{supp} \omega$ is compact. Then $\int_{f} \omega \in \Gamma\left(V ; \Omega_{Y}^{t, i}\right)$. We deduce the morphism of complexes

$$
\int_{f}: f_{! !}\left(\Omega_{X}^{t} \otimes \omega_{X \mid Y}\right) \rightarrow \Omega_{Y}^{t}
$$

Its image in $\mathrm{D}^{b}\left(\mathbf{C}_{Y_{s a}}\right)$ coincides with the morphism $\operatorname{int}_{f}$ of 12 .

\section{§5. Resolution}

In this section all manifolds are real analytic.

Definition 5.1. For a manifold $X$ we introduce the notations $\widehat{X}=X \times \mathbf{R}$, $i_{X}: X \rightarrow \widehat{X}, x \mapsto(x, 0)$, and $X^{+}=X \times \mathbf{R}_{>0}$. We consider the tempered de Rham algebra on the site $\widehat{X}_{s a}$,

$$
\Omega_{\widehat{X}}^{t}=0 \rightarrow \Omega_{\widehat{X}}^{t, 0} \rightarrow \cdots \rightarrow \Omega_{\widehat{X}}^{t, n+1} \rightarrow 0,
$$

and define $\mathcal{A}_{X}=i_{X}^{-1} \Gamma_{X^{+}}\left(\Omega_{\widehat{X}}^{t}\right)$. This is a sheaf of anti-commutative dg-algebras on $X_{s a}$.

The inverse image of forms under the projection $\widehat{X} \rightarrow X$ induces an injective morphism of dg-algebras $\Omega_{X} \hookrightarrow \mathcal{A}_{X}$. In particular $\mathcal{C}_{X}^{\infty, t} \subset \mathcal{A}_{X}^{0}$. We denote by $t$ the coordinate on $\mathbf{R}$. This gives a canonical element $d t \in \mathcal{A}_{X}^{1}$. The decomposition $\widehat{X}=X \times \mathbf{R}$ induces a decomposition of the differential $d=d_{1}+d_{2}$ into anticommuting differentials, where we set $d_{2}(\omega)=(\partial \omega / \partial t) d t$.

The algebra $\mathcal{A}_{X}$ comes equipped with natural morphisms related to inverse image and direct image under a smooth map. Let $f: X \rightarrow Y$ be a morphism of manifolds. It induces $\widehat{f}=f \times$ id: $\widehat{X} \rightarrow \widehat{Y}$ and $f^{+}: X^{+} \rightarrow Y^{+}$. We consider the morphism of functors $\widehat{f}^{-1} \Gamma_{Y^{+}} \rightarrow \Gamma_{X^{+}} \widehat{f}^{-1}$; it induces a morphism of dg-algebras

$$
\widehat{f}^{-1} \Gamma_{Y^{+}}\left(\Omega_{\widehat{Y}}^{t}\right) \rightarrow \Gamma_{X^{+}} \widehat{f}^{-1}\left(\Omega_{\widehat{Y}}^{t}\right) \rightarrow \Gamma_{X^{+}}\left(\Omega_{\widehat{X}}^{t}\right) .
$$

Definition 5.2. We denote by $f^{\sharp}: f^{-1} \mathcal{A}_{Y} \rightarrow \mathcal{A}_{X}$ the image of the above morphism under the restriction functor $i_{X}^{-1}$. It is a morphism of dg-algebras.

Now we assume that $f$ is smooth. Hence $\widehat{f}$ is also smooth and we have the integration morphism 15$) \int_{\widehat{f}}: \widehat{f}_{! !}\left(\Omega_{\widehat{X}}^{t} \otimes \omega_{X \mid Y}\right) \rightarrow \Omega_{\widehat{Y}}^{t}$. We apply the functor $i_{Y}^{-1} \Gamma_{Y^{+}}$ to this morphism. We also have the base change $f_{! !} i_{X}^{-1} \simeq i_{Y}^{-1} \widehat{f}_{! !}$and the morphism 
$\widehat{f}_{! !} \Gamma_{X^{+}} \rightarrow \Gamma_{Y^{+}} \widehat{f}_{! !}$. They give the sequence of morphisms

$$
f_{! !} i_{X}^{-1} \Gamma_{X^{+}}\left(\Omega_{\widehat{X}}^{t} \otimes \omega_{\widehat{X} \mid \widehat{Y}}\right) \rightarrow i_{Y}^{-1} \Gamma_{Y^{+}} \widehat{f}_{! !}\left(\Omega_{\widehat{X}}^{t} \otimes \omega_{\widehat{X} \mid \widehat{Y}}\right) \rightarrow i_{Y}^{-1} \Gamma_{Y^{+}} \Omega_{\widehat{Y}}^{t} .
$$

Definition 5.3. For a smooth map $f: X \rightarrow Y$, we call morphism 116) the integration morphism and denote it $\int_{f}: f_{! !}\left(\mathcal{A}_{X} \otimes \omega_{X \mid Y}\right) \rightarrow \mathcal{A}_{Y}$.

The main result of this section is the following theorem. It is proved in the remaining part of the section: the quasi-injectivity of the $\mathcal{A}_{X}^{i}$ is proved in Proposition 5.11, and the fact that $\mathcal{A}_{X}$ is a resolution is Corollary 5.10.

Theorem 5.4. Let $X$ be a real analytic manifold. The sheaf of dg-algebras $\mathcal{A}_{X}$ is a quasi-injective resolution of $\mathbf{C}_{X_{s a}}$.

Remark 5.5. By this theorem we have $f_{! !}\left(\mathcal{A}_{X} \otimes \omega_{X \mid Y}\right) \simeq \mathrm{R} f_{! !}\left(\omega_{X \mid Y}\right)$. Hence the morphism $\int_{f}$ of Definition 5.3 induces a morphism in the derived category $\mathrm{R} f ! ! \omega_{X \mid Y} \rightarrow \mathbf{C}_{Y_{s a}}$. It coincides with the topological integration morphism 12 because this holds for the usual de Rham resolution.

For the proof of the theorem we need some lemmas on tempered functions. We refer to [3] for results on subanalytic sets. We recall that a function is subanalytic if its graph is a subanalytic set. We introduce the following notation, for $U \subset X$ an open subset and $\varphi: U \rightarrow \mathbf{R}$ a positive continuous function:

$$
U_{\varphi}=\{(x, t) \in \widehat{X} ; x \in U,|t|<\varphi(x)\}, \quad U_{\varphi}^{+}=U_{\varphi} \cap X^{+} .
$$

Lemma 5.6. Let $U \subset X$ be a subanalytic open subset and $V \subset \widehat{X}$ be a subanalytic open neighborhood of $U$ in $\widehat{X}$. Then there exists a subanalytic continuous function $\varphi$ defined on $\bar{U}$ such that $\varphi=0$ on $\partial U$ and $U_{\varphi} \subset V$.

Proof. We set $V^{\prime}=V \cap(U \times \mathbf{R}), Z=\widehat{X} \backslash V^{\prime}$ and let $\varphi$ be the distance function to $Z: \varphi(x)=d(x, Z)$. By [3, Remark 3.11], this is a subanalytic function on $\widehat{X}$ and its restriction to $\bar{U}$ satisfies the required property.

The following result is similar to a division property for flat $\mathcal{C}^{\infty}$ functions, which can be found for example in [13, Lemma V.2.4].

Lemma 5.7. Let $U \subset X$ be a subanalytic open subset and $\varphi: \bar{U} \rightarrow \mathbf{R}$ a subanalytic continuous function on $U$, such that $\varphi=0$ on the boundary of $U$ and $\varphi>0$ on $U$. Then there exist another subanalytic continuous function $\varphi^{\prime}$ on $U$ and a $\mathcal{C}^{\infty}$ function $\psi: U \rightarrow \mathbf{R}$ such that
(i) $\forall x \in U, 0<\varphi^{\prime}(x)<\psi(x)<\varphi(x)$,
(ii) $\psi$ and $1 / \psi$ are tempered. 
Proof. (a) We first note that (i) and " $\psi$ tempered" imply that $1 / \psi$ is tempered. Indeed it is enough to check that $1 / \psi$ has polynomial growth along $\partial U$. But this follows from Łojasiewicz's inequality (see [3, Theorem 6.4 and Remark 6.5]) applied to $\varphi^{\prime}$. We may also work locally: assuming the result is true in local charts, we choose

- locally finite coverings of $X$ by subanalytic open subsets, $\left(U_{i}\right),\left(V_{i}\right)$, together with a partition of unity $\mu_{i}: X \rightarrow \mathbf{R}$ and subanalytic continuous functions $\nu_{i}: X \rightarrow \mathbf{R}$ such that $\bar{U}_{i} \subset V_{i}, 0 \leq \nu_{i} \leq \mu_{i}, \sum \mu_{i}=1, \mu_{i}=\nu_{i}=1$ on $U_{i}$ and $\mu_{i}=0$ on a neighborhood of $X \backslash V_{i}$,

- $\mathcal{C}^{\infty}$ tempered functions $\psi_{i}: U \cap V_{i} \rightarrow \mathbf{R}$ and subanalytic continuous functions $\varphi_{i}^{\prime}: U \cap V_{i} \rightarrow \mathbf{R}$ such that $0<\varphi_{i}^{\prime}<\psi_{i}<\varphi$ on $U \cap V_{i}$,

and we set $\psi=\sum_{i} \mu_{i} \psi_{i}, \varphi^{\prime}=\sum_{i} \nu_{i} \varphi_{i}^{\prime}$. Then $\psi, \varphi^{\prime}$ satisfy the conclusion of the lemma. Indeed, each $\mu_{i} \psi_{i}$ is defined and tempered on $U$ and their sum is locally finite, hence also tempered.

(b) Hence we assume $X=\mathbf{R}^{n}$ and $U$ is bounded. By [13, Lemma IV.3.3, there exist constants $C_{k}, k \in \mathbf{N}^{n}$, such that, for any compact $K \subset \mathbf{R}^{n}$ and any $\varepsilon>0$, there exists a $\mathcal{C}^{\infty}$ function $\alpha$ on $\mathbf{R}^{n}$ such that

$$
\begin{aligned}
0 \leq \alpha \leq 1, \quad \alpha(x)=0 \text { if } d(x, K) & \geq \varepsilon, \quad \alpha(x)=1 \text { if } x \in K, \\
\forall k \in \mathbf{N}^{n}, \quad\left|D^{k} \alpha\right| & \leq C_{k} \varepsilon^{-|k| .}
\end{aligned}
$$

(The function $\alpha$ is the convolution of the characteristic function of $\{x ; d(x, K)$ $\leq \varepsilon / 2\}$ with a suitable test function.)

We set $K_{i}=\left\{x \in U ; 2^{-i-1} \leq d(x, \partial U) \leq 2^{-i}\right\}$ and we let $\alpha_{i}$ be a function associated to $K=K_{i}$ and $\varepsilon=2^{-i-2}$ by the above result. In particular $\alpha_{i}=1$ on $K_{i}$, supp $\alpha_{i} \subset S_{i}$, where we set $S_{i}=K_{i-1} \cup K_{i} \cup K_{i+1}$, and $\left|D^{k} \alpha_{i}\right| \leq C_{k}^{\prime} 2^{i k}$ for some $C_{k}^{\prime} \in \mathbf{R}$. This implies that for $x \in U,\left|D^{k} \alpha_{i}(x)\right| \leq C_{k}^{\prime \prime} d(x, \partial U)^{-k}$ for some other constants $C_{k}^{\prime \prime} \in \mathbf{R}$.

Lojasiewicz's inequality gives, for $x \in U, c d(x, \partial U)^{r} \leq \varphi(x) \leq c^{\prime} d(x, \partial U)^{r^{\prime}}$ for some $c, r, c^{\prime}, r^{\prime}>0$ (see [3, Theorem 6.4]). We set $\lambda_{i}=\min \left\{\varphi(x) ; x \in S_{i}\right\}$. We note that for $x, x^{\prime} \in S_{i}$, we have $1 / 8 \leq d(x, \partial U) / d\left(x^{\prime}, \partial U\right) \leq 8$. Hence, for $x \in S_{i}$, we have $C d(x, \partial U)^{r} \leq \lambda_{i} \leq C^{\prime} d(x, \partial U)^{r^{\prime}}$ for some $C, C^{\prime}>0$. Since $\operatorname{supp} \alpha_{i} \subset S_{i}$, we also have $\lambda_{i} \alpha_{i} \leq \varphi$ for all $i$.

We note that each $x \in U$ belongs to at most three sets $S_{i}$ and we define $\psi=(1 / 3) \sum_{i} \lambda_{i} \alpha_{i}$. The above inequalities give, for $x \in U, 0<\psi(x) \leq \varphi(x)$ and

$$
\left|D^{k} \psi(x)\right| \leq C_{k}^{\prime \prime} C^{\prime} d(x, \partial U)^{r^{\prime}-k}, \quad \frac{1}{\psi(x)} \leq 3 C^{-1} d(x, \partial U)^{-r} .
$$

Hence $\psi$ is tempered and we can take $\varphi^{\prime}(x)=(C / 4) d(x, \partial U)^{r}$. 
Lemma 5.8. Let $U$ be an open subanalytic subset of $X$ and $Z_{1}, Z_{2}$ disjoint closed subanalytic subsets of $U$. There exists a tempered function $\alpha \in \Gamma\left(U ; \mathcal{C}_{X}^{\infty, t}\right)$ such that $0 \leq \alpha \leq 1, \alpha=1$ on $Z_{1}$ and $\alpha=0$ on $Z_{2}$.

Proof. We set $U_{i}=U \backslash Z_{i}, i=1,2$, and define the subanalytic function $\varphi_{i}$ on $\overline{U_{i}}$ by $\varphi_{i}(x)=d\left(x, \partial U_{i}\right)$. We apply Lemma 5.7 to $U_{i}$ and $\varphi_{i}$ to obtain subanalytic functions $\varphi_{i}^{\prime}$ and $\mathcal{C}^{\infty}$ functions $\psi_{i}$ on $U_{i}$ satisfying the conclusions of the lemma. We define functions on $V=U \backslash\left(Z_{1} \cup Z_{2}\right), r=\psi_{1} / \psi_{2}, s=\varphi_{1}^{\prime} / \varphi_{2}, s^{\prime}=\varphi_{1} / \varphi_{2}^{\prime}$ so that $r$ is $\mathcal{C}^{\infty}, s, s^{\prime}$ are subanalytic and we have the inequalities $0<s<r<s^{\prime}$. We also note that $r$ is tempered on $V$.

We set $V_{1}=Z_{1} \cup\left\{s^{\prime}<1\right\}, V_{2}=Z_{2} \cup\{2<s\}$. These are subanalytic open subsets of $X$ and $U=V \cup V_{1} \cup V_{2}$. We choose a $\mathcal{C}^{\infty}$ function $h$ on $\mathbf{R}$ such that $0 \leq h \leq 1, h(t)=1$ for $t \leq 1$ and $h(t)=0$ for $t \geq 2$. Now we define $\alpha$ by $\alpha=1$ on $V_{1}, \alpha=0$ on $V_{2}$ and $\alpha=h \circ r$ on $V$. We see that $\alpha$ is well-defined and $\mathcal{C}^{\infty}$ on $U$. It is clearly tempered on $V_{1}$ and $V_{2}$. Since the derivatives of $h$ (to a given order) can be uniformly bounded on $\mathbf{R}$ and $r$ is tempered on $V$ we see that $h \circ r$ is also tempered on $V$. Hence $\alpha$ is tempered on $U$ as required.

Proposition 5.9. Any $\mathcal{C}_{X}^{\infty, t}$-module or $\mathcal{A}_{X}^{0}$-module is soft in the sense of Definition 4.1 .

Proof. Because of the inclusion $\mathcal{C}_{X}^{\infty, t} \subset \mathcal{A}_{X}^{0}$ it is enough to prove the result for every $\mathcal{C}_{X}^{\infty, t}$-module $F$.

Let $U$ and $Z$ be respectively open and closed subanalytic subsets of $X$ and consider $s \in \Gamma\left(U ; F_{Z}\right)$. We may assume $s \in \Gamma(W ; F)$ for a subanalytic open set $W$ with $U \cap Z \subset W \subset U$. We choose two subanalytic open sets $W_{1}, W_{2}$ such that $U \cap Z \subset W_{1} \subset U \cap \overline{W_{1}} \subset W_{2} \subset U \cap \overline{W_{2}} \subset W$. By Lemma 5.8 we may find $\alpha \in \Gamma\left(U ; \mathcal{C}_{X}^{\infty, t}\right)$ such that $\alpha=1$ on $\overline{W_{1}}$ and $\alpha=0$ on $U \backslash W_{2}$. Then $\alpha s \in \Gamma(W ; F)$ extends by 0 on $U$ and $\alpha s=s$ in $\Gamma\left(U ; F_{Z}\right)$. It follows that $\Gamma(U ; F) \rightarrow \Gamma\left(U ; F_{Z}\right)$ is surjective, as required.

Corollary 5.10. The sheaf of dg-algebras $\mathcal{A}_{X}$ is a resolution of $\mathbf{C}_{X_{\text {sa }}}$, i.e. we have the exact sequence $0 \rightarrow \mathbf{C}_{X_{s a}} \rightarrow \mathcal{A}_{X}^{0} \rightarrow \mathcal{A}_{X}^{1} \rightarrow \cdots \rightarrow \mathcal{A}_{X}^{n+1} \rightarrow 0$.

Proof. The sequence is obtained from the exact sequence of Lemma 4.7 (on $\widehat{X}$ ) by applying the functors $\Gamma_{X^{+}}$and $i_{X}^{-1}$. Since $i_{X}^{-1}$ is exact, it just remains to see that the terms $\mathbf{C}_{\widehat{X}_{s a}}$ and $\Omega_{\widehat{X}}^{t, i}$ are $\Gamma_{X^{+}-\text {acyclic. }}$

For $\Omega_{\hat{X}}^{t, i}$ this follows from Proposition 5.9 and Corollary 4.5 For $\mathbf{C}_{\widehat{X}_{s a}}$ it follows from $\mathrm{R}_{X^{+}}\left(\mathbf{C}_{\widehat{X}_{s a}}\right) \simeq \mathbf{C}_{X_{s a}^{+}}$(recall that $\rho_{*}$ commutes with $\left.\mathrm{R} \Gamma_{X^{+}}\right)$. 
Proposition 5.11. Let $F$ be a $\mathcal{C}_{\widehat{X}}^{\infty, t}{ }^{\infty}$ module and set $G=i_{X}^{-1} \Gamma_{X+} F$. Let $U \subset X$ be a subanalytic open subset. Then the natural map $\Gamma\left(X^{+} ; F\right) \rightarrow \Gamma(U ; G)$ is surjective and $G$ is quasi-injective. In particular the $\mathcal{A}_{X}^{i}$ are quasi-injective.

Proof. We consider $s \in \Gamma(U ; G)$. As in the case of sheaves on manifolds we have, for $H \in \operatorname{Mod}\left(\mathbf{C}_{\widehat{X}_{s a}}\right)$ and $U \subset X, \Gamma\left(U ; i_{X}^{-1} H\right) \simeq \lim _{\longrightarrow} \Gamma(V ; H)$ where $V$ runs over the subanalytic open subsets of $\widehat{X}$ containing $U$. By Lemma 5.6 , we may assume $V=U_{\varphi}^{+}$for some $\varphi: \bar{U} \rightarrow \mathbf{R}$, so that $s$ is represented by a section $\tilde{s} \in \Gamma\left(U_{\varphi}^{+} ; F\right)$. Since $X^{+} \cap \overline{U_{\varphi / 2}^{+}} \subset U_{\varphi}^{+}$this $\tilde{s}$ defines a section of $\Gamma\left(X^{+} ; F_{\overline{U_{\varphi / 2}^{+}}}\right)$. By Proposition 5.9 we may extend it to $\hat{s} \in \Gamma\left(X^{+} ; F\right)$ and we have $\left.\hat{s}\right|_{U_{\varphi / 2}^{+}}=\left.\tilde{s}\right|_{U_{\varphi / 2}^{+}}$. This shows the surjectivity of $\Gamma\left(X^{+} ; F\right) \rightarrow \Gamma(U ; G)$. Since this morphism factors through $\Gamma(X ; G) \rightarrow \Gamma(U ; G)$ this also proves that $G$ is quasi-injective.

\section{§6. $\mathcal{A}$-modules}

For a real analytic manifold $X$, we denote by $\operatorname{Mod}\left(\mathcal{A}_{X}\right)$ the category of sheaves of $\operatorname{dg}-\mathcal{A}_{X}$-modules bounded below on $X_{s a}$. We have an obvious forgetful functor and its composition with the localization:

$$
\operatorname{For}_{X}: \operatorname{Mod}\left(\mathcal{A}_{X}\right) \rightarrow \mathrm{C}^{+}\left(\mathbf{C}_{X_{s a}}\right), \quad \operatorname{For}_{X}^{\prime}: \operatorname{Mod}\left(\mathcal{A}_{X}\right) \rightarrow \mathrm{D}^{+}\left(\mathbf{C}_{X_{s a}}\right) .
$$

We will usually write $F$ instead of $\operatorname{For}_{X}(F)$ or $\operatorname{For}_{X}^{\prime}(F)$ when the context is clear. We still write For $_{X}$, For $_{X}^{\prime}$ for the compositions of these forgetful functors with the exact functor $I_{\tau}: \mathrm{C}\left(\mathbf{C}_{X_{s a}}\right) \rightarrow \mathrm{C}\left(\mathbf{I}\left(\mathbf{C}_{X}\right)\right)$.

In this section we define operations on $\operatorname{Mod}\left(\mathcal{A}_{X}\right)$ and check usual formulas in this framework, as well as some compatibility with the corresponding operations in $\mathrm{C}\left(\mathbf{C}_{X_{s a}}\right)$ or $\mathrm{D}\left(\mathbf{C}_{X_{s a}}\right)$ (hence also in $\mathrm{C}\left(\mathbf{I}\left(\mathbf{C}_{X}\right)\right)$ or $\mathrm{D}\left(\mathbf{I}\left(\mathbf{C}_{X}\right)\right)$, because $I_{\tau}$ commutes with the standard operations).

Tensor product. For $\mathcal{M}, \mathcal{N} \in \operatorname{Mod}\left(\mathcal{A}_{X}\right)$, the tensor product $\mathcal{M} \otimes_{\mathcal{A}_{X}} \mathcal{N} \in$ $\operatorname{Mod}\left(\mathcal{A}_{X}\right)$ is defined as usual by taking the tensor product of the underlying sheaves of graded modules over the underlying sheaf of graded algebras and defining the differential by $d(m \otimes n)=d m \otimes n+(-1)^{\operatorname{deg} m} m \otimes d n$ (for $m$ homogeneous). We have an exact sequence in $\mathrm{C}^{+}\left(\mathbf{C}_{X_{s a}}\right)$,

$$
\mathcal{M} \otimes \mathcal{A}_{X} \otimes \mathcal{N} \stackrel{\delta}{\rightarrow} \mathcal{M} \otimes \mathcal{N} \rightarrow \mathcal{M} \otimes_{\mathcal{A}_{X}} \mathcal{N} \rightarrow 0
$$

where $\delta(m \otimes a \otimes n)=(-1)^{\operatorname{deg} a \operatorname{deg} m} a m \otimes n-m \otimes a n$ for $a, m, n$ homogeneous. For two real analytic manifolds $X, Y$ and $\mathcal{M} \in \operatorname{Mod}\left(\mathcal{A}_{X}\right), \mathcal{N} \in \operatorname{Mod}\left(\mathcal{A}_{Y}\right)$, we denote by $\underline{\otimes}$ the external tensor product in the category of $\mathcal{A}$-modules,

$$
\mathcal{M} \unrhd \mathcal{N}=\mathcal{A}_{X \times Y} \otimes_{\left(\mathcal{A}_{X} \otimes \mathcal{A}_{Y}\right)}(\mathcal{M} \otimes \mathcal{N}) .
$$


Proposition 6.1. Let $I, F \in \operatorname{Mod}\left(\mathcal{A}_{X}\right)$ be such that $I$ is quasi-injective and $F$ has a finite presentation $\left(\mathcal{A}_{X}\right)^{N} \rightarrow F \rightarrow 0$. Then $I \otimes_{\mathcal{A}_{X}} F$ is quasi-injective.

Proof. Let $U \subset X$ be a subanalytic open subset. The morphism $u: I^{N} \rightarrow I \otimes_{\mathcal{A}_{X}} F$ is surjective. Since ker $u$ is an $\mathcal{A}_{X}^{0}$-module, Proposition 5.9 and Corollary 4.5 imply that it is acyclic for $\Gamma(U ; \cdot)$. It follows that the morphism $\Gamma\left(U ; I^{N}\right)$ $\rightarrow \Gamma\left(U ; I \otimes_{\mathcal{A}_{X}} F\right)$ is surjective. Since $I^{N}$ is quasi-injective this implies that $\Gamma\left(X ; I \otimes_{\mathcal{A}_{X}} F\right) \rightarrow \Gamma\left(U ; I \otimes_{\mathcal{A}_{X}} F\right)$ is surjective too, which proves the result.

Inverse image and direct image. Let $f: X \rightarrow Y$ be a morphism of real analytic manifolds. Recall the morphism $f^{\sharp}: f^{-1} \mathcal{A}_{Y} \rightarrow \mathcal{A}_{X}$ of Definition 5.2. For $\mathcal{N} \in$ $\operatorname{Mod}\left(\mathcal{A}_{Y}\right)$ we define its inverse image in $\operatorname{Mod}\left(\mathcal{A}_{X}\right)$,

$$
f^{*} \mathcal{N}=\mathcal{A}_{X} \otimes_{f^{-1} \mathcal{A}_{Y}} f^{-1} \mathcal{N}
$$

By adjunction $f^{\sharp}$ gives a morphism $\mathcal{A}_{Y} \rightarrow f_{*} \mathcal{A}_{X}$. Hence, for $\mathcal{M} \in \operatorname{Mod}\left(\mathcal{A}_{X}\right), f_{*} \mathcal{M}$ has a natural structure of $\mathrm{dg}-\mathcal{A}_{Y}$-module, as also has $f_{! !} \mathcal{M}$, through the natural morphism $f_{*} \mathcal{A}_{X} \otimes f_{! !} \mathcal{M} \rightarrow f_{! !}\left(\mathcal{A}_{X} \otimes \mathcal{M}\right) \rightarrow f_{! !} \mathcal{M}$.

We have a natural morphism $f^{-1} \mathcal{N} \rightarrow f^{*} \mathcal{N}$ in $\mathrm{C}\left(\mathbf{C}_{X_{s a}}\right)$ (with the notations of (17), we could write more precisely $f^{-1}\left(\operatorname{For}_{Y} \mathcal{N}\right) \rightarrow$ For $\left._{X} f^{*} \mathcal{N}\right)$. We show in Proposition 6.3 that it is a quasi-isomorphism when $f$ is smooth. We first consider a particular case.

Lemma 6.2. Set $X=\mathbf{R}^{m+1}, Y=\mathbf{R}^{m}$ and let $f: X \rightarrow Y$ be the projection. Consider coordinates $\left(y_{1}, \ldots, y_{m}, u\right)$ on $X$. For $\mathcal{N} \in \operatorname{Mod}\left(\mathcal{A}_{Y}^{0}\right)$ we have an exact sequence in $\operatorname{Mod}\left(\mathbf{C}_{X_{s a}}\right)$,

$$
0 \rightarrow f^{-1} \mathcal{N} \rightarrow \mathcal{A}_{X}^{0} \otimes_{f^{-1}} \mathcal{A}_{Y}^{0} f^{-1} \mathcal{N} \stackrel{d}{\rightarrow} \mathcal{A}_{X}^{0} \otimes_{f^{-1}} \mathcal{A}_{Y}^{0} f^{-1} \mathcal{N} \rightarrow 0,
$$

where $d$ is defined by $d(a \otimes n)=\frac{\partial a}{\partial u} \otimes n$ for $a \in \mathcal{A}_{X}^{0}, n \in \mathcal{N}$.

Proof. We have the exact sequence $0 \rightarrow f^{-1} \mathcal{A}_{Y}^{0} \rightarrow \mathcal{A}_{X}^{0} \stackrel{d}{\rightarrow} \mathcal{A}_{X}^{0} \rightarrow 0$ where $d(a)=\frac{\partial a}{\partial u}$. The tensor product with $f^{-1} \mathcal{N}$ gives the exactness of the sequence of the lemma except at the first term. It just remains to check that $\iota: f^{-1} \mathcal{N} \rightarrow$ $\mathcal{A}_{X}^{0} \otimes_{f^{-1} \mathcal{A}_{Y}^{0}} f^{-1} \mathcal{N}, n \mapsto 1 \otimes n$, is injective.

(a) We consider a section $n \in \Gamma\left(U ; f^{-1} \mathcal{N}\right)$ such that $\iota(n)=0$. This means that there exist a locally finite covering $U=\bigcup_{i \in I} U_{i}$ and, setting $V_{i}=f\left(U_{i}\right)$, sections $n_{i}, n_{i j} \in \Gamma\left(V_{i} ; \mathcal{N}\right), a_{i j} \in \Gamma\left(U_{i} ; \mathcal{A}_{X}^{0}\right), b_{i j} \in \Gamma\left(V_{i} ; \mathcal{A}_{Y}^{0}\right)$, where $j$ runs over a finite set $J_{i}$, such that for each $i \in I,\left.n\right|_{U_{i}}=f^{*} n_{i}$ and we have the identity in $\Gamma\left(U_{i} ; \mathcal{A}_{X}^{0}\right) \otimes \Gamma\left(V_{i} ; \mathcal{N}\right)$

$$
1 \otimes n_{i}=\sum_{j \in J_{i}}\left(a_{i j}\left(b_{i j} \circ f\right) \otimes n_{i j}-a_{i j} \otimes b_{i j} n_{i j}\right) .
$$


We may as well assume that the $\overline{U_{i}}$ are compact. We show in this case that $n_{i}=0$, which will prove $n=0$, hence the injectivity of $\iota$.

(b) By Proposition 5.11 we may represent the $a_{i j}, b_{i j}$ by tempered $\mathcal{C}^{\infty}$ functions defined on $X^{+}, Y^{+}$. We choose continuous subanalytic functions $\varphi_{i}: \overline{U_{i}} \rightarrow \mathbf{R}$, $\varphi_{i}>0$ on $U_{i}$, such that the identities 19 hold in $\Gamma\left(\left(U_{i}\right)_{\varphi_{i}}^{+} ; \mathcal{C}_{\widehat{X}}^{\infty, t}\right) \otimes \Gamma\left(V_{i} ; \mathcal{N}\right)$.

By Lemma 5.8 we may choose $\alpha_{i} \in \Gamma\left(X^{+} ; \mathcal{C}_{\widehat{X}}^{\infty, t}\right)$ such that $0 \leq \alpha_{i} \leq 1, \alpha_{i}=1$ on $\left(U_{i}\right)_{\varphi_{i} / 4}^{+}$and $\alpha_{i}=0$ outside $\left(U_{i}\right)_{\varphi_{i} / 2}^{+}$. Multiplying both sides of 19 by $\alpha_{i}$ we obtain identities which now hold on $\Gamma\left(X^{+} ; \mathcal{C}_{\widehat{X}}^{\infty, t}\right) \otimes \Gamma\left(V_{i} ; \mathcal{N}\right)$. These identities imply

$$
\alpha_{i} \otimes n_{i}=0 \quad \text { in } \quad \Gamma\left(X^{+} ; \mathcal{C}_{\widehat{X}}^{\infty, t}\right) \otimes_{\Gamma\left(Y^{+} ; \mathcal{C}_{\widehat{Y}}^{\infty, t}\right)} \Gamma\left(V_{i} ; \mathcal{N}\right)
$$

We note that $\alpha_{i}$ has compact support and we set $\beta_{i}=\int_{\hat{f}} \alpha_{i} d u$. We have $\beta_{i} \in$ $\Gamma\left(Y^{+} ; \mathcal{C}_{\widehat{Y}}^{\infty, t}\right)$ and the last identity gives $\beta_{i} n_{i}=0$. Now $\Gamma\left(V_{i} ; \mathcal{N}\right)$ is a $\Gamma\left(V_{i} ; \mathcal{A}_{Y}^{0}\right)$ module and to conclude that $n_{i}=0$ it just remains to prove that $\left.\beta_{i}\right|_{V_{i}}$ is invertible in $\Gamma\left(V_{i} ; \mathcal{A}_{Y}^{0}\right)$.

(c) Since $\beta_{i}$ is a tempered $\mathcal{C}^{\infty}$ function on $Y^{+}$it is enough to check that $\beta_{i}^{-1}$ has polynomial growth along the boundary of $W_{i}=f\left(\left(U_{i}\right)_{\varphi_{i} / 4}^{+}\right)$. We set $Z_{i}=X^{+} \backslash\left(U_{i}\right)_{\varphi_{i} / 4}^{+}$and for $(x, t) \in X^{+}, d_{i}(x, t)=d\left((x, t), \partial Z_{i}\right)$. We obtain the bound, for $(y, t) \in W_{i}$,

$$
\beta_{i}(y, t) \geq \int_{\left(U_{i}\right)_{\varphi_{i} / 4}^{+} \cap(\{(y, t)\} \times \mathbf{R})} 1 \cdot d u \geq 2 \max _{u \in \mathbf{R}} d_{i}(y, u, t) .
$$

The function $m_{i}(y, t)=\max _{u \in \mathbf{R}} d_{i}(y, u, t)$ is subanalytic since the max can be taken for $u$ running over a compact set. We have $m_{i}(y, t)>0$ for $(y, t) \in W_{i}$. Hence, by Łojasiewicz's inequality we have $m_{i}(y, t)>C d\left((y, t), \partial W_{i}\right)^{-N}$ for some $C, N \in \mathbf{R}$ and it follows that $\beta_{i}^{-1}$ has polynomial growth along $\partial W_{i}$.

Proposition 6.3. Let $f: X \rightarrow Y$ be a smooth morphism and $\mathcal{N} \in \operatorname{Mod}\left(\mathcal{A}_{Y}\right)$.

(i) The morphism in $\mathrm{C}\left(\mathbf{C}_{X_{s a}}\right), f^{-1} \mathcal{N} \rightarrow f^{*} \mathcal{N}$, is a quasi-isomorphism.

(ii) If $\mathcal{N}$ is locally free as an $\mathcal{A}_{Y}^{0}$-module, then $f^{*} \mathcal{N}$ is locally free as an $\mathcal{A}_{X}^{0}$ module.

(iii) If $\mathcal{N}$ is flat over $\mathcal{A}_{Y}^{0}$ and we have an exact sequence in $\operatorname{Mod}\left(\mathcal{A}_{Y}\right), 0 \rightarrow \mathcal{N}^{\prime \prime} \rightarrow$ $\mathcal{N}^{\prime} \rightarrow \mathcal{N} \rightarrow 0$, then the sequence $0 \rightarrow f^{*} \mathcal{N}^{\prime \prime} \rightarrow f^{*} \mathcal{N}^{\prime} \rightarrow f^{*} \mathcal{N} \rightarrow 0$ is exact.

Proof. The statements are local on $X$ so that, up to restriction to open subsets, we may assume $X=Y \times \mathbf{R}^{n}$ and $f$ is the projection. Then we factorize $f$ as a composition of projections with fiber dimension 1, so that we may even assume $X=Y \times \mathbf{R}$ (and $\widehat{X}=Y \times \mathbf{R} \times \mathbf{R})$. We take coordinates $\left(y_{1}, \ldots, y_{m}, u, t\right)$ on $\widehat{X}$ ( $u$ is the coordinate in the fiber of $\hat{f}$ ). 
With this decomposition of $X$ we define the $\mathcal{A}_{X}^{0}$-module $\mathcal{A}_{\text {vert }}=\mathcal{A}_{X}^{0} \oplus \mathcal{A}_{X}^{0} d u$. This is a sub- $\mathcal{A}_{X}^{0}$-algebra of $\mathcal{A}_{X}$ (not a sub-dg-algebra); $f^{-1} \mathcal{A}_{Y}$ is another subalgebra, and the multiplication $\mathcal{A}_{\text {vert }} \otimes_{f^{-1} \mathcal{A}_{Y}^{0}} f^{-1} \mathcal{A}_{Y} \rightarrow \mathcal{A}_{X}$, is an isomorphism of $\mathcal{A}_{X}^{0}$-algebras. This shows that for any $\operatorname{dg}-\mathcal{A}_{Y}$-module $\mathcal{N}^{\prime}$ we have an isomorphism of $\mathcal{A}_{X}^{0}$-modules

$$
\mathcal{A}_{\text {vert }} \otimes_{f^{-1} \mathcal{A}_{Y}^{0}} f^{-1} \mathcal{N}^{\prime} \stackrel{\sim}{\rightarrow} f^{*} \mathcal{N}^{\prime} .
$$

Assertions (ii) and (iii) follow easily. Now we prove (i). By 20 again, $f^{*} \mathcal{N}$ is identified with the total complex of the double complex with two rows:

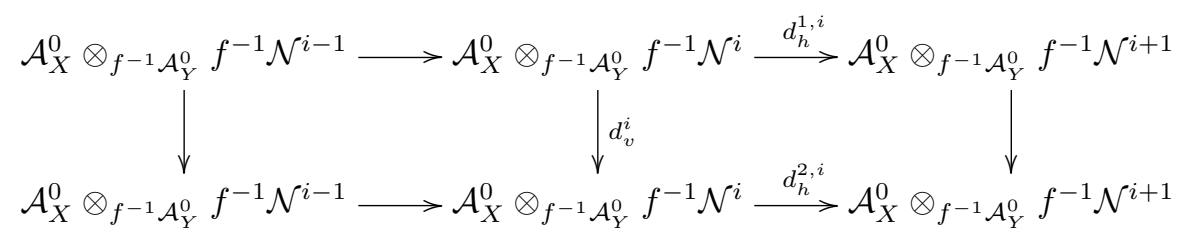

where $d_{v}^{i}(a \otimes n)=\frac{\partial a}{\partial u} \otimes n, \quad d_{h}^{1, i}(a \otimes n)=\sum_{k} \frac{\partial a}{\partial y_{k}} \otimes d y_{k} \cdot n+\frac{\partial a}{\partial t} \otimes d t \cdot n$ and $d_{h}^{2, i}=-d_{h}^{1, i}$. By Lemma 6.2 the $i^{\text {th }}$ column is a resolution of $f^{-1} \mathcal{N}^{i}$. The induced differential on the cohomology of the columns is easily seen to be the differential of $f^{-1} \mathcal{N}$ and (i) follows.

Remark 6.4. Statement (iii) of Proposition 6.3 could be improved if we knew that $\mathcal{A}_{X}^{0}$ is flat over $f^{-1} \mathcal{A}_{Y}^{0}$ but the author does not know whether this is true.

Proposition 6.5. Let $f: X \rightarrow Y$ be a morphism of real analytic manifolds. For any $\mathcal{M} \in \operatorname{Mod}\left(\mathcal{A}_{X}\right)$ we have isomorphisms in $\mathrm{D}^{+}\left(\mathbf{C}_{Y_{s a}}\right)$,

$$
\operatorname{For}^{\prime}\left(f_{*}(\mathcal{M})\right) \simeq \mathrm{R} f_{*}\left(\operatorname{For}^{\prime}(\mathcal{M})\right), \quad \operatorname{For}^{\prime}\left(f_{! !}(\mathcal{M})\right) \simeq \mathrm{R} f_{! !}\left(\operatorname{For}^{\prime}(\mathcal{M})\right) .
$$

Proof. By Proposition 5.9. For $(\mathcal{M}) \in \mathrm{C}^{+}\left(\mathbf{C}_{X_{s a}}\right)$ consists of soft sheaves. Hence Corollary 4.5 gives the result.

\section{Projection formula}

Lemma 6.6. Let $f: X \rightarrow Y$ be a morphism of analytic manifolds, $\mathcal{M} \in \operatorname{Mod}\left(\mathcal{A}_{X}\right)$, $\mathcal{N} \in \operatorname{Mod}\left(\mathcal{A}_{Y}\right)$. There exists a natural isomorphism in $\operatorname{Mod}\left(\mathcal{A}_{Y}\right)$,

$$
\mathcal{N} \otimes_{\mathcal{A}_{Y}} f_{! !} \mathcal{M} \stackrel{\sim}{\rightarrow} f_{! !}\left(f^{*} \mathcal{N} \otimes_{\mathcal{A}_{X}} \mathcal{M}\right)
$$

whose image in $\mathrm{C}^{+}\left(\mathbf{C}_{Y_{\text {sa }}}\right)$ gives a commutative diagram

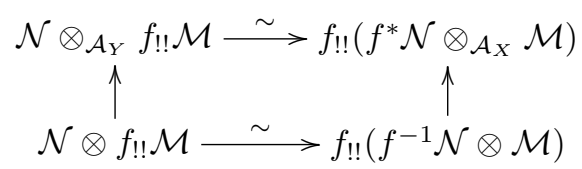

where the bottom arrow is the usual projection formula. 
Proof. Using (18) and $f^{*} \mathcal{N} \otimes_{\mathcal{A}_{X}} \mathcal{M} \simeq f^{-1} \mathcal{N} \otimes_{f^{-1} \mathcal{A}_{Y}} \mathcal{M}$ we have the commutative diagram (extending the diagram of the lemma)

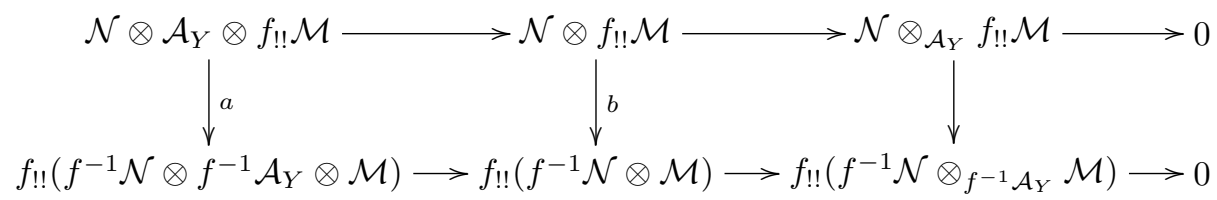

The top row of this diagram is exact by definition of the tensor product, as also is the bottom row, before we take the image under $f_{! !}$. But any complex of the type $\mathcal{P} \otimes \mathcal{M}$ is an $\mathcal{A}_{X}^{0}$-module because $\mathcal{M}$ is; hence it is $f_{\text {!!-acyclic by Proposition } 5.9}$ and Corollary 4.5. It follows that the bottom row is exact. Now, the vertical arrows $a$ and $b$ are isomorphisms in view of the classical projection formula. Hence so is the morphism of the lemma.

Base change. We consider a Cartesian square of real analytic manifolds

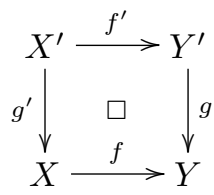

We have the usual base change formula in $\operatorname{Mod}\left(\mathbf{C}_{Y_{s a}^{\prime}}\right)$ or $\mathrm{C}^{+}\left(\mathbf{C}_{Y_{s a}^{\prime}}\right), f^{-1} g_{! !} \simeq$ $g_{! !}^{\prime} f^{\prime-1}$ (and its derived version in $\mathrm{D}^{+}\left(\mathbf{C}_{Y_{s a}^{\prime}}\right), f^{-1} \mathrm{R} g_{! !} \simeq \mathrm{R} g_{! !}^{\prime} f^{\prime-1}$ ).

Lemma 6.7. Let $\mathcal{N}$ be a $d g-\mathcal{A}_{Y^{\prime}}$-module. There exists a natural morphism

$$
f^{*} g_{! !} \mathcal{N} \rightarrow g_{! !}^{\prime} f^{\prime *} \mathcal{N}
$$

of $d g$ - $\mathcal{A}_{X}$-modules, whose image in $\mathrm{C}^{+}\left(\mathbf{C}_{X_{s a}}\right)$ gives a commutative diagram

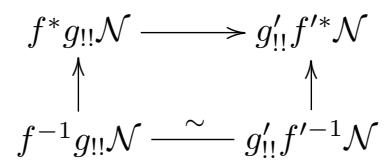

where the bottom arrow is the usual base change isomorphism. If $f$ is an immersion and $g$ is smooth, then (21) is an isomorphism.

Proof. The morphism is defined by the following composition:

$$
\begin{aligned}
f^{*} g_{! !} \mathcal{N} \simeq \mathcal{A}_{X} \otimes_{f^{-1}} \mathcal{A}_{Y} g_{! !}^{\prime} f^{\prime-1} \mathcal{N} \simeq g_{! !}^{\prime}\left(g^{\prime-1} \mathcal{A}_{X} \otimes_{g^{\prime-1} f^{-1} \mathcal{A}_{Y}} f^{\prime-1} \mathcal{N}\right) \\
\stackrel{\varphi}{\longrightarrow} g_{! !}^{\prime}\left(\mathcal{A}_{X^{\prime}} \otimes_{f^{\prime-1}} \mathcal{A}_{Y^{\prime}} f^{\prime-1} \mathcal{N}\right)=g_{! !}^{\prime} f^{\prime *} \mathcal{N}
\end{aligned}
$$


where the first isomorphism uses the classical base change formula (for complexes), and the second one the classical projection formula. The morphism $\varphi$ is induced by $g^{\prime \sharp}$

Now we show that $\varphi$ is an isomorphism when $f$ is an immersion and $g$ is smooth. It is enough to show that

$$
g^{\prime-1} \mathcal{A}_{X} \otimes_{g^{\prime-1} f^{-1}} \mathcal{A}_{Y} f^{\prime-1} \mathcal{N} \simeq \mathcal{A}_{X^{\prime}} \otimes_{f^{\prime-1}} \mathcal{A}_{Y^{\prime}} f^{\prime-1} \mathcal{N}
$$

This is a local statement on $X^{\prime}$ so that we may as well assume that $f$ is an embedding and $X^{\prime}=X \times Z, Y^{\prime}=Y \times Z$ for some manifold $Z$. We may also assume that $X$ is given by the equations $y_{i}=0, i=1, \ldots, d$, in $Y$. Then $\mathcal{A}_{X}$ is the quotient of $f^{-1} \mathcal{A}_{Y}$ by the ideal generated by $y_{i}, d y_{i}, i=1 \ldots, d$. The same holds for $X^{\prime}$ and we have the presentations

$$
\begin{aligned}
f^{-1}\left(\mathcal{A}_{Y}\right)^{2 d} \stackrel{\left(y_{1}, \ldots, d y_{d}\right)}{\longrightarrow} f^{-1}\left(\mathcal{A}_{Y}\right) \rightarrow \mathcal{A}_{X} \rightarrow 0, \\
f^{\prime-1}\left(\mathcal{A}_{Y^{\prime}}\right)^{2 d} \stackrel{\left(y_{1}, \ldots, d y_{d}\right)}{\longrightarrow} f^{\prime-1}\left(\mathcal{A}_{Y^{\prime}}\right) \rightarrow \mathcal{A}_{X^{\prime}} \rightarrow 0 .
\end{aligned}
$$

Since the tensor product is right exact, the images of these exact sequences under $g^{\prime-1}(\cdot) \otimes_{g^{\prime-1} f^{-1} \mathcal{A}_{Y}} f^{\prime-1} \mathcal{N}$ and $(\cdot) \otimes_{f^{\prime-1}} \mathcal{A}_{Y^{\prime}} f^{\prime-1} \mathcal{N}$ give the same presentations of both sides of 22 , which shows that they are isomorphic.

Complex manifolds. Now we assume that $X$ is a complex analytic manifold, of dimension $d_{X}^{c}$ over $\mathbf{C}$; we denote by $X_{\mathbf{R}}$ the underlying real analytic manifold. We recall that $t$ is the coordinate on $\widehat{X}_{\mathbf{R}}$ given by the projection $\widehat{X}_{\mathbf{R}} \rightarrow \mathbf{R}$, and that we have the decomposition $d=d_{1}+d_{2}$ of the differential of $\mathcal{A}_{X_{\mathbf{R}}}\left(d_{2}(\omega)=\partial \omega / \partial t d t\right)$. We consider the complex of "tempered holomorphic functions", $\mathcal{O}_{X}^{t} \in \mathrm{D}^{b}\left(\mathbf{C}_{X_{s a}}\right)$, defined as the Dolbeault complex with tempered coefficients

$$
\mathcal{O}_{X}^{t}=0 \rightarrow \Omega_{X_{\mathbf{R}}}^{t, 0,0} \stackrel{\bar{\partial}}{\rightarrow} \Omega_{X_{\mathbf{R}}}^{t, 0,1} \stackrel{\bar{\partial}}{\rightarrow} \cdots \stackrel{\bar{\partial}}{\rightarrow} \Omega_{X_{\mathbf{R}}}^{t, 0, d_{X}^{c}},
$$

where $\Omega_{X}^{t, i, j}$ denotes as usual the forms of type $(i, j)$. The product of forms induces a morphism $\mathcal{O}_{X}^{t} \otimes \mathcal{O}_{X}^{t} \rightarrow \mathcal{O}_{X}^{t}$ in $\mathrm{D}^{b}\left(\mathbf{C}_{X_{s a}}\right)$. In degree $0, H^{0}\left(\mathcal{O}_{X}^{t}\right)$ is a subalgebra of $\rho_{*} \mathcal{O}_{X}$.

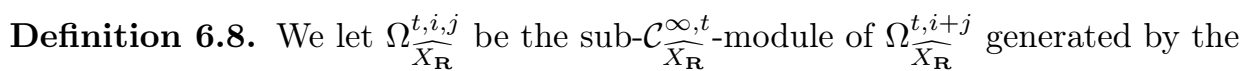
forms of type $(i, j)$ coming from $X_{\mathbf{R}}$.

We define $\mathcal{A}_{X_{\mathbf{R}}}^{i, j}=i_{X_{\mathbf{R}}}^{-1} \Gamma_{X_{\mathbf{R}}^{+}} \Omega_{X_{\mathbf{R}}}^{t, i, j}$. This is a sub- $\mathcal{A}_{X_{\mathbf{R}}}^{0}$-module of $\mathcal{A}_{X_{\mathbf{R}}}^{i+j}$ and we have the decomposition $\mathcal{A}_{X_{\mathbf{R}}}^{k}=\bigoplus_{i+j=k} \mathcal{A}_{X_{\mathbf{R}}}^{i, j} \oplus \bigoplus_{i+j=k-1} \mathcal{A}_{X_{\mathbf{R}}}^{i, j} d t$. The operators $\partial, \bar{\partial}$ on $\Omega_{X_{\mathbf{R}}}^{t}$ induce a decomposition of the differential of $\mathcal{A}_{X_{\mathbf{R}}}, d=\partial+\bar{\partial}+d_{2}$.

We let $J_{X} \subset \mathcal{A}_{X_{\mathbf{R}}}$ be the differential ideal generated by $\mathcal{A}_{X_{\mathrm{R}}}^{1,0}$ and introduce the $\operatorname{dg}-\mathcal{A}_{X_{\mathbf{R}}}$-module $\mathbb{O}_{X}=\mathcal{A}_{X_{\mathbf{R}}} / J_{X}$. As a quotient by a differential ideal, $\mathbb{O}_{X}$ inherits 
a structure of dg-algebra. We note the obvious inclusions $\rho_{!} \mathcal{O}_{X} \subset \rho_{!} \mathcal{C}_{X}^{\infty, t} \subset \mathcal{A}_{X_{\mathbf{R}}}^{0}$ and we define, for two complex analytic manifolds, $X, Y$,

$$
\mathbb{O}_{X}^{(i)}=\mathbb{O}_{X} \otimes_{\rho !} \mathcal{O}_{X} \rho_{!} \mathcal{O}_{X}^{(i)}, \quad \mathbb{O}_{X \times Y}^{(p, q)}=\mathbb{O}_{X \times Y} \otimes_{\rho_{!}\left(\mathcal{O}_{X} \otimes \mathcal{O}_{Y}\right)} \rho_{!}\left(\mathcal{O}_{X}^{(p)} \otimes \mathcal{O}_{Y}^{(q)}\right),
$$

where $\mathcal{O}_{X}^{(i)}$ denotes the holomorphic $i$-forms on $X$.

Proposition 6.9. (i) We have an isomorphism of complexes between $\mathbb{O}_{X}$ and

$$
\mathcal{A}_{X_{\mathbf{R}}}^{0,0} \rightarrow\left(\mathcal{A}_{X_{\mathbf{R}}}^{0,1} \oplus \mathcal{A}_{X_{\mathbf{R}}}^{0,0} d t\right) \rightarrow\left(\mathcal{A}_{X_{\mathbf{R}}}^{0,2} \oplus \mathcal{A}_{X_{\mathbf{R}}}^{0,1} d t\right) \rightarrow \cdots \rightarrow \mathcal{A}_{X_{\mathbf{R}}}^{0, d_{X}^{c}} d t
$$

with differential $\bar{\partial}+d_{2}$.

(ii) $\mathbb{O}_{X}^{\left(d_{X}^{c}\right)}\left[-d_{X}^{c}\right]$ is isomorphic to the differential ideal of $\mathcal{A}_{X_{\mathbf{R}}}$,

$$
\mathcal{A}_{X_{\mathbf{R}}}^{d_{X}^{c}, 0} \rightarrow\left(\mathcal{A}_{X_{\mathbf{R}}}^{d_{X}^{c}, 1} \oplus \mathcal{A}_{X_{\mathbf{R}}}^{d_{X}^{c}, 0} d t\right) \rightarrow \cdots \rightarrow \mathcal{A}_{X_{\mathbf{R}}}^{d_{X}^{c}, d_{X}^{c}} d t
$$

Moreover, setting $M_{X}=\bigoplus_{i<d_{X}^{c}, j \leq d_{X}^{c}}\left(\mathcal{A}_{X_{\mathbf{R}}}^{i, j} \oplus \mathcal{A}_{X_{\mathbf{R}}}^{i, j} d t\right)$, we have a decomposition $\mathcal{A}_{X_{\mathbf{R}}} \simeq \mathbb{O}_{X}^{\left(d_{X}^{c}\right)}\left[-d_{X}^{c}\right] \oplus M_{X}$ into locally free $\mathcal{A}_{X_{\mathbf{R}}}^{0}$-modules.

(iii) There exists a natural isomorphism $\mathcal{O}_{X}^{t} \simeq \mathbb{O}_{X}$ in $\mathrm{D}^{b}\left(\mathbf{C}_{\left(X_{\mathbf{R}}\right)_{s a}}\right)$ which commutes with the products $\mathcal{O}_{X}^{t} \otimes \mathcal{O}_{X}^{t} \rightarrow \mathcal{O}_{X}^{t}$ and $\mathbb{O}_{X} \otimes \mathbb{O}_{X} \rightarrow \mathbb{O}_{X}$. We also have $\mathcal{O}_{X \times Y}^{t(p, q)} \simeq \mathbb{O}_{X \times Y}^{(p, q)}$ in $\mathrm{D}^{b}\left(\mathbf{C}_{\left(X_{\mathbf{R}} \times Y_{\mathbf{R}}\right)_{s a}}\right)$.

Proof. The decomposition of $\mathcal{A}_{X_{\mathbf{R}}}^{k}$ given in Definition 6.8 yields projections $\mathcal{A}_{X_{\mathbf{R}}}^{k}$ $\rightarrow \mathcal{A}_{X_{\mathbf{R}}}^{0, k} \oplus \mathcal{A}_{X_{\mathbf{R}}}^{0, k-1} d t$. The sum of these projections is a surjective morphism from $\mathcal{A}_{X_{\mathbf{R}}}$ to the complex in (i) and we see that its kernel is $J_{X}$. This gives (i), and (ii) follows. We obtain (iii) by the exact sequences

$$
0 \rightarrow \Omega_{X_{\mathbf{R}}}^{t, 0, j} \rightarrow \mathcal{A}_{X_{\mathbf{R}}}^{0, j} \stackrel{\alpha \mapsto(\partial \alpha / \partial t) d t}{\longrightarrow} \mathcal{A}_{X_{\mathbf{R}}}^{0, j} d t \rightarrow 0
$$

and the definition of $\mathcal{O}_{X}^{t}$.

For a morphism of complex analytic manifolds $f: X \rightarrow Y$, we have a "tempered" integration morphism in the derived category, $\mathrm{R} f_{! !} \mathcal{O}_{X}^{t\left(d_{X}^{c}\right)}\left[d_{X}^{c}\right] \rightarrow$ $\mathcal{O}_{Y}^{t\left(d_{Y}^{c}\right)}\left[d_{Y}^{c}\right]$. When $f$ is a submersion, using the adjunction between $\mathrm{R} f_{! !}$and $f^{!} \simeq f^{-1}\left[2\left(d_{X}^{c}-d_{Y}^{c}\right)\right]$, it can be written

$$
\mathcal{O}_{X}^{t\left(d_{X}^{c}\right)}\left[-d_{X}^{c}\right] \rightarrow f^{-1} \mathcal{O}_{Y}^{t\left(d_{Y}^{c}\right)}\left[-d_{Y}^{c}\right]
$$

Proposition 6.10. Let $f: X \rightarrow Y$ be a submersion. The embeddings of Proposition 6.9 (ii), $\mathbb{O}_{Z}^{\left(d_{Z}^{c}\right)}\left[-d_{Z}^{c}\right] \subset \mathcal{A}_{Z_{\mathbf{R}}}($ for $Z=X, Y)$, induce a morphism of $d g-\mathcal{A}_{X_{\mathbf{R}}}{ }^{-}$ modules

$$
\mathbb{O}_{X}^{\left(d_{X}^{c}\right)}\left[-d_{X}^{c}\right] \rightarrow f^{*} \mathbb{O}_{Y}^{\left(d_{Y}^{c}\right)}\left[-d_{Y}^{c}\right]
$$

which represents (24) through the isomorphism of Proposition 6.9(iii). 
Proof. By Proposition 6.9 we have a decomposition $\mathcal{A}_{Y_{\mathbf{R}}} \simeq \mathbb{O}_{Y}^{\left(d_{Y}^{c}\right)}\left[-d_{Y}^{c}\right] \oplus M_{Y}$ into locally free $\mathcal{A}_{Y_{\mathbf{R}}}^{0}$-modules; hence the quotient $\mathcal{A}_{Y_{\mathbf{R}}} / \mathbb{O}_{Y}^{\left(d_{Y}^{c}\right)}\left[-d_{Y}^{c}\right]$ is flat over $\mathcal{A}_{Y_{\mathbf{R}}}^{0}$ and Proposition 6.3 implies that the morphism $f^{*} \mathbb{O}_{Y}^{\left(d_{Y}^{c}\right)}\left[-d_{Y}^{c}\right] \rightarrow f^{*} \mathcal{A}_{Y_{\mathbf{R}}} \simeq$ $\mathcal{A}_{X_{\mathbf{R}}}$ is injective. Hence we just have to check the inclusion of ideals of $\mathcal{A}_{X_{\mathbf{R}}}$ : $\mathbb{O}_{X}^{\left(d_{X}^{c}\right)}\left[-d_{X}^{c}\right] \subset f^{*} \mathbb{O}_{Y}^{\left(d_{Y}^{c}\right)}\left[-d_{Y}^{c}\right]$. This is a local problem on $X$ which can be checked in a local coordinate system.

With the hypothesis of Proposition 6.10, we could have defined an integration morphism $f_{! !} \mathbb{O}_{X}^{\left(d_{X}^{c}\right)}\left[d_{X}^{c}\right] \rightarrow \mathbb{O}_{Y}^{\left(d_{Y}^{c}\right)}\left[d_{Y}^{c}\right]$.

In Section 10 we need the following composition of kernels. Let $X, Y, Z$ be three complex analytic manifolds and $q_{i j}$ the projection from their product to the $i^{\text {th }} \times j^{\text {th }}$ factors. The product of $\mathcal{O}_{Y}^{t}$ and the integration morphism give a convolution product

$$
\operatorname{R} q_{13 !}\left(q_{12}^{-1} \mathcal{O}_{X \times Y}^{\left(0, d_{Y}^{c}\right)}\left[d_{Y}^{c}\right] \otimes q_{23}^{-1} \mathcal{O}_{Y \times Z}^{\left(0, d_{Z}^{c}\right)}\left[d_{Z}^{c}\right]\right) \rightarrow \mathcal{O}_{X \times Z}^{\left(0, d_{Z}^{c}\right)}\left[d_{Z}^{c}\right]
$$

We can realize this tempered convolution product on the resolutions $\mathbb{O}_{X \times Y}^{\left(0, d_{Y}^{c}\right)}$, but in fact we will rather need its "adjoint" morphism

$$
\begin{aligned}
q_{12}^{*} \mathbb{O}_{X \times Y}^{\left(0, d_{Y}^{c}\right)}\left[-d_{Y}^{c}\right] \otimes_{\mathcal{A}} q_{23}^{*} \mathbb{O}_{Y \times Z}^{\left(0, d_{Z}^{c}\right)}\left[-d_{Z}^{c}\right] \rightarrow \mathbb{O}_{X \times Y \times Z}^{\left(0, d_{Y}^{c}, d_{Z}^{c}\right)}\left[-d_{Y}^{c}-d_{Z}^{c}\right] & \rightarrow q_{13}^{*} \mathbb{O}_{X \times Z}^{\left(0, d_{Z}^{c}\right)}\left[-d_{Z}^{c}\right]
\end{aligned}
$$

where the first morphism is induced by the product $\mathbb{O}_{Y} \otimes \mathbb{O}_{Y} \otimes_{\rho_{!} \mathcal{O}_{Y}} \rho_{!} \mathcal{O}_{Y}^{\left(d_{Y}^{c}\right)} \rightarrow$ $\mathbb{O}_{Y} \otimes_{\rho_{!} \mathcal{O}_{Y}} \rho_{!} \mathcal{O}_{Y}^{\left(d_{Y}^{c}\right)}$ and the second morphism is induced by 25 .

\section{§7. Microlocalization functor}

In this section we recall the definition of the microlocalization functor $\mu$ introduced in [9]. For a manifold $X$ this is a functor, $\mu_{X}$, from $\mathrm{D}^{b}\left(\mathbf{I}\left(\mathbf{C}_{X}\right)\right)$ to $\mathrm{D}^{b}\left(\mathbf{I}\left(\mathbf{C}_{T^{*} X}\right)\right)$ given by a kernel $L_{X} \in \mathrm{D}^{b}\left(\mathbf{I}\left(\mathbf{C}_{X \times T^{*} X}\right)\right)$.

We define an analog of this kernel and of the microlocalization functor in the framework of $\mathcal{A}$-modules. We check that in the case we are interested in, this gives a quasi-injective resolution of $\mu_{X} F$, and that it has a functorial behavior with respect to the usual operations.

In fact, with the definition of [9] the construction of the external tensor product is not so straightforward. For this reason we define another kernel for which the tensor product is easy and which coincides with the kernel of [9] outside the zero section. 


\section{§7.1. Microlocalization functor in the derived category}

In [9] the authors define a kernel associated to the following data: let $X$ be a manifold, $Z \subset X$ a closed submanifold and $\sigma$ a 1-form defined on $Z$, i.e. $\sigma$ is a section of the bundle $Z \times_{X} T^{*} X \rightarrow Z$. To simplify the exposition we make the following assumption which will be satisfied in our case:

$$
\text { for any } z \in Z, \sigma_{z} \text { is non-zero but } \sigma_{z}(\nu)=0, \forall \nu \in T_{z} Z \text {. }
$$

Hence $\sigma$ induces a non-vanishing section of $T_{Z}^{*} X \rightarrow Z$ and we may define

$$
P_{\sigma}^{0}=\left\{(x, v) \in T_{Z} X ;\langle v, \sigma(x)\rangle>0\right\}, \quad P_{\sigma}=\overline{P_{\sigma}^{0}} .
$$

Thus $P_{\sigma}$ is a subset of $T_{Z} X$, viewed itself as a subset of the normal deformation of $Z$ in $X, \tilde{X}_{Z}$. We recall that $\tilde{X}_{Z}$ and the projection $p: \tilde{X}_{Z} \rightarrow X$ are given in local coordinates as follows. We choose coordinates $\left(x_{1}, \ldots, x_{n}\right)$ on $X$ such that $Z$ is given by $x_{i}=0, i=1, \ldots, d$. This gives coordinates $\left(x_{i}, \tau\right)$ on $\tilde{X}_{Z}$ and $p\left(x_{i}, \tau\right)=\left(\tau x_{1}, \ldots, \tau x_{d}, x_{d+1}, \ldots, x_{n}\right)$. The normal bundle $T_{Z} X$ is embedded in $\tilde{X}_{Z}$ as the submanifold $\{\tau=0\}$ and we define $\Omega=\{\tau>0\}$ :

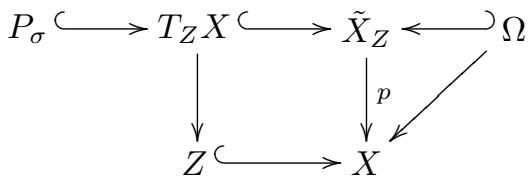

In 9] the objects are introduced in the category of ind-sheaves but we will work on the subanalytic site, using the embedding of categories $\mathbf{I}_{\tau}: \operatorname{Mod}\left(\mathbf{C}_{X_{s a}}\right) \simeq$ $\mathbf{I}_{\mathbf{R}-c}\left(\mathbf{C}_{X}\right) \rightarrow \mathbf{I}\left(\mathbf{C}_{X}\right)$. Moreover, under hypothesis 28 we may use the following definition for the kernel instead of Definition 1.2.3 of [9] (see Proposition 1.2.11, loc. cit.).

Definition 7.1. The kernel associated to the above data is the complex $\mathcal{L}_{\sigma}=$ $\mathbf{I}_{\tau}\left(\mathcal{L}_{\sigma}^{s a}\right) \in \mathrm{D}^{b}\left(\mathbf{I}\left(\mathbf{C}_{X}\right)\right)$, where $\mathcal{L}_{\sigma}^{s a} \in \mathrm{D}^{b}\left(\mathbf{C}_{X_{s a}}\right)$ is given by (recall that, for $i: Z \hookrightarrow$ $X$, we write $\omega_{Z \mid X}$ instead of $\left.i_{*} \omega_{Z \mid X}\right)$

$$
\mathcal{L}_{\sigma}^{s a}=\mathrm{R} p_{! !}\left(\rho_{\tilde{X}_{Z} !}\left(\mathbf{C}_{P_{\sigma}}\right) \otimes \mathbf{C}_{\Omega}\right) \otimes \rho_{X !}\left(\omega_{Z \mid X}^{\otimes-1}\right)
$$

We note that $\mathrm{R} p_{! !}\left(\rho_{!}\left(\mathbf{C}_{P_{\sigma}}\right) \otimes \mathbf{C}_{\Omega}\right)$ is supported on $Z$ (i.e. its restriction outside $Z$ is 0$)$. Hence taking the tensor product with $\rho_{!}\left(\omega_{Z \mid X}^{\otimes-1}\right)$ reduces locally to a shift by the codimension of $Z$. By Proposition 1.2.11 of [9] we also have

$$
\mathrm{R} p_{! !}\left(\rho_{\tilde{X}_{Z} !}\left(\mathbf{C}_{P_{\sigma}}\right) \otimes \mathbf{C}_{\Omega}\right) \simeq \underset{U}{ } \underset{ }{\text { lim" }} \mathbf{C}_{U} \otimes \rho_{X !}\left(\mathbf{C}_{Z}\right),
$$


where $U$ runs over the open subsets of $X$ such that the cone of $U$ along $Z$ does not intersect $P_{\sigma}$ outside the zero section. In particular the complexes in 29 are concentrated in degree 0: $\operatorname{R} p_{! !}\left(\rho_{\tilde{X}_{Z} !}\left(\mathbf{C}_{P_{\sigma}}\right) \otimes \mathbf{C}_{\Omega}\right) \simeq p_{! !}\left(\rho_{\tilde{X}_{Z} !}\left(\mathbf{C}_{P_{\sigma}}\right) \otimes \mathbf{C}_{\Omega}\right)$.

When considering resolutions of $\mathcal{L}_{\sigma}$ by $\mathcal{A}$-modules it will be convenient to use the following different formulation.

Definition 7.2. For an analytic manifold $Y$ and $T \subset Y$ a locally closed subanalytic subset we define $K_{T} \in \operatorname{Mod}\left(\mathbf{C}_{Y_{s a}}\right)$ by $K_{T}=\varliminf_{W W, W^{0}} \mathbf{C}_{\bar{W} \backslash \bar{W}^{0}}$, where $W$ (resp. $W^{0}$ ) runs over the open neighborhoods of $\bar{T}$ (resp. $T$ ) in $Y$. We note that $K_{T}$ has support in the boundary $\partial T=\bar{T} \backslash T$.

Lemma 7.3. Let $(X, Z, \sigma)$ be a kernel data satisfying (28). We have an isomorphism in $\mathrm{D}^{b}\left(\mathbf{C}_{X_{s a}}\right)$,

$$
\mathcal{L}_{\sigma}^{s a} \simeq \mathrm{R} p_{! !}\left(K_{P_{\sigma}^{0}} \otimes \mathbf{C}_{\Omega}\right) \otimes \rho_{X !}\left(\omega_{Z \mid X}^{\otimes-1}\right) .
$$

Proof. We define $F=\lim _{W^{0}} \mathbf{C}_{\overline{W^{0}}}$, where $W^{0}$ runs over the open neighborhoods of $P_{\sigma}^{0}$ in $\tilde{X}_{Z}$. Hence we have an exact sequence $0 \rightarrow K_{P_{\sigma}^{0}} \rightarrow \rho_{\tilde{X}_{Z} !}\left(\mathbf{C}_{P_{\sigma}}\right) \rightarrow F \rightarrow 0$ and it is enough to show that $\mathrm{R} p_{! !}\left(F \otimes \mathbf{C}_{\Omega}\right)=0$.

We have $\mathrm{R} p_{! !}\left(F \otimes \mathbf{C}_{\Omega}\right) \simeq{\underline{\lim _{W}, U}} \mathrm{R} p_{*} \mathbf{C}_{\overline{W^{0}} \cap \Omega \cap U}$, where $W^{0}$ runs over the same set as above and $U$ runs over the open subsets of $\tilde{X}_{Z}$ with compact closure. Since $p_{*}$ commutes with $\rho_{*}$ we are reduced to a computation with sheaves on topological spaces.

For $x$ in $X \backslash Z$, near $Z, p^{-1}(x) \cap \overline{W^{0}} \cap \Omega \cap U$ is a union of intervals of the line, all compact except at most one which is homeomorphic to $[0,1[$. When we take the limit over $W^{0}$ and $U$ only the last one has a non-zero contribution to the morphisms $\mathbf{C}_{p^{-1}(x) \cap \overline{W^{0}} \cap \Omega \cap U} \rightarrow \mathbf{C}_{p^{-1}(x) \cap \overline{W^{\prime 0}} \cap \Omega \cap U^{\prime}}$. Since $\operatorname{R} \Gamma\left(\mathbf{R} ; \mathbf{C}_{[0,1[}\right)=0$ we deduce that our direct image vanishes.

Now, for any manifold $X$ the cotangent bundle $T^{*} X$ is endowed with a canonical 1-form, say $\omega_{X}$. We restrict outside the zero-section and set $\mathfrak{X}=X \times \dot{T}^{*} X, \mathfrak{Z}=$ $X \times_{X} \dot{T}^{*} X \simeq \dot{T}^{*} X$ and consider the section $\sigma_{X}: X \times_{X} \dot{T}^{*} X \rightarrow T^{*} X \times T^{*}\left(\dot{T}^{*} X\right)$ defined by $\sigma_{X}=\left(-\mathrm{id}, \omega_{X}\right)$, i.e. in local coordinates

$$
\sigma_{X}(x, x, \xi)=\left((x ;-\xi), \omega_{X}(x, \xi)\right)=((x ;-\xi),(x, \xi ; \xi, 0)) .
$$

Hence hypothesis $(28)$ is satisfied for the data $\left(\mathfrak{X}, \mathfrak{Z}, \sigma_{X}\right)$.

Definition 7.4. With the above notations, we set $L_{X}=\mathcal{L}_{\sigma_{X}}$ so that $L_{X} \in$ $\mathrm{D}^{b}\left(\mathbf{I}\left(\mathbf{C}_{X \times \dot{T}^{*} X}\right)\right)$. We denote by $p_{1}: X \times \dot{T}^{*} X \rightarrow X, p_{2}: X \times \dot{T}^{*} X \rightarrow \dot{T}^{*} X$ the projections. The microlocalization is the functor

$$
\mu_{X}: \mathrm{D}^{b}\left(\mathbf{I}\left(\mathbf{C}_{X}\right)\right) \rightarrow \mathrm{D}^{b}\left(\mathbf{I}\left(\mathbf{C}_{\dot{T}^{*} X}\right)\right), \quad F \mapsto L_{X} \circ F=\mathrm{R} p_{2 ! !}\left(L_{X} \otimes p_{1}^{-1} F\right) .
$$




\section{§7.2. Microlocalization functor for $\mathcal{A}$-modules}

Definition 7.5. With the notations of Definition 7.2, we define $\mathcal{B}_{T} \in \operatorname{Mod}\left(\mathcal{A}_{Y}\right)$ by $\mathcal{B}_{T}=\lim _{W, W^{0}} \Gamma_{Y \backslash W^{0}}\left(\mathcal{A}_{Y} \otimes \mathbf{C}_{\bar{W}}\right)$, where $W$ (resp. $W^{0}$ ) runs over the open neighborhoods of $\bar{T}$ (resp. $T$ ) in $Y$.

For an open subset $U \subset Y$, we denote by $\mathbf{C}_{U}^{\prime}$ the complex $\mathbf{C}_{\bar{U}} \rightarrow \mathbf{C}_{\partial U}$ in $\mathrm{C}^{+}\left(\mathbf{C}_{Y_{s a}}\right)$, with $\mathbf{C}_{\bar{U}}$ in degree 0 . We have a quasi-isomorphism $\mathbf{C}_{U} \rightarrow \mathbf{C}_{U}^{\prime}$.

For a kernel data $(X, Z, \sigma)$ we define $\mathcal{L}_{\sigma}^{\mathcal{A}} \in \operatorname{Mod}\left(\mathcal{A}_{X}\right)$ by

$$
\mathcal{L}_{\sigma}^{\mathcal{A}}=p_{! !}\left(\mathcal{B}_{P_{\sigma}^{0}} \otimes \mathbf{C}_{\Omega}^{\prime}\right) \otimes \rho_{X !}\left(\omega_{Z \mid X}^{\otimes-1}\right) .
$$

Remark 7.6. For $V \subset Y$ open, a section of $\mathcal{B}_{T}(V)$ is represented by an element $a \in \Gamma\left(V \cap W ; \mathcal{A}_{Y}\right)$ such that $\left.a\right|_{W^{0}}=0$, where $W$ and $W^{0}$ are some neighborhoods of $\bar{T}$ and $T$.

Lemma 7.7. (i) Let $Y$ be a real analytic manifold and $T \subset Y$ be a locally closed subset whose embedding in $Y$ is locally homeomorphic to the embedding of a convex set in $\mathbf{R}^{n}$. Then in $\mathrm{D}^{b}\left(\mathbf{C}_{Y_{s a}}\right)$ we have an isomorphism $K_{T} \stackrel{\sim}{\rightarrow} \mathcal{B}_{T}$ and $\mathcal{B}_{T}$ consists of quasi-injective sheaves on $Y_{\text {sa }}$.

(ii) For a kernel data $(X, Z, \sigma)$ satisfying hypothesis (28), (i) induces an isomorphism $\mathcal{L}_{\sigma} \simeq \mathcal{L}_{\sigma}^{\mathcal{A}}$ in $\mathrm{D}^{+}\left(\mathbf{I}\left(\mathbf{C}_{X}\right)\right)$.

(iii) $\mathcal{L}_{\sigma}^{\mathcal{A}}$ is a complex of quasi-injective sheaves on $X_{\text {sa }}$.

(iv) For $F \in \operatorname{Mod}\left(\mathcal{A}_{X}\right)$, the morphism $\mathcal{L}_{\sigma}^{\mathcal{A}} \otimes F \rightarrow \mathcal{L}_{\sigma}^{\mathcal{A}} \otimes_{\mathcal{A}_{X}} F$ in $\mathrm{C}^{+}\left(\mathbf{C}_{X_{\text {sa }}}\right)$ is a quasi-isomorphism.

Proof. (i) In Definition 7.2 we may as well assume that the embedding of $W^{0}$ in $Y$ is locally homeomorphic to the embedding of an open convex set in $\mathbf{R}^{n}$, so that $\mathbf{C}_{Y \backslash \overline{W^{0}}} \simeq \mathrm{R} \Gamma_{Y \backslash W^{0}} \mathbf{C}_{Y}$. Since $\mathcal{A}_{Y}$ is a quasi-injective resolution of $\mathbf{C}_{Y}$ we deduce $\mathbf{C}_{Y \backslash \overline{W^{0}}} \simeq \Gamma_{Y \backslash W^{0}} \mathcal{A}_{Y}$. Now we take the tensor product with $\rho_{!} \mathbf{C}_{\bar{T}}$ and use formula (6) to obtain the isomorphism.

The quasi-injectivity of $\mathcal{B}_{T}$ follows from that of $\mathcal{A}_{Y}$ and the description of sections in Remark 7.6

(ii) By (i) we have $K_{P_{\sigma}^{0}} \otimes \mathbf{C}_{\Omega} \stackrel{\sim}{\rightarrow} \mathcal{B}_{P_{\sigma}^{0}} \otimes \mathbf{C}_{\Omega}^{\prime}$. By Proposition 5.9 and Corol-

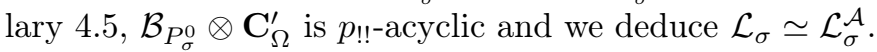

(iii) Since $\bar{\Omega}$ and $\partial \Omega$ are closed, (i) and Proposition 1.5.13 of [10] show that

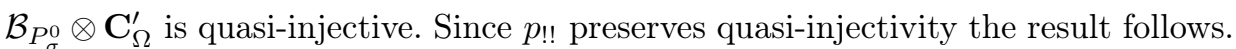

(iv) Since $\left.p\right|_{\Omega}: \Omega \rightarrow X$ is smooth Proposition 6.3 and Corollary 5.10 give an isomorphism $\left(\mathcal{B}_{P_{\sigma}^{0}} \otimes \mathbf{C}_{\Omega}\right) \otimes p^{-1} F \simeq\left(\mathcal{B}_{P_{\sigma}^{0}} \otimes \mathbf{C}_{\Omega}\right) \otimes_{p^{-1} \mathcal{A}_{X}} p^{-1} F$ in $\mathrm{D}^{+}\left(\mathbf{C}_{\left(\tilde{X}_{Z}\right)_{s a}}\right)$. We also have $\mathbf{C}_{\Omega} \stackrel{\sim}{\rightarrow} \mathbf{C}_{\Omega}^{\prime}$, so we may replace $\mathbf{C}_{\Omega}$ by $\mathbf{C}_{\Omega}^{\prime}$ in this isomorphism. We take the image under $p_{\text {!! }}$, and using the projection formula and the fact that

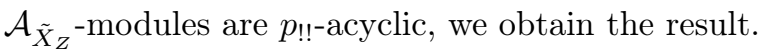


Now we can define the microlocalization functor for $\mathcal{A}$-modules. We keep the notations introduced before Definition 7.4 for a manifold $X$ we have the kernel $\operatorname{data}\left(\mathfrak{X}, \mathfrak{Z}, \sigma_{X}\right)$.

Definition 7.8. With the above notations, we set $L_{X}^{\mathcal{A}}=\mathcal{L}_{\sigma_{X}}^{\mathcal{A}}$ so that $L_{X}^{\mathcal{A}} \in$ $\operatorname{Mod}\left(\mathcal{A}_{X \times \dot{T}^{*} X}\right)$. The microlocalization is the functor

$$
\mu_{X}^{\mathcal{A}}: \operatorname{Mod}\left(\mathcal{A}_{X}\right) \rightarrow \operatorname{Mod}\left(\mathcal{A}_{\dot{T}^{*} X}\right), \quad F \mapsto L_{X}^{\mathcal{A}} \circ F=p_{2 ! !}\left(L_{X}^{\mathcal{A}} \otimes_{\mathcal{A}_{X}} p_{1}^{*} F\right)
$$

Proposition 7.9. (i) For $F \in \operatorname{Mod}\left(\mathcal{A}_{X}\right)$ there exists a natural isomorphism $\mu_{X}\left(\operatorname{For}_{X}^{\prime}(F)\right) \stackrel{\sim}{\rightarrow} \operatorname{For}_{T^{*} X}^{\prime}\left(\mu_{X}^{\mathcal{A}}(F)\right)$ in $\mathrm{D}^{+}\left(\mathbf{I}\left(\mathbf{C}_{T^{*} X}\right)\right)$.

(ii) If $F$ admits a presentation $\left(\mathcal{A}_{X}\right)^{N} \rightarrow F \rightarrow 0$ then $\mu_{X}^{\mathcal{A}}(F)$ is a complex of quasi-injective sheaves on $T^{*} X_{\text {sa }}$.

Proof. (i) By Lemma 7.7(ii) we have $\mu_{X} F \simeq \mathrm{R} p_{2 ! !}\left(L_{X}^{\mathcal{A}} \otimes p_{1}^{-1} F\right)$. Since $\mathcal{A}$-modules are soft we may replace $\mathrm{R} p_{2 ! !}$ by $p_{2 ! !}$. By Proposition 6.3 we also have $p_{1}^{-1} F \stackrel{\sim}{\rightarrow} p_{1}^{*} F$ and we conclude by Lemma 7.7(iv).

(ii) follows from Proposition 6.1 and the fact that $p_{2 ! !}$ preserves quasi-injectivity.

\section{§. Functorial behavior of the kernel}

We study the behavior of $\mathcal{L}_{\sigma}^{\mathcal{A}}$ under direct image, inverse image and external tensor product. The morphisms built in this section correspond to the morphisms of Propositions 1.3.1, 1.3.3, 1.3.4 and 1.3.8 of [9] through the isomorphism of Lemma 7.7(ii), but we have to make additional hypothesis on the maps.

In this section $\left(X_{1}, Z_{1}, \sigma_{1}\right)$ and $\left(X_{2}, Z_{2}, \sigma_{2}\right)$ are two sets of data as above, satisfying hypothesis 28 . We set for short $\tilde{X}_{i}={\left.\widetilde{\left(X_{i}\right.}\right)_{Z_{i}}}_{\text {. }}$.

Direct and inverse images. Let $f: X_{1} \rightarrow X_{2}$ be a morphism with $f\left(Z_{1}\right) \subset Z_{2}$ and $\sigma_{1}=f^{*} \sigma_{2}$. It induces $\tilde{f}: \tilde{X}_{1} \rightarrow \tilde{X}_{2}$, decomposed as $\tilde{f}=h \circ g$ in the following diagram where the square is Cartesian:

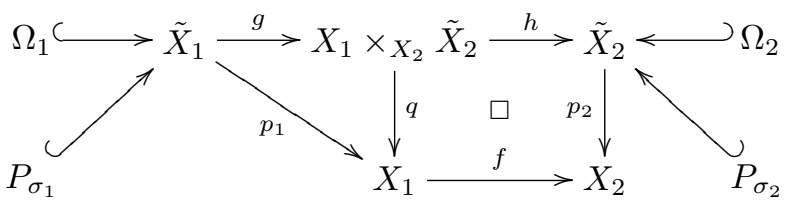

We have $\Omega_{1}=\tilde{f}^{-1} \Omega_{2}, T_{Z_{1}} X_{1}=\tilde{f}^{-1}\left(T_{Z_{2}} X_{2}\right), P_{\sigma_{1}}=\tilde{f}^{-1} P_{\sigma_{2}}, P_{\sigma_{1}}^{0}=\tilde{f}^{-1} P_{\sigma_{2}}^{0}$. We note that $X_{1} \times_{X_{2}} \tilde{X}_{2}$ is in general not a manifold and may have components of different dimensions. When $f$ is clean with respect to $Z_{2}$ and $Z_{1}=f^{-1}\left(Z_{2}\right)$ (clean 
then means that $g^{\prime}: T_{Z_{1}} X_{1} \rightarrow X_{1} \times_{X_{2}} T_{Z_{2}} X_{2}$ is injective), $g$ is a closed embedding. When $f$ is transversal to $Z_{2}$ and $Z_{1}=f^{-1}\left(Z_{2}\right), g$ is an isomorphism.

Proposition 8.1. Assume that $f$ and $\left.f\right|_{Z_{1}}: Z_{1} \rightarrow Z_{2}$ are smooth. Then there exists a natural morphism of $d g$ - $\mathcal{A}_{X_{2}}$-modules

$$
f_{! !}\left(\mathcal{L}_{\sigma_{1}}^{\mathcal{A}} \otimes \rho_{X_{1} !}\left(\omega_{Z_{1} \mid Z_{2}}\right)\right) \rightarrow \mathcal{L}_{\sigma_{2}}^{\mathcal{A}}
$$

Proof. The hypothesis implies that $\tilde{f}$ is smooth. Since $P_{\sigma_{1}}^{0}=\tilde{f}^{-1} P_{\sigma_{2}}^{0}$, the integration morphism of Definition 5.3 induces $\tilde{f}_{! !}\left(\mathcal{B}_{P_{\sigma_{1}}^{0}} \otimes \omega_{\tilde{X}_{1} \mid \tilde{X}_{2}}\right) \rightarrow \mathcal{B}_{P_{\sigma_{2}}^{0}}$ (this can be checked by using the description of sections of $\mathcal{B}_{T}$ in Remark 7.6). We also have $p_{1}^{-1} \omega_{X_{1} \mid X_{2}} \simeq \omega_{\tilde{X}_{1} \mid \tilde{X}_{2}}$ and $\tilde{f}^{-1} \mathbf{C}_{\Omega_{2}}^{\prime}=\mathbf{C}_{\Omega_{1}}^{\prime}$. This gives the morphisms

$$
\begin{aligned}
f_{! !}\left(p_{1 ! !}\left(\mathcal{B}_{P_{\sigma_{1}}^{0}} \otimes \mathbf{C}_{\Omega_{1}}^{\prime}\right) \otimes \omega_{X_{1} \mid X_{2}}\right) & \simeq p_{2 ! !} \tilde{f}_{! !}\left(\mathcal{B}_{P_{\sigma_{1}}^{0}} \otimes \tilde{f}^{-1} \mathbf{C}_{\Omega_{2}}^{\prime} \otimes \omega_{\tilde{X}_{1} \mid \tilde{X}_{2}}\right) \\
& \rightarrow p_{2 ! !}\left(\mathcal{B}_{P_{\sigma_{2}}^{0}} \otimes \mathbf{C}_{\Omega_{2}}^{\prime}\right)
\end{aligned}
$$

where in the first line we use the projection formula for $p_{1}$ and $f p_{1}=p_{2} \tilde{f}$. In the second line we use the projection formula for $\tilde{f}$ and the above morphism $\tilde{f}_{! !}\left(\mathcal{B}_{P_{\sigma_{1}}^{0}} \otimes \omega_{\tilde{X}_{1} \mid \tilde{X}_{2}}\right) \rightarrow \mathcal{B}_{P_{\sigma_{2}}^{0}}$.

Now we take the tensor product with $\omega_{Z_{2} \mid X_{2}}^{\otimes-1}$ and we obtain (31.

Proposition 8.2. Assume that $Z_{1}=f^{-1}\left(Z_{2}\right)$ and $f$ is clean with respect to $Z_{2}$. Then there exists a natural morphism of $d g$ - $\mathcal{A}_{X_{1}}$-modules

$$
f^{*} \mathcal{L}_{\sigma_{2}}^{\mathcal{A}} \rightarrow \mathcal{L}_{\sigma_{1}}^{\mathcal{A}} \otimes \rho_{X_{1} !}\left(\omega_{Z_{1} \mid Z_{2}}\right) \otimes \omega_{X_{1} \mid X_{2}}^{\otimes-1}
$$

If $f$ is transversal to $Z_{2}$ the morphism becomes $f^{*} \mathcal{L}_{\sigma_{2}}^{\mathcal{A}} \rightarrow \mathcal{L}_{\sigma_{1}}^{\mathcal{A}}$.

Proof. The hypotheses imply that the morphism $g$ in diagram $\sqrt{30}$ is an embedding. Hence $g_{*}=g_{! !}$and we have the adjunction morphism id $\rightarrow g_{! !} g^{-1}$. We compose it with the base change $f^{-1} p_{2 ! !} \rightarrow q_{! !} h^{-1}$ to obtain

$$
f^{-1} p_{2 ! !} \rightarrow q_{! !} g_{! !} g^{-1} h^{-1}=p_{1 ! !} \tilde{f}^{-1} .
$$

We apply $\mathcal{A}_{X_{1}} \otimes_{f^{-1}} \mathcal{A}_{X_{2}}(\cdot)$ to this morphism and use the projection formula for $p_{1}$ to obtain a morphism $f^{*} p_{2 ! !} \rightarrow p_{1 ! !} \tilde{f}^{*}$. It induces the first arrow in the sequence below. The second arrow is induced by $\tilde{f}^{-1} \mathbf{C}_{\Omega_{2}}^{\prime}=\mathbf{C}_{\Omega_{1}}^{\prime}$ and the inverse image $\tilde{f}^{\sharp}$. The composition defines (32):

$$
\begin{aligned}
f^{*} \mathcal{L}_{\sigma_{2}}^{\mathcal{A}} & =f^{*}\left(p_{2 ! !}\left(\mathcal{B}_{P_{\sigma_{2}}^{0}} \otimes \mathbf{C}_{\Omega_{2}}^{\prime}\right) \otimes \rho_{X_{2}} ! \omega_{Z_{2} \mid X_{2}}^{\otimes-1}\right) \\
& \rightarrow\left(p_{1 ! !} \tilde{f}^{*}\left(\mathcal{B}_{P_{\sigma_{2}}^{0}} \otimes \mathbf{C}_{\Omega_{2}}^{\prime}\right)\right) \otimes f^{-1} \rho_{X_{2} !} \omega_{Z_{2} \mid X_{2}}^{\otimes-1} \\
& \rightarrow p_{1 ! !}\left(\mathcal{B}_{P_{\sigma_{1}}^{0}} \otimes \mathbf{C}_{\Omega_{1}}^{\prime}\right) \otimes f^{-1} \rho_{X_{2} !} \omega_{Z_{2} \mid X_{2}}^{\otimes-1}
\end{aligned}
$$

If $f$ is transversal to $Z_{2}$ we have $\omega_{Z_{1} \mid X_{1}} \simeq f^{-1} \omega_{Z_{2} \mid X_{2}}$. 
External tensor product. The external tensor product is a consequence of Proposition 1.3 .8 of $[9]$. We give a slightly different proof here using the modification in our definition of $\mathcal{L}_{\sigma}$. In particular we use the following lemma.

Lemma 8.3. Let $Y_{i}, i=1,2$, be real analytic manifolds, and $T_{i} \subset Y_{i}$ and $T \subset$ $Y_{1} \times Y_{2}$ be locally closed subanalytic subsets such that

$$
T \subset\left(T_{1} \times Y_{2}\right) \cup\left(Y_{1} \times T_{2}\right) \quad \text { and } \quad \bar{T} \subset\left(T_{1} \times Y_{2}\right) \cup\left(Y_{1} \times T_{2}\right) \cup\left(\bar{T}_{1} \times \bar{T}_{2}\right) \text {. }
$$

Then there exist morphisms $K_{T_{1}} \otimes K_{T_{2}} \rightarrow K_{T}$ in $\mathrm{C}\left(\mathbf{C}_{\left(Y_{1} \times Y_{2}\right)_{s a}}\right)$ and $\mathcal{B}_{T_{1}} \otimes \mathcal{B}_{T_{2}} \rightarrow \mathcal{B}_{T}$ in $\operatorname{Mod}\left(\mathcal{A}_{Y_{1} \times Y_{2}}\right)$ which coincide in the derived category.

Proof. We only define the morphism for $\mathcal{B}$., the construction for $K$. being similar. We recall that a section of $\mathcal{B}_{T_{i}}$ over $U_{i} \subset Y_{i}$ is represented by $a_{i} \in \Gamma\left(U_{i} \cap W_{i} ; \mathcal{A}_{Y_{i}}\right)$ such that $\left.a_{i}\right|_{W_{i}^{0}}=0$, for some neighborhoods $W_{i}$ and $W_{i}^{0}$ of $\bar{T}_{i}$ and $T_{i}$ in $Y_{i}$. Hence $a=a_{1} \cdot a_{2}$ gives a section of $\mathcal{A}_{Y_{1} \times Y_{2}}$ over $W_{1} \times W_{2}$ such that $\left.a\right|_{\left(W_{1} \times W_{2}\right) \cap W^{0}}=0$, where $W^{0}=\left(W_{1}^{0} \times Y_{2}\right) \cup\left(Y_{1} \times W_{2}^{0}\right)$. Hence we may extend $a$ by 0 on $W=$ $\left(W_{1} \times W_{2}\right) \cup W^{0}$. Since $W$ and $W^{0}$ are open neighborhoods of $\bar{T}$ and $T$ in $Y_{1} \times Y_{2}$, this defines a section of $\mathcal{B}_{T}$ over $U_{1} \times U_{2}$.

Now we consider $\left(X_{1}, Z_{1}, \sigma_{1}\right)$ and $\left(X_{2}, Z_{2}, \sigma_{2}\right)$ as at the beginning of this section. We set $X=X_{1} \times X_{2}, Z=Z_{1} \times Z_{2}, \sigma=\sigma_{1}+\sigma_{2}$. Then $(X, Z, \sigma)$ is also a kernel data satisfying (28). We keep the notations of diagram 30 and let $p: \tilde{X}_{Z} \rightarrow X$ be the projection. We also have a natural embedding $k: \tilde{X}_{Z} \rightarrow$ $\tilde{X}_{1} \times \tilde{X}_{2}$. We set $p^{\prime}=p_{1} \times p_{2}: \tilde{X}_{1} \times \tilde{X}_{2} \rightarrow X$.

Proposition 8.4. There exists a natural morphism $\mathcal{L}_{\sigma_{1}}^{\mathcal{A}} \underline{\mathcal{L}_{\sigma_{2}}^{\mathcal{A}}} \rightarrow \mathcal{L}_{\sigma}^{\mathcal{A}}$ in $\operatorname{Mod}\left(\mathcal{A}_{X_{1} \times X_{2}}\right)$.

Proof. We let $Q_{\sigma}^{0}$ be the image of $P_{\sigma}^{0}$ in $T_{Z_{1}} X_{1} \times T_{Z_{2}} X_{2} \subset \tilde{X}_{1} \times \tilde{X}_{2}$. We have a natural restriction morphism $k^{-1} \mathcal{B}_{Q_{\sigma}^{0}} \rightarrow \mathcal{B}_{P_{\sigma}^{0}}$ and Lemma 8.3 gives a morphism $\mathcal{B}_{P_{\sigma_{1}}^{0}} \otimes \mathcal{B}_{P_{\sigma_{2}}^{0}} \rightarrow \mathcal{B}_{Q_{\sigma}^{0}}$, so that we obtain $k^{-1}\left(\mathcal{B}_{P_{\sigma_{1}}^{0}} \otimes \mathcal{B}_{P_{\sigma_{2}}^{0}}\right) \rightarrow \mathcal{B}_{P_{\sigma}^{0}}$. We also have a morphism $k^{-1}\left(\mathbf{C}_{\Omega_{1}}^{\prime} \otimes \mathbf{C}_{\Omega_{2}}^{\prime}\right) \rightarrow \mathbf{C}_{\Omega}^{\prime}$. Now the proposition follows easily from these morphisms and the morphism of functors

$$
p_{1 ! !} \otimes p_{2 ! !} \rightarrow p_{! !}^{\prime} \rightarrow p_{! !}^{\prime} k_{! !} k^{-1}=p_{! !} k^{-1}
$$

\section{§9. Functorial properties of microlocalization}

In this section $f: X \rightarrow Y$ is a morphism of real analytic manifolds. We recall the functorial behavior of microlocalization and define the corresponding morphism for the microlocalization of $\mathcal{A}$-modules. This makes the link between kernels on 
$X \times \dot{T}^{*} X$ and $Y \times \dot{T}^{*} Y$ and we consider an intermediate space introduced in the diagram

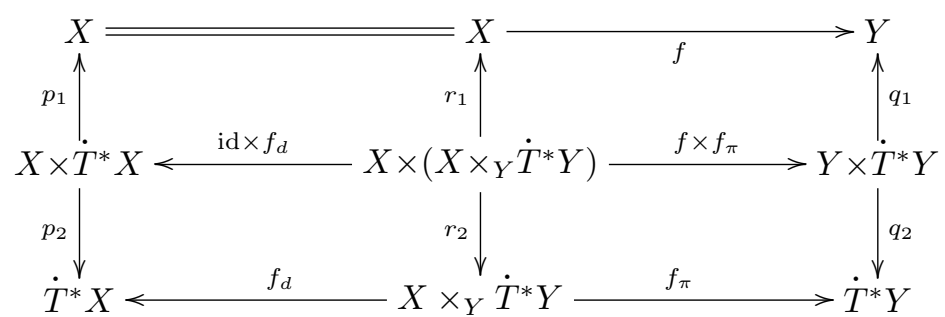

We define the submanifold $Z=X \times{ }_{Y} \dot{T}^{*} Y$ diagonally embedded in $X \times\left(X \times_{Y} \dot{T}^{*} Y\right)$ and the kernel data $\left(X \times\left(X \times_{Y} \dot{T}^{*} Y\right), Z, \sigma_{Y \leftarrow X}\right)$, where

$$
\sigma_{Y \leftarrow X}=\left(\operatorname{id} \times f_{d}\right)^{*}\left(\sigma_{X}\right)=\left(f \times f_{\pi}\right)^{*}\left(\sigma_{Y}\right) .
$$

This equality follows from $f_{d}^{*}\left(\omega_{X}\right)=f_{\pi}^{*}\left(\omega_{Y}\right)$. We note that $Z=\left(\operatorname{id} \times f_{d}\right)^{-1}\left(X \times_{X}\right.$ $\left.\dot{T}^{*} X\right)$ and $Z \subset\left(f \times f_{\pi}\right)^{-1}\left(Y \times_{Y} \dot{T}^{*} Y\right)$, with equality if $f$ is an embedding. This implies that hypothesis 228$)$ is satisfied for $\left(X \times\left(X \times_{Y} \dot{T}^{*} Y\right), Z, \sigma_{Y \leftarrow X}\right)$. We denote the corresponding kernel by $L_{Y \leftarrow X}=\mathcal{L}_{\sigma_{Y \leftarrow X}}$.

Inverse image. In the next proposition we assume that $f: X \rightarrow Y$ is an embedding. For $G \in \mathrm{D}^{+}\left(\mathbf{I}\left(\mathbf{C}_{Y}\right)\right)$ Theorem 2.4.4 of [9] gives a morphism

$$
\mathrm{R} f_{d ! !} f_{\pi}^{-1} \mu_{Y}(G) \rightarrow \mu_{X}\left(f^{-1} G\right) .
$$

Proposition 9.1. For an embedding $f: X \rightarrow Y$ and $G \in \operatorname{Mod}\left(\mathcal{A}_{Y}\right)$, we have a morphism of $\mathcal{A}_{T^{*} X}$-modules

$$
f_{d ! !} f_{\pi}^{*} \mu_{Y}^{\mathcal{A}}(G) \rightarrow \mu_{X}^{\mathcal{A}}\left(f^{*} G\right),
$$

whose image in $\mathrm{D}^{+}\left(\mathbf{I}\left(\mathbf{C}_{\dot{T}^{*} X}\right)\right)$ is morphism (34).

Proof. We first note the morphism of functors $f_{\pi}^{*} q_{2 ! !} \rightarrow r_{2 ! !}\left(f \times f_{\pi}\right)^{*}$. It is obtained by the following composition of adjunction morphisms, where we use the fact that $f$, hence $f_{\pi}$ and $f \times f_{\pi}$, are embeddings, so that direct and proper direct images coincide:

$$
f_{\pi}^{*} q_{2 ! !} \rightarrow f_{\pi}^{*} q_{2 ! !}\left(f \times f_{\pi}\right)_{*}\left(f \times f_{\pi}\right)^{*} \simeq f_{\pi}^{*} f_{\pi *} r_{2 ! !}\left(f \times f_{\pi}\right)^{*} \rightarrow r_{2 ! !}\left(f \times f_{\pi}\right)^{*} .
$$

We also note the morphisms between kernels:

$$
\left(f \times f_{\pi}\right)^{*} L_{Y}^{\mathcal{A}} \rightarrow L_{Y \leftarrow X}^{\mathcal{A}} \otimes \omega_{X \mid Y}^{\otimes-1}, \quad\left(\text { id } \times f_{d}\right)_{! !}\left(L_{Y \leftarrow X}^{\mathcal{A}} \otimes \omega_{X \mid Y}^{\otimes-1}\right) \rightarrow L_{X}^{\mathcal{A}} .
$$

The first one is morphism (32) applied to $f \times f_{\pi}$ : we note that $f \times f_{\pi}$ is clean with respect to $Y \times_{Y} T^{*} Y$ and $X \times_{Y} T^{*} Y=\left(f \times f_{\pi}\right)^{-1}\left(Y \times_{Y} T^{*} Y\right)$. The second one is 
morphism (31) applied to id $\times f_{d}$. Now the morphism of the proposition is defined by the succession of morphisms

$$
\begin{aligned}
f_{d ! !} f_{\pi}^{*} \mu_{Y}^{\mathcal{A}}(G) & =f_{d ! !} f_{\pi}^{*} q_{2 ! !}\left(L_{Y}^{\mathcal{A}} \otimes_{\mathcal{A}} q_{1}^{*} G\right) \\
& \rightarrow f_{d ! !} r_{2 ! !}\left(\left(f \times f_{\pi}\right)^{*} L_{Y}^{\mathcal{A}} \otimes_{\mathcal{A}} r_{1}^{*} f^{*} G\right) \\
& \longleftarrow p_{2 ! !}\left(\left(\mathrm{id} \times f_{d}\right) ! !\left(f \times f_{\pi}\right)^{*} L_{Y}^{\mathcal{A}} \otimes_{\mathcal{A}} p_{1}^{*} f^{*} G\right) \\
& \rightarrow p_{2 ! !}\left(L_{X}^{\mathcal{A}} \otimes_{\mathcal{A}} p_{1}^{*} f^{*} G\right),
\end{aligned}
$$

where in line (39) we used morphism (36) and the commutativity of inverse image and tensor product, and in (40) the identities $f_{d} r_{2}=p_{2}\left(\mathrm{id} \times f_{d}\right), r_{1}=p_{1}\left(\mathrm{id} \times f_{d}\right)$ and the projection formula for (id $\times f_{d}$ ) (Lemma 6.6). The last morphism is the composition of the morphisms in (37).

Direct image. Theorem 2.4.2 of [9] gives a morphism $\mathrm{R} f_{\pi ! !} f_{d}^{-1} \mu_{X}(F) \rightarrow \mu_{Y}\left(f_{! !} F\right)$ for $F \in \mathrm{D}^{b}\left(\mathbf{I}\left(\mathbf{C}_{X}\right)\right)$. We can define its analog for $\mathcal{A}$-modules if we assume that $f$ is a submersion and $F$ is of the type $F=f^{!} G \simeq f^{-1} G \otimes \omega_{X \mid Y}$; then the above morphism induces

$$
\mathrm{R} f_{\pi ! !} f_{d}^{-1} \mu_{X}\left(f^{-1} G \otimes \omega_{X \mid Y}\right) \rightarrow \mu_{Y}(G) .
$$

Proposition 9.2. There exists a natural morphism of $\mathcal{A}_{\dot{T}^{*} Y}$-modules, for a submersion $f: X \rightarrow Y$ and for $G \in \operatorname{Mod}\left(\mathcal{A}_{Y}\right)$,

$$
f_{\pi ! !} f_{d}^{*} \mu_{X}^{\mathcal{A}}\left(f^{*} G \otimes \omega_{X \mid Y}\right) \rightarrow \mu_{Y}^{\mathcal{A}}(G),
$$

whose image in $\mathrm{D}^{+}\left(\mathbf{I}\left(\mathbf{C}_{\dot{T}^{*} Y}\right)\right)$ is morphism 42 .

Proof. We set $F=f^{*} G \otimes \omega_{X \mid Y}$ and define (43) by the sequence of morphisms (with the notations of diagram 33 )

$$
\begin{aligned}
f_{\pi ! !} & f_{d}^{*} p_{2 ! !}\left(L_{X}^{\mathcal{A}} \otimes_{\mathcal{A}} p_{1}^{*} F\right) \\
& \rightarrow f_{\pi ! !} r_{2 ! !}\left(\left(\mathrm{id} \times f_{d}\right)^{*} L_{X}^{\mathcal{A}} \otimes r_{1}^{*} F\right) \\
& \simeq q_{2 ! !}\left(f \times f_{\pi}\right)_{! !}\left(\left(\mathrm{id} \times f_{d}\right)^{*} L_{X}^{\mathcal{A}} \otimes r_{1}^{-1} \omega_{X \mid Y} \otimes_{\mathcal{A}}\left(f \times f_{\pi}\right)^{*} q_{1}^{*} G\right) \\
& \leftarrow q_{2 ! !}\left(\left(f \times f_{\pi}\right)_{! !}\left(\left(\mathrm{id} \times f_{d}\right)^{*} L_{X}^{\mathcal{A}} \otimes r_{1}^{-1} \omega_{X \mid Y}\right) \otimes_{\mathcal{A}} q_{1}^{*} G\right) \\
& \rightarrow q_{2 ! !}\left(L_{Y}^{\mathcal{A}} \otimes_{\mathcal{A}} q_{1}^{*} G\right),
\end{aligned}
$$

where in line 45 we used the base change formula $f_{d}^{*} p_{2 ! !} \rightarrow r_{2 ! !}\left(\mathrm{id} \times f_{d}\right)^{*}$ and the identity $r_{1}=p_{1}\left(\mathrm{id} \times f_{d}\right)$, in line (46) the identities $f_{\pi} r_{2}=q_{2}\left(f \times f_{\pi}\right)$ and $f r_{1}=q_{1}\left(f \times f_{\pi}\right)$, and in line 477 the projection formula for $f \times f_{\pi}$. The last line is given by the composition of

$$
\left(\text { id } \times f_{d}\right)^{*} L_{X}^{\mathcal{A}} \rightarrow L_{Y \leftarrow X}^{\mathcal{A}} \quad \text { and } \quad\left(f \times f_{\pi}\right) ! !\left(L_{Y \leftarrow X}^{\mathcal{A}} \otimes r_{1}^{-1} \omega_{X \mid Y}\right) \rightarrow L_{Y}^{\mathcal{A}},
$$


which are given respectively by 32 and (31) (for the first morphism we note that id $\times f_{d}$ is transversal to $X \times_{X} T^{*} X$ and for the second one we note that the restriction of $r_{1}^{-1} \omega_{X \mid Y}$ to $X \times_{Y} T^{*} Y$ is isomorphic to $\left.\omega_{X \times_{Y} T^{*} Y \mid Y \times_{Y} T^{*} Y}\right)$.

External product. For $F \in \mathrm{D}^{+}\left(\mathbf{I}\left(\mathbf{C}_{X}\right)\right)$ and $G \in \mathrm{D}^{+}\left(\mathbf{I}\left(\mathbf{C}_{Y}\right)\right)$ Proposition 2.1.14 of [9] implies the existence of a natural morphism

$$
\mu_{X} F \otimes \mu_{Y} G \rightarrow \mu_{X \times Y}(F \otimes G) .
$$

Proposition 9.3. For $F \in \operatorname{Mod}\left(\mathcal{A}_{X}\right)$ and $G \in \operatorname{Mod}\left(\mathcal{A}_{Y}\right)$ there exists a natural morphism

$$
\left.\mu_{X}^{\mathcal{A}} F \underline{\otimes} \mu_{Y}^{\mathcal{A}} G \rightarrow \mu_{X \times Y}^{\mathcal{A}}(F \underline{\otimes} G)\right|_{\dot{T}^{*} X \times \dot{T}^{*} Y},
$$

whose image in $\mathrm{D}^{+}\left(\mathbf{I}\left(\mathbf{C}_{\dot{T}^{*} X \times \dot{T}^{*} Y}\right)\right)$ is $(49)$.

Proof. The existence of the morphism follows from the Künneth formula and Proposition 8.4. It coincides with the already known construction outside the zero section by Proposition 7.9 .

\section{§10. Composition of kernels}

We first recall some standard notations and definitions. We consider three analytic manifolds $X, Y, Z$ and we let $q_{i j}$ be the projection from $X \times Y \times Z$ to the $i^{\text {th }} \times j^{\text {th }}$ factors and $p_{i j}$ be the similar projection from $T^{*} X \times T^{*} Y \times T^{*} Z$. We also denote by $a: T^{*} Y \rightarrow T^{*} Y$ the antipodal map and we set $p_{12}^{a}=($ id $\times a) \circ p_{12}$. For $F \in \mathrm{D}^{+}\left(\mathbf{I}\left(\mathbf{C}_{X \times Y}\right)\right), G \in \mathrm{D}^{+}\left(\mathbf{I}\left(\mathbf{C}_{Y \times Z}\right)\right)$ and $\mathfrak{F} \in \mathrm{D}^{+}\left(\mathbf{I}\left(\mathbf{C}_{T^{*} X \times T^{*} Y}\right)\right), \mathfrak{G} \in$ $\mathrm{D}^{+}\left(\mathbf{I}\left(\mathbf{C}_{T^{*} Y \times T^{*} Z}\right)\right)$ we define

$$
F \circ G=\mathrm{R} q_{13 ! !}\left(q_{12}^{-1} F \otimes q_{23}^{-1} G\right), \quad \mathfrak{F}^{a} \circ \mathfrak{G}=\mathrm{R} p_{13 ! !}\left(p_{12}^{a-1} \mathfrak{F} \otimes p_{23}^{-1} \mathfrak{G}\right) .
$$

We set for short $M=X \times Y \times Y \times Z, N=X \times Y \times Z$ and let $j: N \rightarrow M$ be the diagonal embedding. We define the maps

$$
\begin{array}{ll}
k: T^{*} N \hookrightarrow N \times_{M} T^{*} M, & (x, y, z ; \xi, \eta, \zeta) \mapsto(x, y, y, z ; \xi,-\eta, \eta, \zeta), \\
\tau: T^{*} N \rightarrow N \times_{X \times Z} T^{*}(X \times Z), & (x, y, z ; \xi, \eta, \zeta) \mapsto(x, y, z ; \xi, \zeta), \\
p=j_{\pi} \circ k, &
\end{array}
$$


and obtain the following commutative diagram with a Cartesian square:

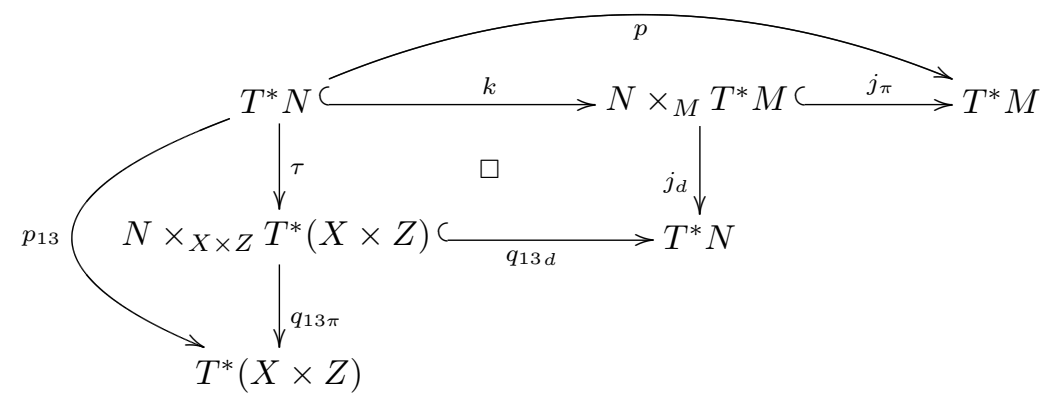

We note that $\mathfrak{F}^{\circ} \stackrel{a}{\circ} \simeq \mathrm{R} p_{13 ! !} p^{-1}(\mathfrak{F} \otimes \mathfrak{G})$. Theorem 2.5.1 of [9] gives a natural morphism, for $K_{1} \in \mathrm{D}^{+}\left(\mathbf{I}\left(\mathbf{C}_{X \times Y}\right)\right), K_{2} \in \mathrm{D}^{+}\left(\mathbf{I}\left(\mathbf{C}_{Y \times Z}\right)\right)$,

$$
\mu_{X \times Y} K_{1} \stackrel{a}{\circ} \mu_{Y \times Z} K_{2} \rightarrow \mu_{X \times Z}\left(K_{1} \circ K_{2}\right) .
$$

It can be restated as follows. For complexes $K_{1} \in \mathrm{D}^{+}\left(\mathbf{I}\left(\mathbf{C}_{X \times Y}\right)\right), K_{2} \in$ $\mathrm{D}^{+}\left(\mathbf{I}\left(\mathbf{C}_{Y \times Z}\right)\right)$ and $K_{3} \in \mathrm{D}^{+}\left(\mathbf{I}\left(\mathbf{C}_{X \times Z}\right)\right)$, with a morphism $q_{12}^{-1} K_{1} \otimes q_{23}^{-1} K_{2} \rightarrow$ $q_{13}^{-1} K_{3} \otimes \omega_{Y}$, there exists a natural morphism

$$
\mu_{X \times Y} K_{1} \stackrel{a}{\circ} \mu_{Y \times Z} K_{2} \rightarrow \mu_{X \times Z} K_{3} .
$$

This is equivalent to (53): indeed using the adjunction between $\mathrm{R} q_{13}$ !! and $q_{13}^{!}$we may apply (54) to $K_{3}=K_{1} \circ K_{2}$ and recover (53). But for $\mathcal{A}$-modules we do not have this adjunction and the statement of the following proposition is actually weaker than an $\mathcal{A}$-module analog of (53).

For $\mathfrak{F} \in \operatorname{Mod}\left(\mathcal{A}_{T^{*} X \times T^{*} Y}\right)$ and $\mathfrak{G} \in \operatorname{Mod}\left(\mathcal{A}_{T^{*} Y \times T^{*} Z}\right)$ we set

$$
\mathfrak{F}^{a \mathcal{A}} \mathfrak{G}=p_{13 ! !}\left(p_{12}^{a *} \mathfrak{F} \otimes_{\mathcal{A}_{N}} p_{23}^{*} \mathfrak{G}\right) \simeq p_{13 ! !} p^{*}(\mathfrak{F} \unrhd \mathfrak{G}) .
$$

If $\mathfrak{F}$, resp. $\mathfrak{G}$, is defined only on $\dot{T}^{*}(X \times Y)$, resp. $\dot{T}^{*}(Y \times Z)$, then $\mathfrak{F}^{a \mathcal{A}} \mathfrak{G}$ is defined on $\dot{T}^{*} X \times \dot{T}^{*} Z$. In $\mathrm{D}^{+}\left(\mathbf{I}\left(\mathbf{C}_{T^{*} X \times T^{*} Z}\right)\right)$ we have the morphisms

$$
\mathfrak{F}^{a} \circ \mathfrak{G} \rightarrow \mathrm{R} p_{13 ! !}\left(p_{12}^{a *} \mathfrak{F} \otimes_{\mathcal{A}_{N}} p_{23}^{*} \mathfrak{G}\right) \longleftarrow \mathfrak{F} \stackrel{a \mathcal{A}}{\circ} \mathfrak{G},
$$

where the second arrow is an isomorphism by Proposition 6.5 .

Proposition 10.1. For $K_{1} \in \operatorname{Mod}\left(\mathcal{A}_{X \times Y}\right), K_{2} \in \operatorname{Mod}\left(\mathcal{A}_{Y \times Z}\right)$ and $K_{3} \in$ $\operatorname{Mod}\left(\mathcal{A}_{X \times Z}\right)$ with a morphism $q_{12}^{*} K_{1} \otimes_{\mathcal{A}} q_{23}^{*} K_{2} \rightarrow q_{13}^{*} K_{3} \otimes \omega_{Y}$ there exists a natural morphism in $\operatorname{Mod}\left(\mathcal{A}_{\dot{T}^{*} Y \times \dot{T}^{*} Z}\right)$,

$$
\mu_{X \times Y}^{\mathcal{A}} K_{1} \stackrel{a \mathcal{A}}{\circ} \mu_{Y \times Z}^{\mathcal{A}} K_{2} \rightarrow \mu_{X \times Z}^{\mathcal{A}} K_{3},
$$


which makes a commutative diagram in $\mathrm{D}^{+}\left(\mathbf{I}\left(\mathbf{C}_{\left.\dot{T}^{*} X \times \dot{T}^{*} Z\right)}\right)\right)$ with (54),

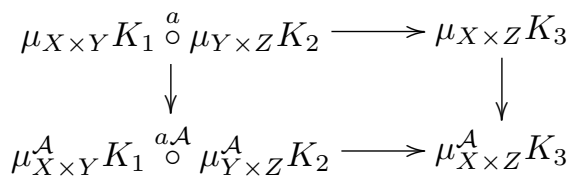

Proof. By definition $\mu_{X \times Y}^{\mathcal{A}} K_{1} \stackrel{a \mathcal{A}}{\circ} \mu_{Y \times Z}^{\mathcal{A}} K_{2}=p_{13 ! !} p^{*}\left(\mu_{X \times Y}^{\mathcal{A}} K_{1} \underline{\otimes} \mu_{Y \times Z} K_{2}\right)$. The external tensor product (50) gives $\mu_{X \times Y}^{\mathcal{A}} K_{1} \unrhd \mu_{Y \times Z}^{\mathcal{A}} K_{2} \rightarrow \mu_{M}^{\mathcal{A}}\left(K_{1} \unrhd K_{2}\right)$ and the base change formula (21) gives $p_{13 ! !} p^{*}=q_{13 \pi ! !} \tau_{! !} k^{*} j_{\pi}^{*} \longleftarrow q_{13 \pi ! !} q_{13 d}^{*} j_{d ! !} j_{\pi}^{*}$, which is an isomorphism because $q_{13 d}$ is an embedding and $j_{d}$ is smooth. We obtain the morphisms

$$
\begin{aligned}
\mu_{X \times Y}^{\mathcal{A}} K_{1} \stackrel{a \mathcal{A}}{\circ} \mu_{Y \times Z}^{\mathcal{A}} K_{2} & \rightarrow q_{13 \pi ! !} q_{13 d}^{*} j_{d ! !} j_{\pi}^{*}\left(\mu_{M}^{\mathcal{A}}\left(K_{1} \underline{\otimes} K_{2}\right)\right) \\
& \rightarrow q_{13 \pi ! !} q_{13 d}^{*} \mu_{N}^{\mathcal{A}} j^{*}\left(K_{1} \underline{\otimes} K_{2}\right) \\
& \rightarrow q_{13 \pi ! !} q_{13 d}^{*} \mu_{N}^{\mathcal{A}}\left(q_{13}^{*} K_{3} \otimes \omega_{Y}\right) \\
& \rightarrow \mu_{X \times Z}^{\mathcal{A}} K_{3}
\end{aligned}
$$

where in the second line we have applied Proposition 9.1. in the third the hypothesis and in the fourth Proposition 9.2

We are in fact only interested in the following example. We assume now that $X, Y, Z$ are complex analytic manifolds. We use the $\mathcal{A}$-module $\mathbb{O}_{X}$ and its variants introduced in Definition 6.8. We set $K_{1}=\mathbb{O}_{X \times Y}^{\left(0, d_{Y}^{c}\right)}\left[d_{Y}^{c}\right]$, which gives a resolution of $\mathcal{O}_{X \times Y}^{t\left(0, d_{Y}^{c}\right)}\left[d_{Y}^{c}\right], K_{2}=\mathbb{O}_{Y \times Z}^{\left(0, d_{Z}^{c}\right)}\left[d_{Z}^{c}\right], K_{3}=\mathbb{O}_{X \times Z}^{\left(0, d_{Z}^{c}\right)}\left[d_{Z}^{c}\right]$. With these notations morphism 27] yields a morphism $q_{12}^{*} K_{1} \otimes_{\mathcal{A}} q_{23}^{*} K_{2} \rightarrow q_{13}^{*} K_{3} \otimes \omega_{Y}$ and Proposition 10.1 gives the microlocal convolution

$$
\mu_{X \times Y}^{\mathcal{A}} \mathbb{O}_{X \times Y}^{\left(0, d_{Y}^{c}\right)}\left[d_{Y}^{c}\right] \stackrel{a \mathcal{A}}{\circ} \mu_{Y \times Z}^{\mathcal{A}} \mathbb{O}_{Y \times Z}^{\left(0, d_{Z}^{c}\right)}\left[d_{Z}^{c}\right] \rightarrow \mu_{X \times Z}^{\mathcal{A}} \mathbb{O}_{X \times Z}^{\left(0, d_{Z}^{c}\right)}\left[d_{Z}^{c}\right] .
$$

This convolution product is associative, because the composition of kernels $\begin{gathered}a \mathcal{A} \\ 0\end{gathered}$ is associative as also is the integration morphism by Fubini.

We will in fact use morphism (56) in a slightly more general situation, namely for complexes of the type $\mathcal{H} \operatorname{om}\left(\pi^{-1} F, \mu G\right)$, rather than $\mu G$. We first introduce notations in the category of complexes:

$$
F \stackrel{0}{\circ} G=q_{13 ! !}\left(q_{12}^{-1} F \otimes q_{23}^{-1} G\right), \quad \mathfrak{F} \stackrel{a 0}{\circ} \mathfrak{G}=p_{13 ! !}\left(p_{12}^{a-1} \mathfrak{F} \otimes p_{23}^{-1} \mathfrak{G}\right) .
$$

Proposition 10.2. For $F \in \mathrm{C}^{+}\left(\mathbf{C}_{(X \times Y)_{s a}}\right), \quad G \in \mathrm{C}^{+}\left(\mathbf{C}_{(Y \times Z)_{s a}}\right)$, $\mathfrak{F} \in$ $\operatorname{Mod}\left(\mathcal{A}_{T^{*}(X \times Y)}\right)$ and $\mathfrak{G} \in \operatorname{Mod}\left(\mathcal{A}_{T^{*}(Y \times Z)}\right)$ we have a natural morphism

$$
\mathcal{H o m}\left(\pi_{X \times Y}^{-1} F, \mathfrak{F}\right) \stackrel{a \mathcal{A}}{\circ} \mathcal{H o m}\left(\pi_{Y \times Z}^{-1} G, \mathfrak{G}\right) \rightarrow \mathcal{H o m}\left(\pi_{X \times Z}^{-1}\left(F^{\circ}{ }^{\circ} G\right), \mathfrak{F}^{a \mathcal{A}} \mathfrak{G}\right) .
$$


Proof. We keep the notations of diagram (52). Let us denote by LHS the left hand side of (58). We have the morphisms

$$
\begin{aligned}
\mathrm{LHS} & =p_{13 ! !} p^{*}\left(\mathcal{H o m}\left(\pi_{X \times Y}^{-1} F, \mathfrak{F}\right) \underline{\boldsymbol{H}} \mathcal{H o m}\left(\pi_{Y \times Z}^{-1} G, \mathfrak{G}\right)\right) \\
& \simeq p_{13 ! !} p^{*} \mathcal{H o m}\left(\pi_{M}^{-1}(F \otimes G), \mathfrak{F} \underline{\otimes} \mathfrak{G}\right) \\
& \rightarrow \mathcal{H o m}\left(p_{13 *} p^{-1} \pi_{M}^{-1}(F \otimes G), \mathfrak{F} \circ \mathfrak{\mathcal { A }}\right),
\end{aligned}
$$

where in the last line we have used the morphisms of functors $p^{*} \mathcal{H} \operatorname{om}(\cdot, \cdot) \rightarrow$ $\mathcal{H o m}\left(p^{-1}(\cdot), p^{*}(\cdot)\right)$ and $p_{13 ! !} \mathcal{H o m}(\cdot, \cdot) \rightarrow \mathcal{H o m}\left(p_{13 *}(\cdot), p_{13 ! !}(\cdot)\right)$.

We let $\sigma: N \times_{X \times Z} T^{*}(X \times Z) \rightarrow T^{*} N$ be induced by the inclusion of the zero section of $Y$ and we let $\pi_{N}^{\prime}: N \times_{X \times Z} T^{*}(X \times Z) \rightarrow N$ be the projection. Then $\pi_{M} \circ p \circ \sigma=j \circ \pi_{N}^{\prime}$. Moreover, since we deal with conic sheaves we have the isomorphism of functors $\tau_{*} \simeq \sigma^{-1}$. We also have a morphism $q_{13 \pi ! !} \rightarrow q_{13 \pi *}$. We deduce the sequence of morphisms

$$
\begin{aligned}
p_{13 *} p^{-1} \pi_{M}^{-1} \simeq q_{13 \pi *} & \tau_{*} p^{-1} \pi_{M}^{-1} \\
& \leftarrow q_{13 \pi ! !} \sigma^{-1} p^{-1} \pi_{M}^{-1} \simeq q_{13 \pi ! !} \pi_{N}^{\prime-1} j^{-1} \simeq \pi_{X \times Z}^{-1} q_{13 ! !} j^{-1},
\end{aligned}
$$

where the last isomorphism is a base change. So we obtain $\pi_{X \times Z}^{-1} q_{13 ! !} j^{-1} \rightarrow$ $p_{13 *} p^{-1} \pi_{M}^{-1}$ and composing this morphism with (59) we deduce 58).

Remark 10.3. We can define analogs of morphism $\sqrt{58}$ in the derived category or in the category of complexes. We then obtain the first two lines in the commutative diagram

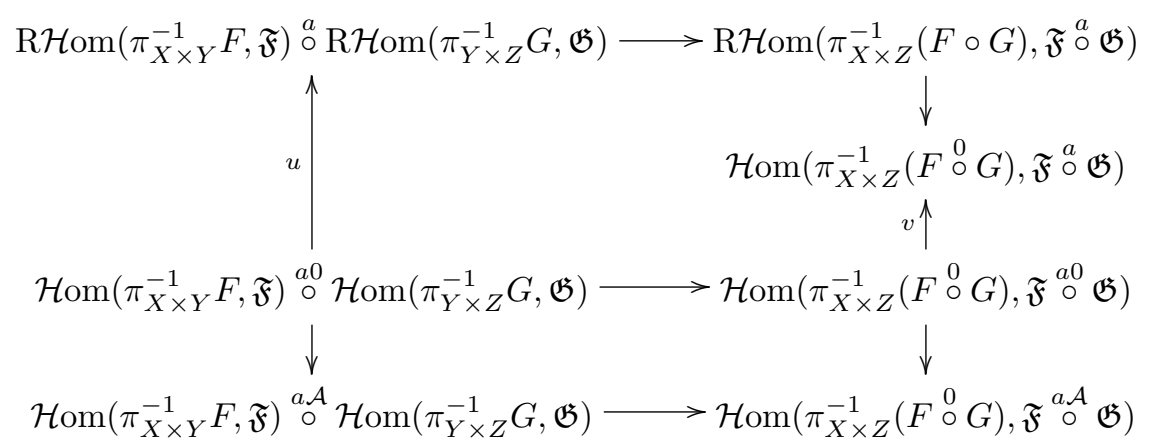

The vertical arrows $u$ and $v$ in this diagram go in the wrong direction but it will be used in cases where they are isomorphisms.

\section{§11. $\mathcal{E}$-modules}

In this section $X$ is a complex analytic manifold of complex dimension $n=d_{X}^{c}$ and $\Delta$ denotes the diagonal of $X \times X$. We identify $T^{*} X$ and $T_{\Delta}^{*}(X \times X)$ by the 
first projection. We denote by $\mathcal{E}_{X}$ the sheaf of microdifferential operators of finite order; this is a sheaf on $T^{*} X$. It can be defined by a construction similar to Sato's definition of microdifferential operators of infinite order, using the tempered microlocalization; this is done in [1] (see also [1]-here we can use (2) as a definition of $T$ - $\mu$ hom), as follows. We let $\gamma: T^{*} X \rightarrow T^{*} X / \mathbf{C}^{*}$ be the quotient map by the action of $\mathbf{C}^{*}$ in the fibers. Then $\mathcal{E}_{X} \simeq \gamma^{-1} \gamma_{*}\left(\mathcal{E}_{X}^{\mathbf{R}, f}\right)$ where $\mathcal{E}_{X}^{\mathbf{R}, f}$ is the sheaf on $T^{*} X \simeq T_{\Delta}^{*}(X \times X)$,

$$
\mathcal{E}_{X}^{\mathbf{R}, f}=T-\mu h o m\left(\mathbf{C}_{\Delta}, \mathcal{O}_{X \times X}^{(0, n)}[n]\right) .
$$

The product of $\mathcal{E}_{X}^{\mathbf{R}, f}$ is defined in [1] by the convolution product for tempered microlocalization. This can also be defined in the language of ind-sheaves, following [9]. We first define $\mathcal{E}_{X}^{\text {ind }} \in \mathrm{D}^{b}\left(\mathbf{I}\left(\mathbf{C}_{T^{*}(X \times X)}\right)\right)$ by

$$
\mathcal{E}_{X}^{\text {ind }}=\operatorname{R} \mathcal{I} \mathcal{H} \text { om }\left(\pi^{-1} \mathbf{C}_{\Delta}, \mu_{X \times X} \mathcal{O}_{X \times X}^{t(0, n)}[n]\right)
$$

where $\mathcal{O}_{X \times X}^{t(0, n)}$, defined in $(23)$ as an object of $\mathrm{D}^{b}\left(\mathbf{C}_{(X \times X)_{s a}}\right)$, is now considered in $\mathrm{D}^{b}\left(\mathbf{I}\left(\mathbf{C}_{X \times X}\right)\right)$ using the functor $I_{\tau}$. Thus $\mathcal{E}_{X}^{\text {ind }}$ has support on $T^{*} X \simeq T_{\Delta}^{*}(X \times X)$ but this does not imply that it is the image of an ind-sheaf on $T^{*} X$. We recall the notations $p_{1}, p_{2}: T^{*}(X \times X) \rightarrow T^{*} X$ for the projections, $a: T^{*} X \rightarrow T^{*} X$ for the antipodal map and we define the embedding

$$
\delta^{\prime}: T^{*} X \simeq T_{\Delta}^{*}(X \times X) \rightarrow T^{*}(X \times X), \quad(x, \xi) \mapsto(x, x, \xi,-\xi) .
$$

Since $\operatorname{supp} \mathcal{E}_{X}^{\text {ind }}=T_{\Delta}^{*}(X \times X)$ the morphisms of functors $p_{1 *} \rightarrow p_{1 *} \delta_{*}^{\prime} \delta^{\prime-1}=\delta^{\prime-1}$ and $p_{2 *} \rightarrow a^{-1} \delta^{\prime-1}$ induce isomorphisms:

$$
\delta^{\prime-1} \mathcal{E}_{X}^{\text {ind }} \simeq p_{1 *} \mathcal{E}_{X}^{\text {ind }} \simeq a^{-1} p_{2 *} \mathcal{E}_{X}^{\text {ind }}
$$

We could write the same isomorphisms with $p_{i ! !}, \mathrm{R} p_{i *}$ or $\mathrm{R} p_{i ! !}$ instead of $p_{i *}$.

Definition 11.1. We let $\mathcal{E}_{X}^{\text {ind }} \in \mathrm{D}^{b}\left(\mathbf{I}\left(\mathbf{C}_{T^{*} X}\right)\right)$ be the ind-sheaf on $T^{*} X$ defined by 61 .

Since the functor $\alpha$ from ind-sheaves to sheaves commutes with direct image and inverse image we have $\mathcal{E}_{X}^{\mathbf{R}, f} \simeq \alpha_{T^{*} X}\left(\mathcal{E}_{X}^{\text {ind }}\right)$. The complex $\mathcal{E}_{X}^{\text {ind }}$ comes equipped with a product in the sense of Definition 3.1, defined as follows (omitting the subscript in $\mu)$ :

(i) Using 61 we see that $\mathcal{E}_{X}^{\text {ind }} \otimes \mathcal{E}_{X}^{\text {ind }} \simeq \delta^{\prime-1}\left(\mathcal{E}_{X}^{\text {ind }} \stackrel{a}{\circ} \mathcal{E}_{X}^{\text {ind }}\right)$.

(ii) We have $\mathbf{C}_{\Delta} \circ \mathbf{C}_{\Delta}=\mathbf{C}_{\Delta}$ and the top line in diagram 60 with $X=Y=Z$ gives $\mathcal{E}_{X}^{\text {ind }} \otimes \mathcal{E}_{X}^{\text {ind }} \rightarrow \delta^{\prime-1} \mathrm{R} \mathcal{I} \mathcal{H}$ om $\left(\pi^{-1} \mathbf{C}_{\Delta}, \mu \mathcal{O}_{X \times X}^{t(0, n)} \stackrel{a}{\circ} \mu \mathcal{O}_{X \times X}^{t(0, n)}[2 n]\right)$.

(iii) Morphisms 260 and (54) give $\mu \mathcal{O}_{X \times X}^{t(0, n)} \stackrel{a}{\circ} \mu \mathcal{O}_{X \times X}^{t(0, n)}[2 n] \rightarrow \mu \mathcal{O}_{X \times X}^{t(0, n)}[n]$. 
The composition of (i)-(iii) defines the product $\mathcal{E}_{X}^{\text {ind }} \otimes \mathcal{E}_{X}^{\text {ind }} \rightarrow \mathcal{E}_{X}^{\text {ind }}$. The same discussion, applied to $X=Y$ and $Z$ a point, ends up with an action of $\mathcal{E}_{X}^{\text {ind }}$ on $\mu \mathcal{O}_{X}^{t}$ in the sense of Definition 3.1. We deduce an action of $\mathcal{E}_{X}^{\text {ind }}$ on $\operatorname{RIH} \mathcal{H}$ om $\left(\pi^{-1} F, \mu \mathcal{O}_{X}^{t}\right)$ for any $F \in \mathrm{D}^{b}\left(\mathbf{I}\left(\mathbf{C}_{X}\right)\right)$.

This product and this action are just morphisms in the derived category and do not endow the complex $\mathcal{E}_{X}^{\text {ind }}$ with a structure of algebra. However, when we go back to the derived category of sheaves with the functor $\alpha_{T^{*} X}$, the product gives a morphism $\mathcal{E}_{X}^{\mathbf{R}, f} \otimes \mathcal{E}_{X}^{\mathbf{R}, f} \rightarrow \mathcal{E}_{X}^{\mathbf{R}, f}$ which endows $\mathcal{E}_{X}^{\mathbf{R}, f}$ with a structure of algebra because $\mathcal{E}_{X}^{\mathbf{R}, f}$ is a sheaf (i.e. concentrated in degree 0 ). But this is not enough to de-

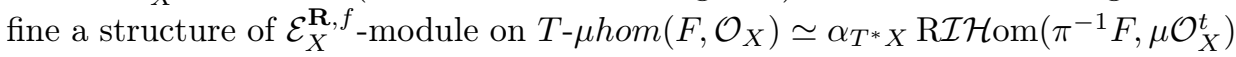
which is in general not concentrated in one degree.

To solve this problem we define a dg-algebra $\mathcal{E}_{X}^{\mathcal{A}}$ on the site $X_{s a}$ (and not merely an object in the derived category) such that $\mathcal{E}_{X}^{\text {ind }} \simeq I_{\tau}\left(\mathcal{E}_{X}^{\mathcal{A}}\right)$. We also define in the same way a dg-module over $\mathcal{E}_{X}^{\mathcal{A}}$ representing $\mu \mathcal{O}_{X}^{t}$. In fact our definition is exactly the previous one but in categories of $\mathcal{A}$-modules instead of derived categories.

Definition 11.2. We define a complex of sheaves on $\dot{T}^{*} X_{s a}$,

$$
\mathcal{E}_{X}^{\mathcal{A}}=\delta^{\prime-1} \mathcal{H} \operatorname{om}\left(\pi^{-1} \mathbf{C}_{\Delta}, \mu_{X \times X}^{\mathcal{A}} \mathbb{O}_{X \times X}^{(0, n)}[n]\right),
$$

with a product, $\mathcal{E}_{X}^{\mathcal{A}} \otimes \mathcal{E}_{X}^{\mathcal{A}} \rightarrow \mathcal{E}_{X}^{\mathcal{A}}$, defined as the composition of

$$
\mathcal{E}_{X}^{\mathcal{A}} \otimes \mathcal{E}_{X}^{\mathcal{A}} \rightarrow \delta^{\prime-1} \mathcal{H o m}\left(\pi^{-1} \mathbf{C}_{\Delta}, \mu^{\mathcal{A}} \mathbb{O}_{X \times X}^{(0, n)} \stackrel{a \mathcal{A}}{\circ} \mu^{\mathcal{A}} \mathbb{O}_{X \times X}^{(0, n)}[2 n]\right)
$$

and

$$
\mu^{\mathcal{A}} \mathbb{O}_{X \times X}^{(0, n)} \stackrel{a \mathcal{A}}{\circ} \mu^{\mathcal{A}} \mathbb{O}_{X \times X}^{(0, n)}[2 n] \rightarrow \mu^{\mathcal{A}} \mathbb{O}_{X \times X}^{(0, n)}[n],
$$

which are respectively given by (58) and (56) together with (27).

We also have a natural morphism $\mathcal{E}_{X}^{\mathcal{A}} \otimes \mu_{X}^{\mathcal{A}} \mathbb{O}_{X} \rightarrow \mu_{X}^{\mathcal{A}} \mathbb{O}_{X}$ defined like the above action of $\mathcal{E}_{X}^{\text {ind }}$ on $\mu \mathcal{O}_{X}^{t}$.

Proposition 11.3. The morphisms introduced in the previous definition give $\mathcal{E}_{X}^{\mathcal{A}}$ a structure of dg-algebra and give $\mu_{X}^{\mathcal{A}} \mathbb{O}_{X}$ a structure of $d g-\mathcal{E}_{X}^{\mathcal{A}}$-module.

Over $\dot{T}^{*} X$, we have isomorphisms $\mathcal{E}_{X}^{\text {ind }} \simeq I_{\tau}\left(\mathcal{E}_{X}^{\mathcal{A}}\right)$ and $\mu \mathcal{O}_{X}^{t} \simeq I_{\tau}\left(\mu_{X}^{\mathcal{A}} \mathbb{O}_{X}\right)$. Through these isomorphisms the product of $\mathcal{E}_{X}^{\mathcal{A}}$ and its action on $\mu_{X}^{\mathcal{A}} \mathbb{O}_{X}$ coincide with the product of $\mathcal{E}_{X}^{\text {ind }}$ and its action on $\mu \mathcal{O}_{X}^{t}$ defined above.

Proof. The complex $\mathcal{E}_{X}^{\mathcal{A}}$ is a dg-algebra and $\mu_{X}^{\mathcal{A}} \mathbb{O}_{X}$ is a dg- $\mathcal{E}_{X}^{\mathcal{A}}$-module because the product and the action are defined in categories of complexes and not merely up to homotopy. 
The second part of the proposition follows from Proposition 10.1 and Remark 10.3. With the notations of diagram 60 we are interested in the cases $\mathfrak{F}=\mu_{X}^{\mathcal{A}} \mathbb{O}_{X \times X}^{(0, n)}[n], F=\mathbf{C}_{\Delta}$ and either $\mathfrak{G}=\mathfrak{F}, G=F$ (for the product) or $\mathfrak{G}=\mu_{X}^{\mathcal{A}} \mathbb{O}_{X}, G=\mathbf{C}_{X}$ (for the action).

By Proposition 10.1, using the composition of kernels $\mathfrak{F} \stackrel{a}{\circ} \mathfrak{G} \rightarrow \mathfrak{G}$ and its analogs for $\stackrel{a 0}{\circ}$ and $\stackrel{a \mathcal{A}}{\circ}$ we change diagram 60 into another commutative diagram, where $\mathfrak{F} \stackrel{a}{\circ} \mathfrak{G}$ is replaced by $\mathfrak{G}$. We just have to check that the vertical arrows $u$ and $v$ (in the notations of (60) ) in this new diagram are isomorphisms.

By Proposition 7.9 $\mathfrak{F}$ and $\mathfrak{G}$ consist of quasi-injective sheaves on $T^{*} X_{s a}$ and so are acyclic for the functors $\mathcal{H} \operatorname{om}(H, \cdot), H$ constructible. In particular the $\mathcal{H}$ om sheaves in diagram 60 are isomorphic to the RHom. For the composition of kernels o we also have to compute a direct image. Since we deal with $\mathcal{A}$-modules Proposition 6.5 implies that direct images and derived direct images coincide. This proves that the vertical arrows are isomorphisms.

We still have to make the link between $\mathcal{E}_{X}^{\mathcal{A}}$ and $\mathcal{E}_{X}^{\mathbf{R}, f}$. We note that $\rho^{-1} \mathcal{E}_{X}^{\mathcal{A}}$ is quasi-isomorphic to $\mathcal{E}_{X}^{\mathbf{R}, f}$. In particular $\rho^{-1} \mathcal{E}_{X}^{\mathcal{A}}$ has its cohomology concentrated in degree 0 and we have isomorphisms of sheaves

$$
\mathcal{E}_{X}^{\mathbf{R}, f} \simeq H^{0}\left(\rho^{-1} \mathcal{E}_{X}^{\mathcal{A}}\right) \simeq H^{0}\left(\alpha I_{\tau}\left(\mathcal{E}_{X}^{\mathcal{A}}\right)\right)
$$

Moreover the structure of dg-algebra on $\mathcal{E}_{X}^{\mathcal{A}}$ gives a structure of dg-algebra on $\rho^{-1} \mathcal{E}_{X}^{\mathcal{A}}$ and a structure of algebra on $H^{0}\left(\rho^{-1} \mathcal{E}_{X}^{\mathcal{A}}\right)$. The above proposition implies that this product induced on $\mathcal{E}_{X}^{\mathbf{R}, f}$ coincides with the usual one.

We also have a structure of $\mathrm{dg}-\mathcal{E}_{X}^{\mathcal{A}}$-module on $\mu_{X}^{\mathcal{A}} \mathbb{O}_{X}$; in particular it defines an object $I_{\tau}\left(\mu_{X}^{\mathcal{A}} \mathbb{O}_{X}\right) \in \mathrm{D}\left(I_{\tau}\left(\mathcal{E}_{X}^{\mathcal{A}}\right)\right)$. For any $G \in \mathrm{D}^{-}\left(\mathbf{I}\left(\mathbf{C}_{T^{*} X}\right)\right)$ the complex $\operatorname{RIH} \operatorname{Hom}\left(G, I_{\tau}\left(\mu_{X}^{\mathcal{A}} \mathbb{O}_{X}\right)\right)$ is thus also naturally defined as an object of $\mathrm{D}\left(I_{\tau}\left(\mathcal{E}_{X}^{\mathcal{A}}\right)\right)$. For $G=\pi^{-1} F, F \in \mathrm{D}^{-}\left(\mathbf{I}\left(\mathbf{C}_{X}\right)\right)$, we deduce that

$$
T-\mu h o m\left(F, \mathcal{O}_{X}\right)=\alpha \operatorname{RIH} \mathcal{H o m}\left(\pi^{-1} F, I_{\tau}\left(\mu_{X}^{\mathcal{A}} \mathbb{O}_{X}\right)\right) \in \mathrm{D}\left(\rho^{-1} \mathcal{E}_{X}^{\mathcal{A}}\right),
$$

and, by construction, the corresponding action in $\mathrm{D}\left(\mathbf{C}_{T^{*} X}\right)$

$$
\rho^{-1} \mathcal{E}_{X}^{\mathcal{A}} \otimes T-\mu h o m\left(F, \mathcal{O}_{X}\right) \rightarrow T-\mu h o m\left(F, \mathcal{O}_{X}\right)
$$

coincides with the action of $\mathcal{E}_{X}^{\mathbf{R}, f}$ on $T$ - $\mu h o m\left(F, \mathcal{O}_{X}\right)$ defined above.

Thus we are almost done, except that $T$ - $\mu$ hom $\left(F, \mathcal{O}_{X}\right)$ is defined as an object of $\mathrm{D}\left(\rho^{-1} \mathcal{E}_{X}^{\mathcal{A}}\right)$ rather than $\mathrm{D}\left(\mathcal{E}_{X}^{\mathbf{R}, f}\right)$. But the dg-algebra $\rho^{-1} \mathcal{E}_{X}^{\mathcal{A}}$ is quasi-isomorphic to $\mathcal{E}_{X}^{\mathbf{R}, f}$ and it just remains to apply Corollary 3.3 as follows. We have the quasiisomorphisms of dg-algebras on $\dot{T}^{*} X$

$$
\rho^{-1} \mathcal{E}_{X}^{\mathcal{A}} \stackrel{\phi_{\leq 0}}{\longleftarrow} \tau_{\leq 0} \rho^{-1} \mathcal{E}_{X}^{\mathcal{A}} \stackrel{\phi_{0}}{\longrightarrow} \mathcal{E}_{X}^{\mathbf{R}, f}
$$


and the equivalence of categories $\phi_{0}^{*} \circ \phi_{\leq 0 *}: \mathrm{D}\left(\rho^{-1} \mathcal{E}_{X}^{\mathcal{A}}\right) \simeq \mathrm{D}\left(\mathcal{E}_{X}^{\mathbf{R}, f}\right)$. We set $\mathcal{E}_{X}^{\prime}=$ $\beta_{T^{*} X}\left(\rho^{-1} \mathcal{E}_{X}^{\mathcal{A}}\right)$ so that we have an adjunction morphism $\mathcal{E}_{X}^{\prime} \rightarrow I_{\tau}\left(\mathcal{E}_{X}^{\mathcal{A}}\right)$. This morphism induces the restriction of scalars $r: \mathrm{D}\left(I_{\tau}\left(\mathcal{E}_{X}^{\mathcal{A}}\right)\right) \rightarrow \mathrm{D}\left(\mathcal{E}_{X}^{\prime}\right)$ and $\phi_{0}^{*} \circ \phi_{\leq 0 *}$ induces an equivalence of categories $\Phi: \mathrm{D}\left(\mathcal{E}_{X}^{\prime}\right) \simeq \mathrm{D}\left(\beta_{T^{*} X}\left(\mathcal{E}_{X}^{\mathbf{R}, f}\right)\right)$. Hence we obtain an object

$$
\mathcal{O}_{X}^{\mu}=\Phi\left(r\left(I_{\tau}\left(\mu_{X}^{\mathcal{A}} \mathbb{O}_{X}\right)\right)\right) \in \mathrm{D}\left(\beta_{T^{*} X}\left(\mathcal{E}_{X}^{\mathbf{R}, f}\right)\right)
$$

representing $\mu \mathcal{O}_{X}^{t}$ and we can state the final result:

Theorem 11.4. The object $\mathcal{O}_{X}^{\mu} \in \mathrm{D}\left(\beta_{T^{*} X}\left(\mathcal{E}_{X}^{\mathbf{R}, f}\right)\right)$ defined above over $\dot{T}^{*} X$ is sent to $\mu_{X} \mathcal{O}_{X}^{t}$ in $\mathrm{D}\left(\mathbf{I}\left(\mathbf{C}_{\dot{T}^{*} X}\right)\right)$ by the forgetful functor. Moreover, for $F \in \mathrm{D}^{-}\left(\mathbf{I}\left(\mathbf{C}_{X}\right)\right)$ the complex

$$
\alpha_{T^{*} X} \operatorname{RIH} \operatorname{Hom}\left(\pi^{-1} F, \mathcal{O}_{X}^{\mu}\right),
$$

which is naturally defined in $\mathrm{D}\left(\mathcal{E}_{X}^{\mathbf{R}, f}\right)$, over $\dot{T}^{*} X$, is isomorphic in $\mathrm{D}\left(\mathbf{C}_{\dot{T}^{*} X}\right)$ to $T$ - $\mu$ hom $\left(F, \mathcal{O}_{X}\right)$ endowed with its action of $\mathcal{E}_{X}^{\mathbf{R}, f}$.

\section{Acknowledgements}

The starting point of this paper is a discussion with Raphaël Rouquier and Pierre Schapira. The author also thanks Luca Prelli for his comments, especially about soft sheaves on the subanalytic site.

\section{References}

[1] E. Andronikof, Microlocalisation tempérée, Mém. Soc. Math. France (N.S.) 57 (1994). Zbl 0805.58059 MR 1273991

[2] J. Bernstein and V. Lunts, Equivariant sheaves and functors, Lecture Notes in Math. 1578, Springer, Berlin, 1994. Zbl 0808.14038 MR 1299527

[3] E. Bierstone and P. Milman, Semianalytic and subanalytic sets, Inst. Hautes Études Sci. Publ. Math. 67 (1988), 5-42. Zbl 0674.32002 MR 0972342

[4] M. Kashiwara, The Riemann-Hilbert problem for holonomic systems, Publ. Res. Inst. Math. Sci. 20 (1984), 319-365. Zbl 0566.32023 MR 0743382

[5] M. Kashiwara and P. Schapira, Sheaves on manifolds, Grundlehren Math. Wiss. 292, Springer, Berlin, 1990. Zbl 0709.18001 MR 1299726

[6] Moderate and formal cohomology associated with constructible sheaves, Mém. Soc. Math. France (N.S.) 64 (1996). Zbl 0881.58060 MR 1421293

[7] _ Ind-sheaves, Astérisque 271 (2001). Zbl 0993.32009 MR 1827714

[8] , Categories and sheaves, Grundlehren Math. Wiss. 332, Springer, Berlin, 2006 Zbl 1118.18001 MR 2182076

[9] M. Kashiwara, P. Schapira, F. Ivorra and I. Waschkies, Microlocalization of ind-sheaves, in Studies in Lie theory, Progr. Math. 243, Birkhäuser, 2006, 171-221. Zbl 1098.35008 MR 2214250 
[10] L. Prelli, Sheaves on subanalytic sites, Rend. Sem. Mat. Univ. Padova 120 (2008), 167-216. Zbl 1171.32002 MR 2492657

[11] Microlocalization of subanalytic sheaves, C. R. Math. Acad. Sci. Paris 345 (2007), 127-132. Zbl 1159.14034 MR 2344810

[12] M. Sato, T. Kawai and M. Kashiwara, Microfunctions and pseudo-differential equations, in Hyperfunctions and pseudo-differential equations (Katata, 1971), Lecture Notes in Math. 287, Springer, 1973, 265-529. Zbl 0277.46039 MR 0420735

[13] J.-C. Tougeron, Idéaux de fonctions différentiables, Ergeb. Math. Grenzgeb. 71, Springer, Berlin, 1972. Zbl 0251.58001 MR 0440598 